NASA Contractor Report 3004

\title{
Calculation of the Longitudinal Aerodynamic Characteristics of Upper-Surface-Blown Wing-Flap Configurations
}

Michael R. Mendenhall and Selden B. Spangler

Nielsen Engineering \& Research, Inc.

Mountain View, California

Prepared for

Langley Research Center

under Contract NAS1-14086

\section{N/SA}

National Aeronautics

and Space Administration

Scientific and Technical

Information Office

1978 


\section{Section}

$\begin{array}{lr}\text { INTRODUCTION } & 2\end{array}$

$\begin{array}{ll}\text { SYMBOLS } & 4\end{array}$

$\begin{array}{ll}\text { ANALYSIS } & 7\end{array}$

$\begin{array}{ll}\text { Wing-Flap Vortex-Lattice Model } & 7\end{array}$

$\begin{array}{ll}\text { General description } & 7\end{array}$

$\begin{array}{ll}\text { Aerodynamic forces } & 10\end{array}$

Vortex-Ring Jet-Wake Model 13

Quadrilateral jet model 13

Centerline specification $\quad 15$

Vortex-ring distribution $\quad 15$

$\begin{array}{ll}\text { Jet description } & 17\end{array}$

USB Interference Calculation Procedure 20

$\begin{array}{lrl}\text { RESULTS } & 21\end{array}$

Wing-Flap $\quad 22$

Jet Wake $\quad 23$

Single ring characteristics $\quad 23$

Jet characteristics $\quad 23$

Complete Configuration $\quad 25$

Corrections $\quad 25$

$\begin{array}{ll}\text { Data available. } & 27\end{array}$

$\begin{array}{lr}\text { Data Comparisons } & 28\end{array}$

$\begin{array}{ll}\text { CONCLUDING REMARKS } & 35\end{array}$

APPENDIX A - Induced Velocity Field Due to a Quadrilateral Vortex Ring $\quad 39$

REFERENCES

TABLES

\begin{tabular}{lc} 
FIGURES & 48 \\
\hline
\end{tabular} 


\section{CALCULATION OF THE LONGITUDINAL AERODYNAMIC CHARACTERISTICS OF UPPER-SURFACE-BLOWN \\ WING-FLAP CONFIGURATIONS}

$$
\text { by }
$$

Michael R. Mendenhall and Selden B. Spangler Nielsen Engineering \& Research, Inc.

\section{SUMMARY}

An investigation has been carried out to develop an engineering method for predicting the longitudinal aerodynamic characteristics of wing-flap configurations with upper surface blowing (USB). Two potential flow models were incorporated into the prediction method: a wing and flap lifting surface model and a jet wake model. The wing-flap model uses a vortex-lattice to represent the wing and flaps. The wing may have an arbitrary planform and camber and twist. The flap system may be made up of a coanda flap and other flap segments of arbitrary size. The jet wake model consists of a series of closely spaced rectangular vortex rings, which are positioned such that the wake is tangent to the upper surfaces of the wing and flap between the exhaust nozzle and the flap trailing edge. The wake model has a rectangular cross section over its entire length, and the wake can be specified such that the mass, momentum, and spreading rates are similar to actual usB jet wakes.

Comparisons of measured and predicted pressure distributions, spanload distributions on each lifting surface, and total lift and pitchingmoment coefficients on swept and unswept USB configurations are included in this report. A wide range of thrust coefficients and flap deflection angles is considered at angles of attack up to the onset of stall. Results indicate that overall lift and pitching-moment coefficients are predicted reasonably well at low thrust levels. The predicted detailed load distributions are qualitatively correct and the peak loads in the region of direct jet wake interference are in good agreement with experiment. 


\section{INTRODUCTION}

The short take-off and landing requirements for STOL aircraft necessitate a means of achieving very high lift coefficients on aircraft in take-off or landing configuration with little sacrifice in cruise performance. The upper surface blown (USB) flap provides such a means with the added benefit of reduced ground noise levels due to shielding of the engine and near wake by the wing. The jet efflux from turbofan engines mounted above the wing is allowed to impinge directly on the upper wing surface such that the jet wake becomes attached to the wing and flap surfaces, flows aft over the lifting surfaces, and is deflected by the trailing edge flap (figure 1). A large amount of additional lift is produced through engine wake deflection and jet-induced interference effects

Although numerous experimental investigations have been carried out to study the longitudinal aerodynamic characteristics of USB configurations, only a few analytical methods are available to predict these characteristics. The thin jet flap theory of reference 1 has been applied to USB configurations; however, the thin jet flap approach is not a realisti model for typical USB jets. This particular theory has been shown to underpredict the lift on USB configurations; for example, see reference 2 A linear inviscid theory to predict the aerodynamic interference between a wing and thick USB jet is presented in references 2 and 3 . This method has been successful in predicting the jet-induced incremental forces and moments on USB configurations even though the jet is assumed to be uniform in velocity and shape over its length and jet entrainment is neglected.

The purpose of this report is to describe an engineering method to predict the longitudinal aerodynamic characteristics of multiple jet USB configurations. The method utilizes potential flow models of the wing and flaps and the jet wake to carry out the interference calculations. The wing-flap lifting-surface model is a nonplanar, nonlinear, vortexlattice scheme. The jet wake model is a rectangular vortex ring distribution which is determined from empirical information on the flow in USB jets. The jet model is positioned a priori tangent to the wing and flap upper surfaces where it induces an interference velocity field on the lifting surfaces. An additional loading placed on the flap surfaces to account for the reaction force caused by jet deflection also induces a velocity field on the lifting surfaces. The tangency condition satisfied 
at the wing and flap control points results in a total loading which represents the aerodynamic loading, the jet reaction force, and an additional jet-induced loading which is often called supercirculation. The end result is the total wing-flap loading including usB jet interference, the distribution of loading, the total load on each individual lifting surface, and the distribution of loading on each surface. The method is also capable of computing the total induced velocity field at a field point off the lifting surfaces.

This report presents the technical approach to the problem, the development of the flow models, and comparisons with experimental data. A user's manual for the resulting computer program and companion report for the present report is contained in reference 4 . 


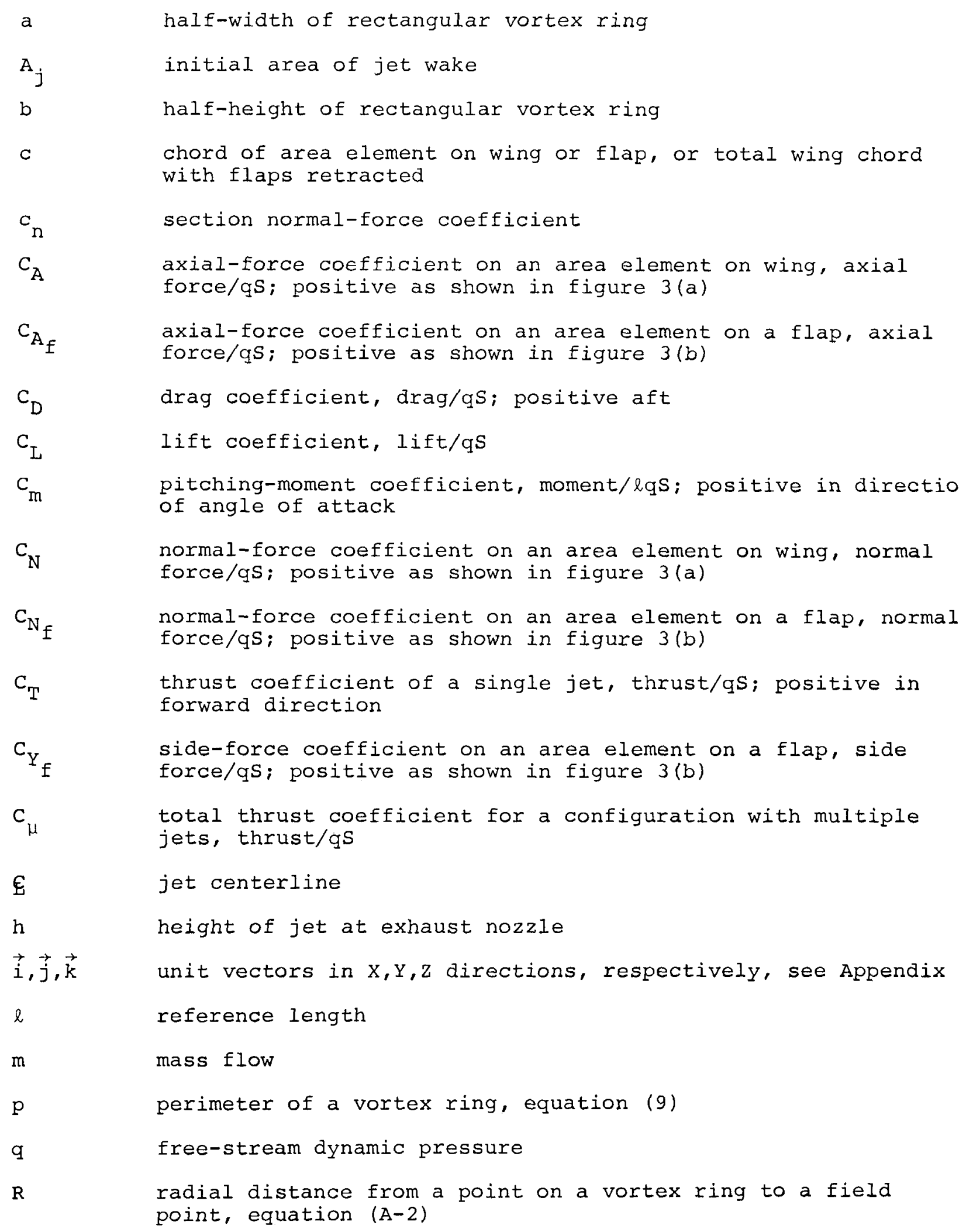




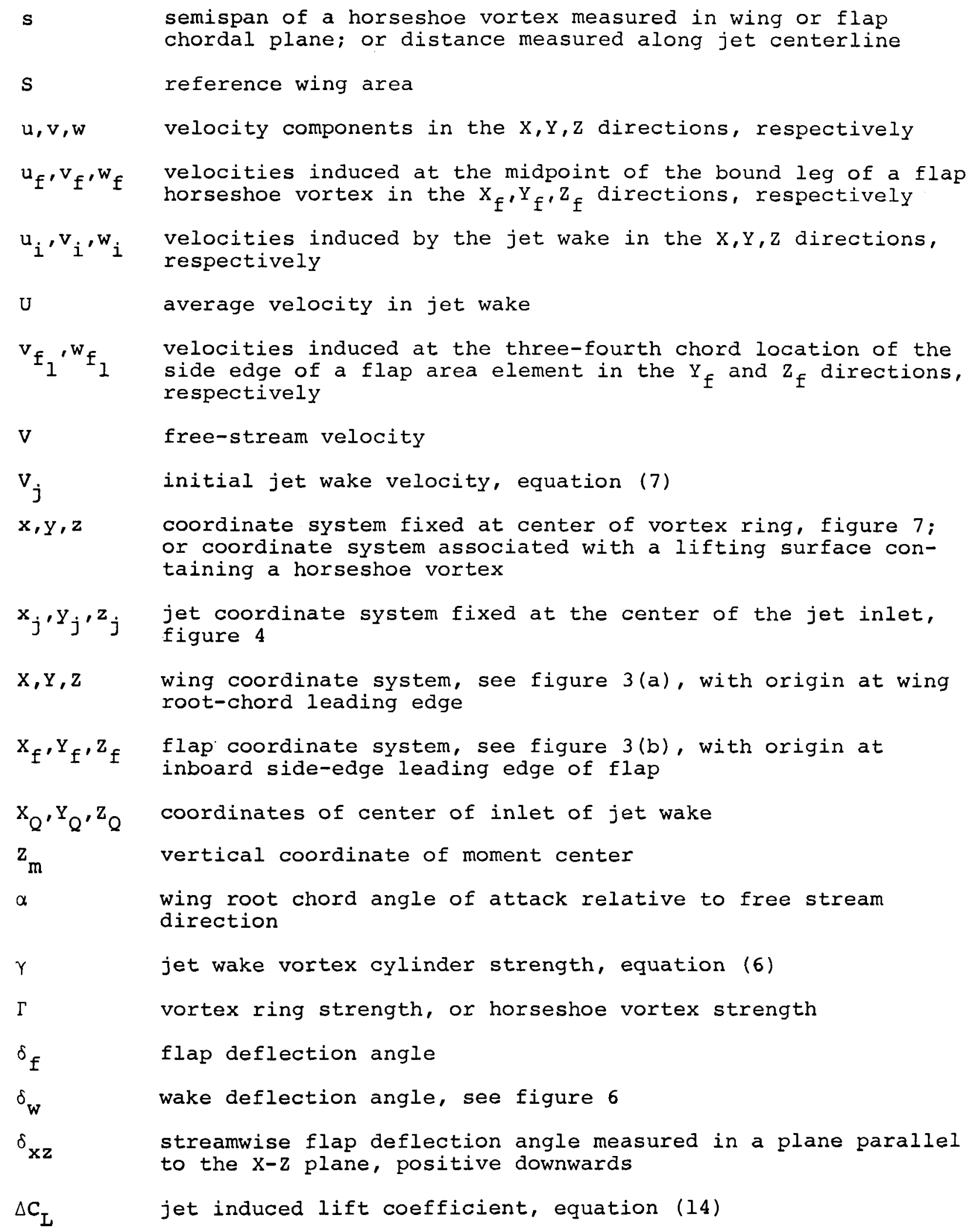

$s$

$S$

$u, v, w$

$u_{f}, v_{f}, w_{f}$

$u_{i}, v_{i}, w_{i}$

$\mathrm{U}$

$v_{f_{1}}, w_{f_{1}}$

$\mathrm{V}$

$v_{j}$

$x, y, z$

$x_{j}, y_{j}, z_{j}$ jet coordinate system fixed at the center of the jet inlet,

$X, Y, Z$ wing coordinate system, see figure 3(a), with origin at wing root-chord leading edge

$X_{f}, Y_{f}, Z_{f} \quad$ flap coordinate system, see figure $3(b)$, with origin at

inboard side-edge leading edge of flap

$\mathrm{X}_{Q}, \mathrm{Y}_{Q}, \mathrm{z}_{\mathrm{Q}}$

$\mathrm{z}_{\mathrm{m}}$

$\alpha$

$\gamma$

$\Gamma$

$\delta_{f}$

$\delta_{W}$

$\delta \mathbf{x z}$

$\Delta \mathrm{C}_{\mathrm{I}}$

semispan of a horseshoe vortex measured in wing or flap chordal plane; or distance measured along jet centerline

reference wing area

velocity components in the $X, Y, Z$ directions, respectively

velocities induced at the midpoint of the bound leg of a flap horseshoe vortex in the $\mathrm{X}_{f}, \mathrm{Y}_{\mathrm{f}}, \mathrm{Z}_{\mathrm{f}}$ directions, respectively

velocities induced by the jet wake in the $X, Y, Z$ directions, respectively

average velocity in jet wake

velocities induced at the three-fourth chord location of the side edge of a flap area element in the $Y_{f}$ and $Z_{f}$ directions, respectively

free-stream velocity

initial jet wake velocity, equation (7)

coordinate system fixed at center of vortex ring, figure 7; or coordinate system associated with a lifting surface containing a horseshoe vortex

coordinates of center of inlet of jet wake

vertical coordinate of moment center

wing root chord angle of attack relative to free stream direction

jet wake vortex cylinder strength, equation (6)

vortex ring strength, or horseshoe vortex strength

flap deflection angle

wake deflection angle, see figure 6

streamwise flap deflection angle measured in a plane parallel to the $x-Z$ plane, positive downwards

jet induced lift coefficient, equation (14) 


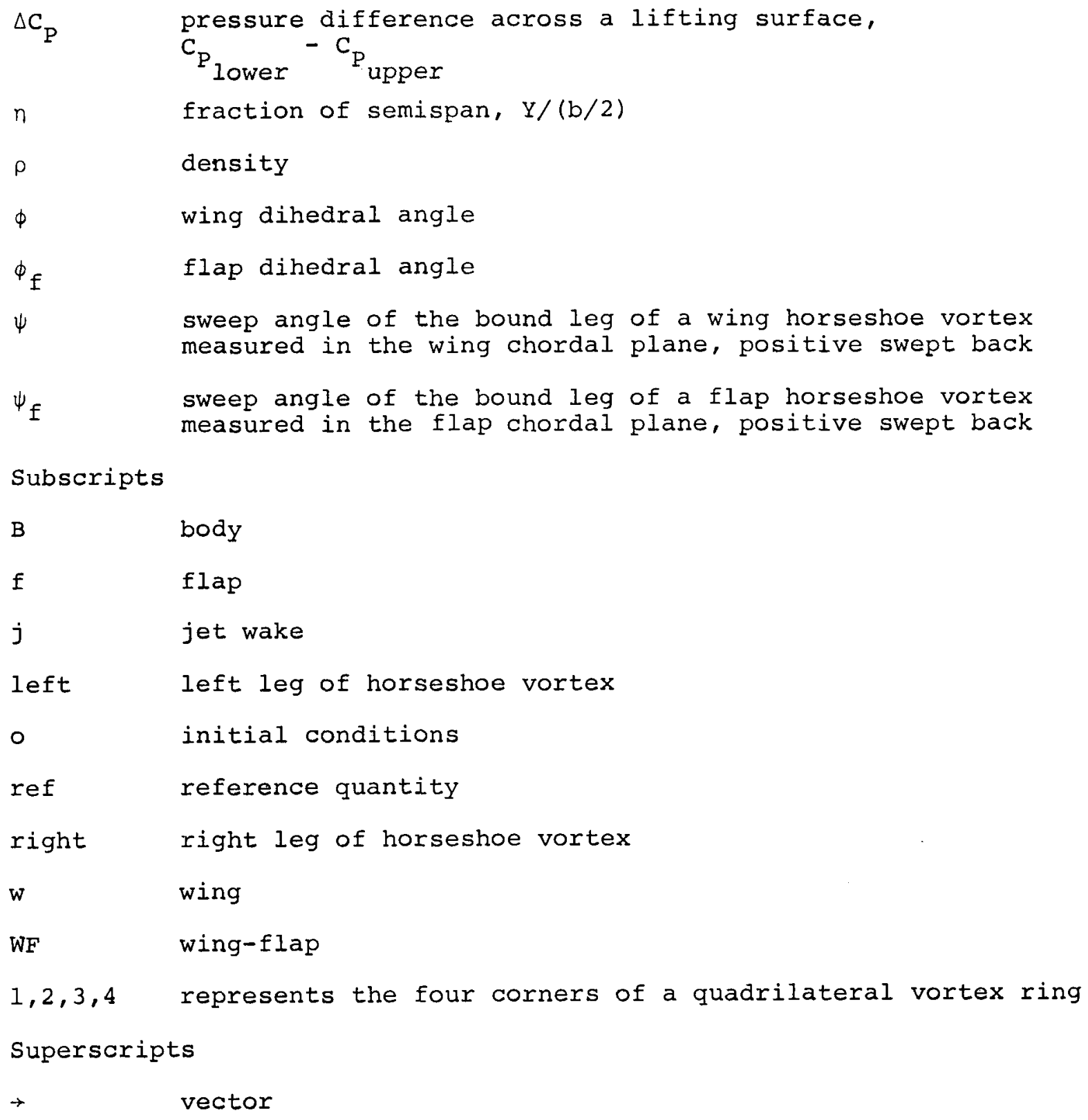




\section{ANALYSIS}

The work described in this report is an extension and improvement of that reported in reference 5. Improvements were made in both the lifting-surface model and the jet model, and both models were combined into a single program for the calculation of the aerodynamic characteristics of USB configurations. In this section, each flow model is discussed individually, and then the total USB interference procedure is described.

\section{Wing-Flap Vortex-Lattice Model}

General description. - The vortex-lattice lifting-surface model used in the present investigation is the same as that used in reference 6 . Multiple spanwise flap segments are considered, and separate loading distributions on individual flap elements are available. Typical uppersurface-blowing (USB) configurations have multiple spanwise flap segments, each of which may be made up of multiple flap elements. In the present investigation each flap element is defined as a separate lifting surface for the purpose of computing forces and moments. On USB configurations with Coanda flaps or radius flaps with continuous curvature, the flap surfaces are modeled as a series of straight flaps with no slots or gaps separating them.

The vortex lattice method is capable of modeling the following characteristics of the wing and flap:

\section{Wing Panels}

Leading-edge shape: May have up to 30 breaks in sweep. Trailing-edge shape: Same as for leading-edge.

Taper: Determined from leading-edge and trailing-edge shapes. Tip chord: Parallel to root chord.

Dihedral: Arbitrary but constant over the semispan.

Mean camber surface: May have both twist and camber.

Thickness: Neglected.

\section{Flap Panels}

Number: Up to 10 individual flap elements; maximum of 3 at any spanwise region.

Location: First flap in any spanwise region is at or near wing trailing-edge; gaps are permitted between wing and first flap and succeeding flaps. 
Leading-edge shape: Straight line which may be swept.

Trailing-edge shape: Same as for leading-edge.

Taper: Linear.

Root chord: In plane parallel to vertical plane containing wing root chord.

Tip chord: Parallel to root chord.

Span: Full or partial span.

Deflection: Each flap may have a different deflection angle.

Mean camber surface: Each flap may have both twist and camber.

Thickness: Neglected.

The method is restricted to angle of attack; that is, yaw effects are not included. Compressibility effects are not included since large flap deflections are only used at low speeds. Angle of attack, flap deflection angle, dihedral angle, and twist and camber angle are accounted for in the boundary condjtion by trigonometric functions since some of these angles may be large.

The vortex-lattice arrangement and the coordinate system for a swept wing with trailing-edge flaps deflected are shown in figure 2. The wing and flaps are divided into trapezoidal area elements. The spanwise distribution of area elements on the flaps must be the same as on the wing section ahead of the flap, which means that the flap side edges will lie along chordwise edges of the wing area elements.

A horseshoe vortex is placed in each area element such that the bound leg lies along the quarter chord of the area element and the trailing legs lie along the side edges of the element in the plane of the element. The trailing legs trail back in the plane of the area element until the leading edge of the next surface is encountered. They are then deflected so that they lie in the plane of that surface. This deflection is continued through the last surface. The trailing legs are then extended aft to infinity in the plane of the last surface.

The position of the horseshoe vortices trailing legs was investigatec extensively. In early wing-flap vortex lattice models (ref. 7), the trailing vorticity from the last surface was directed along a line which was approximately half way between the plane of the aft lifting surface and the free stream direction. A more recent approach (ref. 8) allows the trailing vorticity to be free, and its final location is solved for in an iterative manner. This approach was rejected for the current work 
because of the large amount of computer time required for an iterative solution. The approach taken in early USB calculations (ref. 5) placed the trailing vorticity in the plane of the originating panel. This produced adequate results, but the flow field was somewhat unrealistic as the trailing vorticity was distributed over the entire region behind the wing and flaps.

The current approach of extending the trailing legs aft to infinity in the plane of the last surface is a modification of the method of reference 7. Applying both methods to a simple rectangular wing with a single flap deflected $40^{\circ}$ produced the following results. At $\alpha=10^{\circ}$, placing the wake at an angle of $20^{\circ}$ with the wing produced a normal force coefficient which was approximately one-percent less than that obtained from the current method which has the wake at a $40^{\circ}$ angle with the wing. At $\alpha=-5^{\circ}$, the wake at $20^{\circ}$ resulted in a normal force coefficient approximately six-percent less than that obtained from the current method. Based on these results, and the added benefit that placing all the trailing legs in the plane of the last flap removes them from direct interaction with the USB jet model, the vortex lattice model of reference 6 was retained.

One additional modification in the horseshoe vortices trailing legs is required on a wing with partial span flaps. As described in reference 7, the problem area lies at a spanwise station where there is a break in the spanwise flap segments; for example, such a break is illustrated in figure 2 between the second and third chordwise rows and between the fourth and fifth chordwise rows. The current vortex lattice model requires the chordwise rows of panels on the wing and flaps to be aligned; therefore, it is not possible to use the vortex arrangement suggested in reference 7 as a tentative solution to the problem. An alternative vortex arrangement has proved to be acceptable. At a semispan station at which there is a junction of two flap segments, the wing trailing vorticity is removed from the edge of each flap and repositioned along a common line which does not lie in the plane of either of the flap segments. It would be desirable to place all the trailing vorticity along a line midway between the flap segments; however, this is not possible for adjacent flap segments with different length flap chords and deflection angles. A simple solution is to place all the wing trailing vorticity, at the semispan station of the flap junction, in the plane of the wing. This 
solution had very little effect on the total loads on the configuration, and it produced reasonable span load distributions.

The flow tangency boundary condition is applied at the midspan of the three-quarter chord line of each area element. This boundary condition determines the strength of the wing-flap horseshoe vortex distribution such that the total induced velocity field at the control point on the panel is tangent to the panel. The velocity at the control point is induced by all the horseshoe vortices making up the vortex lattice and the singularities associated with the jet model. The wing and flap boundary condition equations are presented in detail in reference 6 , and they will not be repeated here.

Aerodynamic forces. - Once the strengths of the horseshoe vortices $(\Gamma / V)$ have been determined by solving the set of simultaneous equations satisfying the boundary condition, the aerodynamic forces acting on each area element on the wing and flaps can be calculated. The traditional method for force calculation on vortex lattice panels is to apply the Kutta-Joukowski law for the aerodynamic force on a vortex filament. This method of force calculation is described in reference 6 . The components of force on the individual panels are presented as follows.

The axial-force coefficient produced by one of the area elements on the left wing panel is

$$
C_{A}=-\frac{2 \Gamma}{S V}(2 s)\left(\cos \phi \sin \alpha-\frac{W}{V} \cos \phi+\frac{V}{V} \sin \phi\right)
$$

Similarly, the normal-force coefficient is

$$
\begin{aligned}
C_{N}= & \frac{2 \Gamma}{S V}(2 s)\left(\frac{V}{V} \tan \psi+\cos \alpha \cos \phi-\frac{u}{V} \cos \phi\right) \\
& +\frac{\sum \Gamma}{S V}\left[\left(\frac{C v_{1}}{V}\right)_{\text {left }}-\left(\frac{C_{1}}{V}\right)_{\text {right }}\right]
\end{aligned}
$$

Positive directions of the forces are shown in figure $3(a)$. In the above equations, $2 s$ is the width of the panel in the wing chordal plane (the $z=0$ plane containing the root chord). The velocities $u, v$, and $w$, positive in the positive $X, Y, Z$ directions, are those induced at the bound-leg midpoint by the entire wing-flap vortex lattice (and wake, if present). The angle $\psi$ is the sweep angle of the bound leg, positive if swept back. 
The second term in equation (2) is the net force on all of the vortex trailing legs lying on the two side edges of the panel. The vortices are only those lying in the chordwise strip containing the panel. In equation (2), $\Sigma \Gamma$ is the sum of the strengths of these vortices, $c$ is the length of the panel side edge, and $v_{j}$ is the vortex lattice induced velocity at the three-quarter chord location of the side edge. The subscripts left and right refer to the left side edge and right side edge of the panel.

Similar expressions can be written for the forces acting on a panel on a flap. Positive directions of the forces are shown in figure $3(b)$. The axial-force coefficient is,

$$
C_{A_{f}}=-\frac{2 \Gamma}{S V}(2 s)\left[\cos \phi_{f} \cos \left(\alpha+\delta_{x z}\right)-\frac{w_{f}}{V} \cos \phi_{f}+\frac{v_{f}}{V} \sin \phi_{f}\right]
$$

the side-force coefficient is,

$$
\begin{aligned}
c_{Y_{f}}= & \frac{2 \Gamma}{S V}(2 s)\left[\cos \left(\alpha+\delta_{x z}\right) \sin \phi_{f}-\frac{u_{f}}{V} \sin \phi_{f}-\sin \left(\alpha+\delta_{x z}\right) \tan \psi_{f}\right. \\
& \left.+\frac{w_{f}}{V} \tan \psi_{f}\right]+\frac{\sum \Gamma}{S V}\left[\left(c \frac{w_{f}}{V}\right)_{\text {left }}-\left(c \frac{w_{f}}{V}\right)_{\text {right }}\right]
\end{aligned}
$$

and the normal-force coefficient is,

$$
\begin{aligned}
C_{N_{f}}= & \frac{2 \Gamma}{S V}(2 s)\left[\frac{v_{f}}{V} \tan \psi_{f}+\cos \left(\alpha+\delta_{x z}\right) \cos \phi_{f}-\frac{u_{f}}{V} \cos \phi_{f}\right] \\
& +\frac{\sum \Gamma}{S V}\left[\left(c \frac{v_{f_{I}}}{V}\right)_{\text {Ieft }}-\left(c \frac{v_{f}}{V}\right)_{\text {right }}\right]
\end{aligned}
$$

The velocities $u_{f}, v_{f}, w_{f}$ are positive in the positive $x_{f}, Y_{f}, z_{f}$ directions, respectively.

The elemental panel forces given by equations (1) through (5) can be used to calculate spanwise load distributions by summing over the area elements in a spanwise row, and total forces can be determined by then summing spanwise. Moments can be determined by using the panel forces with the appropriate moment arm.

For power-off calculations, equations (1) through (5) are used exactly as written, except for the edges of flaps. As discussed in the previous section, the wing trailing vorticity is removed from the edges of partial span flaps; consequently, this vorticity cannot contribute to 
the force on the panel edges. The calculation is handled by simply removing the $\Sigma \Gamma$ term in equations (4) and (5) when the panel under consideration lies at either the root or the tip of a partial span flap. This particular modification has very little effect on the predicted total forces and moments. The effect on the flap span load distributions is a smoothing of the distribution in the vicinity of the flap edges.

For power-on calculations, the panel forces are obtained from equations (1) through (5) as discussed above, but certain new interpretations are required. The horseshoe vortex strength, $\Gamma$, on a panel can be made up of four components. The first is a circulation due to the free stream flow over the wing-flap. The second component is a circulation which represents the jet reaction force on the flaps due to the deflection of the jet by the coanda effect. The third component is due to the flow field induced by the jet singularity distribution. The fourth component of the circulation on a panel is that induced by the loading associated with the jet reaction force. The latter two components represent the loading often called supercirculation. The net result is that the total circulation on a panel is much larger for power-on calculations than for power-off calculations. It follows that the perturbation velocities induced by the total circulation are larger. This can lead to difficulties in the calculation of forces using equations (1) through (5) because the large perturbation velocities acting on strong vortex filaments can produce inordinately large forces. The following modified approach is taken for power-on force calculations.

As was done in reference 2 , it is assumed that the jet wake lies a small distance above the wing and flap surfaces such that the high jet velocity does not act at the surface itself. Thus, the velocity acting on the bound circulation on the wing and flap is made up of the free stream velocity, perturbation velocities from the jet singularity model, and self-induced velocities from the circulation on the wing and flaps. The difficulty occurs when the perturbation velocities become large; for example, when they become the order of the free stream velocity. Results of extensive comparisons of measured and predicted span load distributions indicated that best results are obtained when the free stream velocity alone is used in the force calculations. This is the method used to obtain all power-on results presented in this report. 
A potential flow model of the wake from a turbofan engine is described in reference 9 . This model has a vorticity distribution placed on the specified boundary of an expanding circular or elliptic crosssection jet. The surface vorticity distribution is represented by a series of vortex rings coaxial with the wake centerline, each ring representing a finite increment of length of the cylinder. The strength of each ring is equal to the net vorticity on the incremental length of cylinder which it represents. By appropriate specification of the spreading of the wake, this vortex ring model represents the mass, momentum, and entrainment characteristics of a turbulent, coflowing jet. The vortex ring model produces realistic induced velocities outside the jet boundary because of the proper representation of the jet parameters, but velocity within the jet boundary is nearly uniform with this type of singularity distribution.

The influence of the lifting surface on the jet model is twofold. First, the jet wake is positioned such that its lower surface lies close to the upper surface of the wing and flap. This positioning determines the centerline location of the jet and the region throughout which the high jet velocity occurs. Secondly, the jet velocity is known to decay along the jet length due to jet expansion and mixing. Through the use of empirical jet data, the influence of the wing and flap surfaces are considered in specifying the velocity decay characteristics.

The major difference between the EBF wakes considered in reference 9 and typical USB wakes considered herein is the cross-sectional shape. Most USB models have rectangular or near-rectangular exhaust nozzles located on the wing upper surface. The following paragraphs will describe the development of a rectangular jet model and its use for usB configuration interference calculations.

Quadrilateral jet model. - The development of the rectangular crosssection potential flow jet model proceeds in similar fashion to the development of the circular jet model in references 9 and 10. The assumption is made here that the jet has a rectangular cross section over its entire length.

Consider for the moment that the jet vortex model is a constant radius, semi-infinite vortex cylinder with strength $\gamma / V$. The strength 
of this vortex cylinder is specified by the relation

$$
\frac{\gamma}{V}=\frac{v_{j}}{V}-1
$$

where $V_{j}$ is a uniform velocity across the engine exhaust exit. This velocity is given approximately as

$$
\frac{v_{j}}{V}=\frac{1}{2}\left[1+\sqrt{1+2 C_{T} \frac{S p}{A_{j}{ }^{\rho} j}}\right]
$$

where $C_{T}$ is the thrust coefficient for a single jet and $A_{j}$ is the exhaust exit area for a single jet. Equations (6) and (7) apply to a jet with any cross-sectional shape.

starting with a vortex cylinder with constant rectangular crosssection and strength $\mathrm{Y} / \mathrm{V}$, the actual jet wake is modeled by distorting the cylinder to fit a prescribed centerline path and outer boundary. The continuous vorticity distribution on the new distorted cylinder is replaced by a closely spaced series of rectangular vortex rings coaxial with the jet centerline. Each ring represents a small increment of lengtr. of the jet, and each ring has a shape which corresponds to the specified jet boundary. The strength of the ring vorticity is equal to the net vorticity on the incremental length of the original cylinder. Thus, the semi-infinite length of the original cylinder is replaced with a finite length of vortex rings. As described in reference 10, the semi-infinite length cylinder can be approximated to any required accuracy in this manner.

The strength of any vortex ring along the jet is

$$
\frac{\Gamma}{\mathrm{V}}=\frac{\gamma}{\mathrm{V}} \frac{\mathrm{P}_{\mathrm{O}}}{\mathrm{P}} \Delta \mathrm{s}
$$

where $P$ is the perimeter of the ring in question and $\Delta s$ is the length of the wake represented by the ring. For a rectangular cross section, the perimeter is

$$
P=4(a+b)
$$

where $\mathrm{a}$ and $\mathrm{b}$ are the semi-major and semi-minor axes of the rectangle.

From equation (8), the strength of the vortex ring decreases as its perimeter increases such that the product of $\Gamma$ and the ring perimeter is constant for equally spaced rings ( $\Delta \mathbf{s}=$ constant). This is equivalent to keeping the total vorticity on the cylinder constant. The induced 
velocity at any point in the field is calculated by summing the contributions from all the rings making up the model. The induced velocity field due to a single quadrilateral ring is calculated from the equations presented in reference 11. These results are reproduced in Appendix A.

Centerline specification. - In the EBF calculation of reference 9, the jet wake centerline could not be specified a priori; it could be located only through an iterative procedure. In the UsB flow model, the centerline can be positioned with respect to the wing and flaps with the assumption that the wake is attached to the wing and flap upper surfaces over the entire run length from the nozzle exit to the flap trailing edge. The user must specify the vertical and lateral expansion of the wake at various points along its length. This information, plus the requirement that the wake be raised up off of the wing and flap surfaces a small amount to insure that the lifting surfaces are in the wake outer flow field, is adequate to completely specify the position of the wake. A typical section through the centerline of the jet of a USB configuration is shown in figure 4 .

The centerline is assumed to be straight for a short distance behind the trailing edge of the last flap, and then it is allowed to return to its original direction via a parabolic arc. The induced effects of the jet wake are not strongly dependent on the wake position aft of the trailing edge; therefore, this simplified approach to its position is justified. Another advantage to this approach is that the jet model is a function only of the geometry of the configuration. Thus, the jet model does not require recalculation at each angle of attack. This results in a considerable savings in computer execution time at little expense in the accuracy of the results.

Vortex-ring distribution. - The quadrilateral vortex rings are distributed along the centerline in such a fashion that they form a lower boundary of the jet which is aprallel to the wing and flap surfaces. In the interest of obtaining smooth induced velocity distributions on the lifting surfaces, the spacing of the rings is such that each $r$ ing is normal to the adjacent lifting surface, and the spacing between the rings is equal at the center of the lower jet boundary. This results in a series of rings that are not necessarily normal to the centerline of the jet wake nor is the spacing along the centerline constant over the length of the jet, because the jet must bend through a large angle in passing 
over the Coanda flap. This approach is used to produce the best induced velocity field adjacent to the lower jet surface on the wing and flap.

In computing the induced velocity field associated with a series of quadrilateral vortex rings using the equations of Appendix $A$, it is important that the continuity of each ring in the jet model be maintained. If all the rings were normal to the $\mathrm{x}-\mathrm{z}$ plane or the plane of the centerline, a difficulty would arise when a ring intersects a swept wing or flap trailing edge. The lower side of the ring could be parallel to one or the other lifting surface, but it could not be parallel to both at the same time without breaking or segmenting the ring. This situation, illustrated in sketch 1 , is very difficult to set up for a general configuration, and when the proper orientation of the rings is achieved,

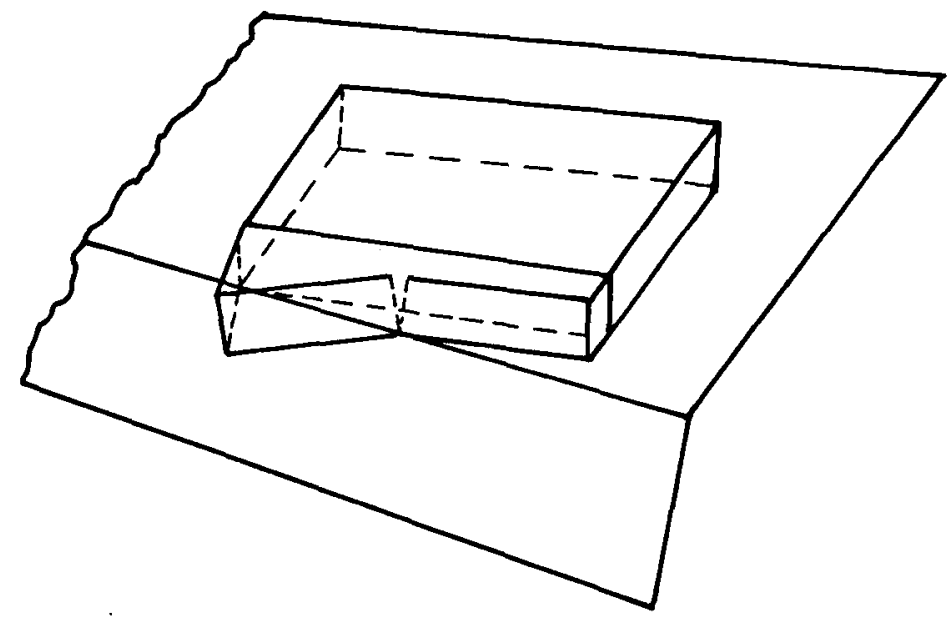

SKETCH 1.- SEGMENTED VORTEX RING MODEL

there are numerical problems associated with calculation of the induced velocity field.

An alternate approach involving skewed vortex rings has been successful in producing a jet model which has its lower surface parallel to the adjacent lifting surface. This model, illustrated in sketch 2, can be set up for general USB configurations, and it has exhibited no difficulties in the calculation of the induced velocity field.

The quadrilateral vortex rings are equally spaced along the centerline of the lower jet boundary from the jet inlet to the trailing edge of 


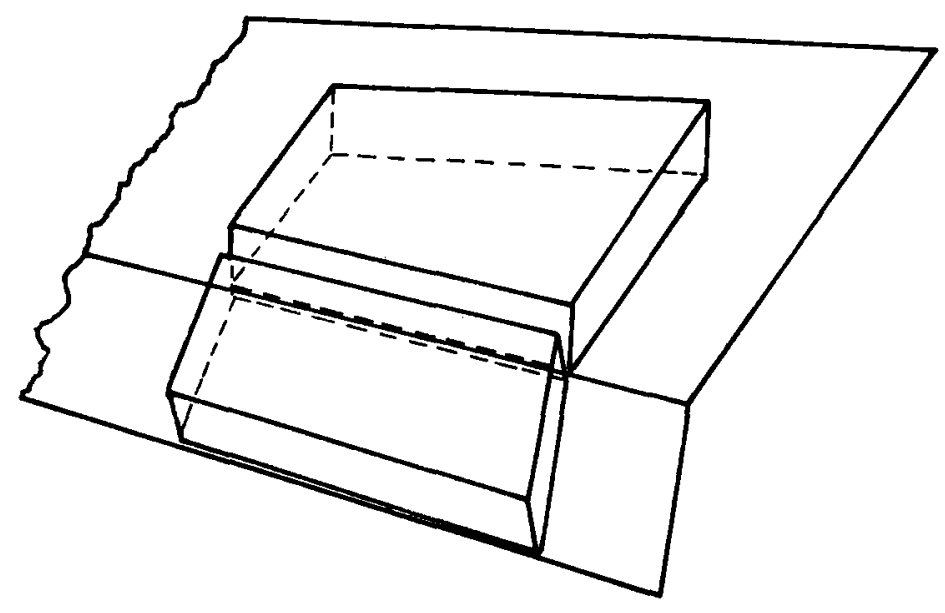

SKETCH 2.- SKEWED VORTEX RING MODEL

the last flap. The spacing is also equal on the inboard and outboard sides of the jet, but the rings are skewed so that spacing is not the same on both sides, nor is it the same as the specified spacing on the centerline. There is a small effect of the skewed rings on the induced velocity field on the wing and flap surfaces. The induced $u$ - and w-velocities are nearly the same as previous straight ring results. The skewed rings induced a small v-velocity at the center of the jet but this has no effect on the wing boundary condition if there is no dihedral. For wings with small dihedral angles, the v-velocities near the jet centerline have a small effect on the final wing and flap loading. This skewed vortex ring orientation is used for all results presented in this report.

Jet description. - The rectangular jet model requires three items to determine the analytical description of the jet. The first two items are the jet thrust coefficient, $C_{T}$, and the density ratio, $\rho / \rho_{j}$, which are used in equation (7) to specify the initial jet velocity at the exhaust nozzle. The remaining item is the jet boundary which is determined by the lengths of the semi-major and semi-minor axes at various points along the length of the wake. The computer program described in reference 4 automatically locates the jet wake in the proper position with respect to the wing and flaps. The jet turning efficiency of the configuration is an optional item in the specifications of the jet model. This is an empirically determined parameter which is the ratio of the actual deflection of the jet to the maximum flap angle. This parameter is used to 
limit the amount of the jet momentum transferred to the flaps through the deflection of the jet wake. For moderate flap angles $\left(0<\delta_{f}<30^{\circ}\right)$ the turning efficiency is typically between 0.9 and 1.0 , but for large deflection angles $\left(45^{\circ}<\delta_{f}<90^{\circ}\right)$ the turning efficiency can drop to 0.8 or lower.

The boundary of the jet must be specified at all points along the length of the jet. Since the character of the jet-induced velocity field depends on the shape and expansion of the jet model, some care should be taken to insure that the jet model represents the actual jet. Specification of the axes of the rectangular cross sections allows some freedom in matching the lateral and vertical expansion characteristics of typical USB jet wakes. Each axis may be specified, independent of the other, over the entire length of the jet; unfortunately, very little information is available to determine the proper vertical and lateral expansion rates of rectangular USB jets. A crude estimate of the lateral expansion of a typical USB jet can be made from observations of soot deposits on the Coanda flap of the aircraft model in reference 12. This configuration, similar to the model used in reference 13, appears to have only a small amount of lateral expansion. Similar unpublished photographs of the upper surface of the two-engine USB model of reference 14 indicate that the jet expands laterally a sufficient amount to cover nearly the entire width of the Coanda flap at its trailing edge. None of this information has enough detail to permit any quantitative conclusions regarding lateral expansion of USB jets.

Information on the vertical expansion of USB jets is available in reference 15. In this reference, velocity profiles on the centerline of the inboard engine are measured on the upper wing and flap surfaces for a wide range of engine thrust coefficients. These data, taken at one semispan station only, indicate much about the vertical expansion and the velocity decay of USB jets between the exhaust nozzle and a point on the coanda flap. In a distance of eight nozzle heights downstream, the total jet height grows approximately 508. It is difficult to determine the jet height exactly from these data, but it appears that the vertical expansion of the jet downstream of the nozzle is nearly independent of the jet momentum or thrust coefficient.

The decay of the jet velocity downstream of the nozzle was determined by integrating the measured velocity profiles in reference 15 to find the 
average velocity at each downstream station. These average velocities, normalized by the velocity at the exhaust nozzle, are shown in figure 5 for three different flow conditions. The results indicate a general decay of the jet velocity, but there is too much scatter in the data to isolate the effect of thrust coefficient. Neglecting any effect of thrust coefficient, each of the three sets of data in figure 5 was fit with a linear curve using a least squares approach. These curves are shown dashed in the figure.

The information just described is used to specify the boundary of the jet in the following manner. The data on lateral and vertical spreading of the jet is too limited to use directly in the specification of the boundary; however, the velocity decay data in figure 5, coupled with a property of vortex ring jet models, can be used to specify the required jet boundary. It is a property of a rectangular vortex ring jet model that the average velocity in the jet wake at any puint is related to the perimeter of the jet at that point by the relation

$$
\frac{\mathrm{U}}{\mathrm{U}_{\mathrm{O}}}=\frac{\mathrm{P}_{\mathrm{O}}}{\mathrm{P}}
$$

The initial perimeter of the jet, $P_{O}$ ' is specified by the exhaust nozzle, and the velocity ratio is read from the curves in figure 5 . Substituting equation (9) into (10) results in an expression for the semi-major and semi-minor axes of the jet at a point. One additional plece of information is required to relate the length of the two axes. At. this point, we can assume that either the vertical or lateral spraading is known and calculate the other axis length. An alternate approich is to assume that the aspect ratio of the jet is constant for the portion of the jet attached to the upper wing and flap surfares. This latter approach is the most reliable unless some specific information regarding jet spreading is available. No matter which approach is taken, the user should examine the final width of the jet at the flap trailing edge to be sure that the jet does not extend too far beyond the tip of the flap. It has been observed on typical USB models that the jet generally does not expand to a width greater than the span of the coanda flap at its trailing edge.

It should be emphasized here that several assumptions have been made regarding the velocity profiles from reference 15 used to generate the curves of figure 5. Out of necessity añd lack of data, it is assumed 
that thin velocity profiles are similar across the jet width at all station:s downstream of the exhaust nozzle. This is not an unreasonable assumption for the jet at the exhaust nozzle as illustrated in reference 12. The authors did not have access to any published information on velocity profile measurements across the width of a jet at stations downstream of the nozzle. Use of the data of reference 15 to produce the curves in figure 5, which in turn are used to model general usB jets, presumes that there is no influence of geometry on the decay of USB jet wakes. This is very likely not the case, but in the absence of additional data to supplement reference 15, we must assume that we can use figure 5 for all configurations.

USB Interference Calculation Procedure

Calculation of the aerodynamic loading of a wing-flap configuration under the influerce of the jet wake from an upper surface mounted turbofan engine is done wi=h the combination of the two potential flow models described in the rreceding sections. The two flow models are combined by superposition. The jet model induces a velocity field on the wing and flaps which produces an interference loading on the lifting surfaces. An additional lcading occurs on the wing and flaps due to the deflection of the jet by the flaps. It is assumed that the engine thrust is unaffected by the presence of the wing and flap surfaces, and it is also assumed that the jet remains attached to the upper surfaces of the wing and flaps. The sclution is carried out in the following manner.

The jet wake poition is known initially, because the jet is assumed to be attached to the upper surface of the wing and flap. We also assume that the wing is entirely in the outer flow field of the jet; therefore, in actual practice the jet wake is positioned slightly above the actual lifting surface of the wing and flaps. The jet model induced velocity field is computed at the control points on the lifting surfaces by summing the effects of the individual vortex rings.

Since the jet wake is located a priori tangent to the wing and flap surfaces, some means is needed to represent the force required to deflect the jet over the flap. We know that the downward force required to deflect the jet wake, $C_{T} \sin \delta_{f}$ is equal in magnitude and opposite in direction to the reaction force or the wing and flaps. Thus, what is needed is a means to represent tw; force on the lifting surfaces. The 
average velocity at each downstream station. These average velocities, normalized by the velocity at the exhaust nozzle, are shown in figure 5 for three different flow conditions. The results indicate a general decay of the jet velocity, but there is too much scatter in the data to isolate the effect of thrust coefficient. Neglecting any effect of thrust coefficient, each of the three sets of data in figure 5 was fit with a linear curve using a least squares approach. These curves are shown dashed in the figure.

The information just described is used to specify the boundary of the jet in the following manner. The data on lateral and vertical spreading of the jet is too 1 imited to use directly in the specification of the boundary; however, the velocity decay data in figure 5, coupled with a property of vortex ring jet models, can be used to specify the required jet boundary. It is a property of a rectangular vortex ring jet model that the average velocity in the jet wake at any puint is related to the perimeter of the jet at that point by the relation

$$
\frac{\mathrm{U}}{\mathrm{U}_{\mathrm{O}}}=\frac{\mathrm{P}_{\mathrm{O}}}{\mathrm{P}}
$$

The initial perimeter of the jet, $P_{O^{\prime}}$ is specified by the exhaust nozzle, and the velocity ratio is read from the curves in figure 5. Substituting equation (9) into (10) results in an expression for the semi-major and semi-minor axes of the jet at a point. One additional piece of information is required to relate the length of the two axes. At this point, we can assume that either the vertical or lateral spraading is known and calculate the other axis length. An alternate approach is to assume that the aspect ratio of the jet is constant for the portion of the jet attached to the upper wing and flap surfares. This latter approach is the most reliable unless some specific information regaraing jet spreading is available. No matter which approach is taken, the user should examine the final width of the jet at the flap trailing edge to be sure that the jet does not extend too far beyond the tip of the flap. It has been observed on typical USB models that the jet generally does not expand to a width greater than the span of the coanda flap at its trailing edge.

It should be emphasized here that several assumptions have been made regarding the velocity profiles from refarence 15 used to generate the curves of figure 5. Out of necessity änd lack of data, it is assumed 
total deflection force is distributed over all the panels which lie directly beneath the jet in such a manner that the force per unit area on all panels on a given flap is constant. A circulation is placed on each panel such that the circulation, when acted on by the free stream velocity, produces a force exactly equal to the panel force associated with the jet deflection. This circulation is considered the additional loading associated with the deflection of the jet wake, and it in turn induces a velocity field at all other control points on the wing and flaps.

Now having the total jet induced velocity at each control point, the circulation distribution on the wing and flaps is obtained such that the tangency boundary condition is satisfied at each control point. On each panel, the total circulation is made up of four components described previously. The first component is the aerodynamic circulation satisfying the panel boundary condition, and the second component is the additional circulation associated with the force on the panel due to the turning of the jet wake. The third component is due to the induced flow field from the jet singularity distribution, and the last component is the circulation on the wing and flaps due to the induced velocity field of the additional circulation associated with the jet reaction force. Thus, the total wing and flap loading is made up of an aerodynamic component of circulation which is independent of the jet, and three components representing the interaction force of the jet on the lifting surfaces.

\section{RESULTS}

The methods of analysis described in the previous section are evaluated in the following manner. Each flow model (the lifting surface and the jet wake) is examined separately to determine its individual characteristics. Where possible, comparisons with data are made to assist in the evaluation. After the individual flow models are examined, they are combined to perform a number of calculations on usB configurations. Comparisons with experimental data are used to evaluate the total USB prediction method. The results and data comparisons are discussed below. 


\section{Wing-Flap}

The vortex lattice method used in the current usB prediction method was applied to many different wing-flap configurations. The results showed that the vortex lattice method worked well in predicting the longitudinal aerodynamic characteristics of both swept and unswept wings with multiple flaps. The effect of deflecting the trailing vorticity at various angles with respect to the flaps is described as follows.

The configuration used to check out the effect of the angle of the deflected wake is a thin cylindrical fuselage with an aspect ratio 6 rectangular wing and a single slotted flap (ref. 16). The Fowler-type flap has a chord that is 30 percent of the wing-alone chord and is deflected $40^{\circ}$. The vortex-lattice arrangement used for these calculations is 8 chordwise by 10 spanwise on the wing semispan and 4 by 10 on the flap. The trailing legs of all the horseshoe vortices are deflected at angles of $0^{\circ}, 20^{\circ}, 40^{\circ}$, and $50^{\circ}$ with respect to the plane of the wing. A sketch defining the wake deflection angle is shown in figure 6 .

Comparison of measured and predicted lift coefficients on this model are presented in figure 6 where a systematic effect of the wake angle car. be seen. At low angles of attack, the lift coefficient increases with increasing wake deflection angle. At high angles of attack, the wake induced effects are small when compared with the free stream induced effects; therefore, there is an almost negligible effect of wake angle on the predicted lift coefficient. There is a large effect of the wake angle on the lift curve slope. Note that the effect of the fuselage is not included in the predicted results. The inclusion of the fuselage should have the effect of increasing the lift coefficient as a function of the angle of attack, thus increasing the lift curve slope.

Referring to figure 4 , we can see that an attached jet leaving the trailing edge of the last flap tangent to that flap will likely have the effect of forcing the trailing vorticity to follow along. Downstream of the trailing edge, when the jet starts bending in the direction of the free stream, the trailing vorticity will also move in that direction. That point is sufficiently downstream of the lifting surfaces that the position of the trailing vorticity has very little effect on the induced flow field near the wing and flaps. Based on this reasoning, and the results of figure 6 , the trailing vorticity is allowed to deflect with the flap system and trail to infinity in the plane of the last flap. In 
the particular application of the vortex lattice method used in this report, deflection of the vortex wake at the flap angle also has the advantage of removing it from direct interaction with the jet wake model. All further results presented in this report will be calculated with the wake deflection angle equal to the flap deflection angle.

Jet Wake

Single ring characteristics. - Since the complete jet model is made up of a series of vortex rings, it is appropriate that the characteristics of a single, rectangular vortex ring (figure 7) be examined. The induced velocity field due to a circular vortex ring is documented in reference 17, and similar documentation for an elliptic vortex ring is presented in reference 9. The induced velocity field near a rectangular vortex ring is easily calculated using the analysis presented in the appendix. Some induced velocities at various points near single rectangular vortex rings of different axis ratios are shown in Tables I and II. The general characteristics of the induced velocities associated with rectangular rings are similar to those described for elliptic rings in reference 9 , and at some points in the flow field there is very little difference in the induced velocities.

There is the usual singularity in the plane of the ring at the edge of the ring, but this causes no computational difficulties since this region of the vortex ring is never used when computing the induced velocity field due to the entire jet wake. Field points which fall between two adjacent rings are treated as if they lie exactly half way between the two adjacent rings. This correction is carried out to achieve smooth induced velocity profiles which are in agreement with the profiles which would be obtained from a uniform distribution of vorticity on a semiinfinite cylinder.

Jet characteristics. - The jet model is made up of a series of coaxial vortex rings of rectangular shape, and the induced flow field at any point is determined by adding the contribution of each ring in the jet model. Even though the induced velocity from a single ring is highly nonuniform, as shown in Tables I and II, the total induced velocity field associated with the complete jet is relatively smooth. The jet-induced axial velocity profiles at various points inside circular and elliptic jets, discussed in reference 9, exhibit uniform velocity inside the 
boundary of the jet and nearly zero velocity outside the boundary. As would be expected, exactly the same characteristics are observed for rectangular cross section jets.

As the jet expands with distance along the centerline, the induced axial velocity on the centerline decreases. The axial velocity is inversely proportional to the local perimeter of the jet as illustrated in figure 8. A number of jets, ranging from a square cross section to a 10:1 rectangular cross section, are shown on this correlation curve. Various expansion rates are represented by the points in this figure, from nonexpanding to very large expansion rates. The correlation shown on figure 8 is completely independent of the cross sectional shape of the jet. The cross sections may be similar over the length of the jet or one axis may expand at a much higher rate than the other axis.

It can be shown that the mass inside the boundary of an expanding rectangular vortex ring jet model is not constant but is increasing with distance along the jet as long as the perimeter of the jet is increasing. This is illustrated in figure 9, which shows the ratio of the local mass flow to the initial mass flow in the jet as a function of the perimeter ratio. The curve in figure 9 applies only to jets which maintain a similar cross section (aspect ratio $a / b=$ constant) over their entire length. If the aspect ratio of the cross section changes with position along the jet, the mass ratio curve in figure 9 changes.

An investigation of the momentum inside the jet boundary shows it to remain nearly constant over the total length of the jet if the jet cross sections maintain a constant aspect ratio. If the jet cross sections vary in aspect ratio, the momentum is not constant and can change with the amount of jet expansion. For the typical USB jets to be considered with this jet model, the aspect ratio of the cross section changes so little and the expansion rate is so small that, for all practical purposes, the momentum can be considered constant over the full length of the jet wake.

Using the curves in figures 5 and 8 , a rectangular vortex ring jet model was designed to represent the aspect ratio 6 nozzle and jet wake of reference 15. Measured jet velocity profiles at several stations downstream of the exhaust nozzle are available for a wide range of thrust coefficients. The faired curve of figure 5 was used to specify the jet velocity at the trailing edge of the coanda flap, thus specifying the 
perimeter at that point. The jet boundary was allowed to expand linearly between the exhaust nozzle and the flap trailing edge. Measured and predicted axial velocity profiles on the centerline of the inboard engine are compared in figure 10 for four thrust coefficients. The predicted velocity profiles in this figure include the effect of the wing and flap loading on the configuration for a deflection of the Coanda flap of $60^{\circ}$. A sketch of the configuration, showing the chordwise locations of the velocity measurements, is shown in figure 10 .

The results in figure 10 show good agreement between measured and predicted velocity profiles at the station nearest the exhaust nozzle. It appears that the assumed linear expansion produces a thicker jet model at this station than is indicated by the data. The same situation occurs at the downstream station where the agreement between measured and predicted velocity profiles is not as good as is the upstream agreement. The maximum jet velocity near the flap surface is in good agreement, but the predicted average velocity at this station is too high. This is caused by the induced velocity from the loading on the wing and flaps.

\section{Complete Configuration}

The overall USB prediction method was evaluated by comparing predicted results with data on several typical USB configurations. Ideally, one would like to have wing-flap-engine data on overall forces and moments, loads on individual lifting segments, pressure distributions, and jet wake velocity surveys. A large amount of data is available, but most of it is on complete configurations and consists only of overall force and moment coefficients. Thus, there are generally uncertainties due to force contributions from the fuselage, nacelles, and leading-edge slats. The results presented in this report attempt to account for these additional factors in the following manner.

Corrections. - The predicted aerodynamic characteristics obtained from the USB method described in this report are for a wing-flap-jet configuration only. No fuselage, nacelle, or leading-edge slat effects are included. Generally, these effects are not negligible and should be be included with the predictions to give meaningful comparisons with the data. Thus, the following corrections are made to the predictions. 
The total lift coefficient is

$$
C_{L}=C_{L_{W F}}+C_{\mu} \sin \alpha+C_{L_{B}}
$$

where the first term is from the wing-flap vortex-lattice method with jet interference, and the second term is the component of total thrust in the lift direction. The last term in equation (11) is the combined lift of the leading-edge slat, the fuselage, and the nacelles.

The total pitching-moment coefficient is

$$
c_{m}=c_{m_{W F}}+c_{m_{B}}+c_{\mu} \frac{\left(z_{Q}-z_{m}\right)}{l_{\text {ref }}}-c_{D_{\text {ram }}} \frac{\left(z_{Q}-z_{m}\right)}{l_{r e f}}
$$

where $C_{m_{W F}}$ is the result from the vortex-lattice prediction method. $C_{m_{B}}$ is the pitching-moment due to the fuselage, nacelles, and leading-edge slat. The third term is the engine thrust contribution to the total pitching-moment, and the last term is the engine ram drag contribution. Although some of these components to the total pitching moment are small in magnitude compared to the wing-flap contribution, they can have an appreciable effect on the moment curve slope.

The total (nonviscous) drag coefficient produced by the prediction method is

$$
C_{D}=C_{D_{W F}}+C_{D_{r a m}}-C_{\mu} \cos \alpha
$$

where $C_{D_{W F}}$ is the induced drag coefficient on the wing and flaps calculated by the vortex-lattice method. The value $\mathrm{C}_{\mathrm{D}_{\text {ram }}}$ is the ram drag coefficient for the engines. The last term is the component of total thrust opposing the drag of the model and is included in the predictions to put them in the same form as the data. No estimate of viscous drag is included in the prediction method.

The authors have investigated several different methods for predicting the unknown correction quantities in equations (11), (12), and (13). The leading-edge slat contribution to the forces and moments is generally very small and difficult to predict accurately. The effect of the leading-edge slat is neglected in the corrections used in this report, but if the user has information on slat loads from predictions or experiment, these loads should be included. The fuselage and nacelles contributions to the forces and moments have been predicted using three different methods. The simplest procedure assumes these body components 
to be axisymmetric and applies slender-body theory (ref. 18) to predict the lift force and center of pressure. A second method assumes the fuselage and nacelles to be extensions of the wing and models them with a vortex lattice lifting surface with the same planform shape as the fuselage and nacelles. A third approach requires the use of experimental data. The fuselage and nacelles contribution to the forces and moments was taken as the difference between wing-body-nacelles experimental results and wing alone predicted results for zero flap deflection angle and zero thrust. All three of these approaches produced similar fuselage and nacelle forces. The first approach, being the easiest and most general to use, was selected to predict the fuselage and nacelles corrections used in the predicted results to follow.

The ram drag factor in equation (13) is often specified with engine characteristics as a function of thrust coefficient. When it isn't specified directly, it can be calculated assuming the ram drag to be the free stream momentum of the mass of air entering the engine inlet per second. For most cases it is acceptable to assume the mass flow at the exit to equal the mass flow at the inlet.

Data available. - A large quantity of USB model experimental results are available for comparison purposes. Most of these data consist of gross force and moment coefficients. For purposes of evaluating the USB prediction method, more detailed information on the distribution of loading is desirable. The data of reference 13, consisting of pressure data, span load distributions, and gross forces and moments on a reasonably clean configuration, is the best data available for initial comparisons. Because these data are unique, they will be used almost exclusively to evaluate the prediction method and the associated computer program. After the initial evaluation and discussion of the results on the model from reference 13, the method will be applied to two other configurations (references 14 and 19) to demonstrate the general use of the method.

The USB model of reference 13 is a large-scale, two-engine configuration with an aspect ratio 5.76 wing having an unswept leading edge and a 0.47 taper ratio. A full-span Krueger flap with a chord equal to $.20 \mathrm{c}_{\mathrm{w}}$ is attached to the wing leading edge at a constant $76^{\circ}$ deflection angle. The trailing edge flap system consists of three spanwise regions: an inboard Coanda flap located behind the engine, a mid-span double slotted 
flap, and outboard ailerons. The Coanda flap has a smooth, large-radius, continuously curved upper surface which is a continuation of the wing upper surface. The JT15D-1 turbofan engine on each semispan is housed in a nacelle mounted above the wing at the $\eta=0.21$ semispan station. The exhaust nozzle is rectangular with an aspect ratio of 6 . Tests were run at gross thrust coefficients between 0 and 4 , and two coanda flap deflection angles, $32^{\circ}$ and $72^{\circ}$, are considered. More detailed configuration data are available in reference 13 .

A second set of data used for comparison purposes is that for the four-engine USB model of reference 19. This model has an aspect ratio 7.48 wing with a taper ratio of 0.247 and a quarter chord sweep angle of $30^{\circ}$. The 15-percent chord leading-edge slat has a constant deflection angle of $40^{\circ}$, and the trailing edge flaps system consists of two regions. The inboard region is a simple radius flap (Coanda flap) with deflection angles of $0^{\circ}, 45^{\circ}, 60^{\circ}, 75^{\circ}$, and $90^{\circ}$. The outboard region contains a single, cambered, undeflected flap. Two engine nacelles are mounted on the wing upper surface at semispan stations $n=0.254$ and 0.417 . Air ejectors provide engine simulation with a high-pressure air supply, and the exhaust nozzles are aspect ratio 6 rectangles. Measurements in the jet wake of the inboard engine are provided in reference 15. Additional configuration information is available in reference 19.

A third set of data used for comparison purposes is that for the two-engine USB model of reference 14. This model has an aspect ratio 7.28 wing with a quarter chord sweep of $25^{\circ}$. A full span leading-edge slat was deflected $50^{\circ}$ for all tests. The trailing-edge flap system is made up of three regions: the inboard region is a Coanda surface, the center region is a double slotted flap, and the outboard region contains a single aileron deflected $20^{\circ}$. Only the $30^{\circ}$ Coanda flap deflection data are considered in this report. The JT15D-l engine is housed in a nacelle mounted on the upper wing surface at the $n=.256$ semispan station. A rectangular exhaust nozzle having an aspect ratio of 3.7 was used for all comparisons with data. Additional configuration information is available in reference 14 .

\section{Data Comparisons}

The first USB configuration on which comparisons with data are made is the two-engine model described in reference 13. The lowest flap deflection angle, $32^{\circ}$, is chosen for the initial predictions; and as 
dictated by figure $10(b)$ of part I of reference 13, the jet turning efficiency is assumed to be 1.0 for all calculations. The jet wake model has a rectangular cross section over its entire length, and the expansion schedule for the boundary is obtained using figures 5 and 8 coupled with the requirement that the cross section aspect ratio be constant over the jet length. The vortex lattice arrangement for this model is shown in figure 11 .

In figure $12(a)$ the predicted section normal-force coefficients on the lifting surface are compared with experimental results at $\alpha \approx 10^{\circ}$ and $C_{\mu}=0$ and 2. The power-off results are in good agreement even though the predicted span loads tend to be slightly larger than those measured. The dip in the measured curve at $n<.3$ occurs because no pressure measurements were made in the nacelle region of the wing. The predicted loads on the same portion of wing were omitted from the span load calculations so that comparable surfaces are considered for both experiment and theory. The power-on results in this figure show the predicted loads on the surfaces aft of the nozzle to be very good agreement except near the inboard half of the nozzle. It appears that the jet may have separated from the upper surface in this region. On the portion of the Coanda flap outboard of the nozzle, the measured and predicted loadings are in good agreement indicating that the jet model has the correct spreading characteristics. The predicted loading on the outboard portion of the wing is larger than that measured. In figure 12(b), measured and predicted section normal force distributions are compared at $C_{\mu}=4$ and $\alpha=26.5^{\circ}$. These results have the same character as those described in the previous figure.

The predicted distribution of section normal force coefficient in figure 12 indicate a discontinuity in loading at the spanwise stations where there is a change in flap geometry, $n \approx 0.4$ and 0.7 . The predicted results presented in this report will show a smooth curve through these discontinuities. These characteristics are not seen in the experimental loadings because of the limited number of semispan stations at which pressures were measured. Pressure data are presented in reference 13 at wing semispan stations $n=.147, .188, .214, .357, .540$, and .835. The section normal force results presented in the same reference and used herein were obtained from integration of these pressure data. 
In addition to span load distributions, the pressure difference across each lifting surface panel is also obtained from the calculation. Since some pressure data are available in reference 13, comparisons between measured and calculated pressure distributions are shown in figure 13. Comparisons of this type are useful in evaluating the ability of the method to predict the chordwise loading on the wing and flaps. The chordwise pressure distributions at four semispan stations are presented. The inboard station is at the engine centerline, and each of the other three stations corresponds to one of the flap regions on the wing. The knee of the flap occurs at $x / c=.75$ in these figures. The power-off results are shown in figure 13(a), where it can be seen that the predicted pressure loading is higher on the flaps than that measured. The measured and predicted loadings on the wing are in good agreement at all semispan stations except that portion of the wing aft of the nacelles. In this region the presence of the nacelle has an effect on the wing loading that is not included in the prediction method. Power-on results are shown in figure $13(\mathrm{~b})$ for approximately the same angle of attack as the previous figure and at $\mathrm{C}_{\mu} \approx 2$. As before, the wing loading is in good agreement, but the predicted flap loading is lower than that measured at the two stations nearest the jet. At the station near the jet centerline, $n=.214$, data are not available near the knee of the flap, but the predicted flap loading is concentrated toward the flap leading edge, contrary to the appearance of the data. At $n=.357$, the predicted flap loading shows very little influence of the jet because the jet model did not expand laterally far enough for this station to be covered by the jet.

The measured and predicted longitudinal aerodynamic characteristics of this USB model with $32^{\circ}$ Coanda flap are compared in figure 14. The experimental points shown in this figure are taken from the faired data curves in figure $15(\mathrm{e})$ of reference 13. The predicted lift, pitching moment, and drag results are obtained from equations (11), (12), and (13), respectively. The predicted power-off lift curve has less slope than the experimental curve, but the lift at $\alpha=0^{\circ}$ is overpredicted. The corresponding pitching-moment curve is in good agreement with experiment; however, the tendency is for the predicted results to have a larger nosedown moment than the data. The power-off drag curve is lower than the data as would be expected since viscous drag is not considered in the prediction method. 
The predicted lift coefficient curve for power-on conditions generally exhibits less slope than the data, but the zero angle lift coefficient is always larger than that measured. This condition gets slightly worse at the higher thrust levels. The predicted pitching moment results are in good agreement at low angles of attack, but they are too large, in the nose-down direction, at higher angles of attack. The drag coefficient is consistently underpredicted.

The quality of the comparisons shown in figure 14 are strongly dependent on the quality of the estimated fuselage and nacelle forces and moments. These estimated quantities can be removed in the following manner. If we assume the fuselage lift force to be independent of engine thrust coefficient, the jet induced effects on the wing-flap can be isolated using a $\Delta C_{L}$ defined as follows.

$$
\Delta C_{L}=\left.C_{L}\right|_{C_{\mu}}>0-\left.C_{L}\right|_{C_{\mu}}=0
$$

The $\Delta C_{L}$ curves developed using only predicted results and only experimental results are shown in figure 15. These results should contain the total jet induced lift effects, including the supercirculation on the wing and flaps. It is interesting to note that the experimental and theoretical results agree within 10-percent over the entire range of thrust coefficient and angle of attack. Some divergence of the curves is starting at angles of attack above $20^{\circ}$, but this is caused by the dropoff of the experimental lift curves due to separation.

Experimental data on this same two-engine USB model with Coanda flap deflected $72^{\circ}$ are also available in reference 13 . In addition to overall force and moment coefficients, span load and chordwise pressure distributions are presented. A vortex lattice arrangement similar to that shown in figure 11 is used for the $\delta_{f}=72^{\circ}$ calculations. The three flap segments making up the coanda flap are extended in chord and the deflection angles increased to suitably model the new configuration. As shown in figure $10(a)$ of Part $I$ of reference 13, the jet turning efficiency is approximately 0.9 for all thrust levels. The jet wake expansion model is unchanged from the previous calculations.

Measured and predicted section normal-force coefficients are compared for a wide range of flow conditions in figure 16. In figure 16(a), the effect of thrust coefficient at a nearly constant angle of attack $\left(\alpha \approx 10^{\circ}\right)$ is investigated. The power-off results are in good agreement 
over the total span of the wing. The power-on results for $c_{\mu} \approx 2$ are in agreement near the outboard half of the nozzle, but the predicted loading is generally too high outboard of the nozzle. At the highest thrust level, $\mathrm{C}_{\mu} \approx 4$, the predicted section normal force coefficients are too large over the entire span of the wing. As noted on the previous configuration, the erratic loading near the inboard half of the jet may be caused by separation of the jet from the wing upper surface.

The effect of angle of attack, at constant thrust coefficient $\left(C_{\mu}=2.5\right)$, on the spanwise distribution of section normal-force coefficient is shown in figure 16(b). The comparisons between measured and predicted loadings have the same appearance at all angles of attack in that the peak loadings in the vicinity of the jet wake are in good agreement, but the loading on the outboard portion of the wing is generally overpredicted. The prediction method is successful in predicting the general effect of angle of attack on the spanwise load distributions as it correctly predicts the trend of the results. There is only a small effect of angle of attack on the level of the loading, but as was seen in the previous figure, there is a large effect of thrust coefficient on the level of the loading.

In figure 16(c), comparison of the measured and predicted span load distribution at $C_{\mu} \approx 4$ and $\alpha \approx 28^{\circ}$ indicate the predicted loading levels to be much higher than those measured. This result is similar to that observed at a lower angle of attack in figure 16 (a).

Measured and predicted pressure distributions are shown in figure 17 for three thrust coefficients; $C_{\mu}=0,2$, and 4 . In figure $17(a)$, the power-off results show that the predicted pressure difference on the wing is in good agreement with experiment at all semispan stations except $\eta=0.214$, which is downstream of the exhaust nozzle. As mentioned before, the presence of the nacelle may be causing this lack of agreement as the prediction method does not include the effect of the nacelle. On the flaps, the predicted pressure difference is typically less than that measured. The power-on results shown in figures $17(b)$ and (c) are similar to the low flap angle results presented in figure 13(b). At the station aligned with the engine centerline, $n=.214$, the predicted loading peak is concentrated toward the leading edge of the Coanda flap, but the data indicate the loading peak to be aft on the flap and spread out over the entire flap. At the second semispan station, $n=.357$, the effect of not 
allowing the jet model to spread laterally enough to cover the entire Coanda flap is seen in the comparison with data. The predicted loading on the flap is much less than that measured, and the character of the loading is that of a wing-flap section with no direct jet interference. The comparisons on the outboard semispan stations show the same type of agreement as seen on the lower flap angle configuration in figure 13.

The measured and predicted longitudinal aerodynamic characteristics of the same USB model with 72-degree Coanda flap are compared in figure 18. The experimental points shown in this figure are taken from the faired data curve in figure 12 (c) of reference 13. The predicted results presented on this figure are similar to those shown for the lower flap deflection angles in figure 14. The power-off lift curve is above the measured lift at $\alpha=0^{\circ}$, but agreement is very good at $\alpha \geq 10^{\circ}$. The predicted pitching moment curve has the correct slope, but the predicted pitching moments are more nose-down than those measured. The predicted induced drag of the power-off configuration is much lower than the total measured drag.

The predicted lift results with power-on are in good agreement at angles of attack less than $20^{\circ}$ and $c_{\mu} \leq 2$. At higher angles of attack, the measured lift curves exhibit a large amount of nonlinearity, probably due to flow separation. The predicted lift results for $c_{\mu}=3$ are about 8-percent higher than the measured lift results.

The pitching moment results shown in figure 18 have about the correct magnitude at the lower lift levels, but the moment curve slope is not predicted well. As before, the predicted pitching moments at angles of attack greater than ten degrees are more nose-down than those measured.

The drag results in this figure show poor agreement under all thrust conditions. For this reason, only results for $c_{\mu}=0$ and 1 are presented.

Analogous to figure 15 for the low flap deflection angle, the jet induced lift for the high flap deflection angle is presented in figure 19. It can be seen that the jet induced effects are predicted within tenpercent at angles of attack less than $15^{\circ}$ and thrust coefficients of two or less. Figures 15 and 19 show that the theory predicts for both moderate and high flap angles a significant increase in lift coefficient with angle of attack which is verified by the measurements only at the 
moderate flap angle. At the high flap angle, there is only a small measured effect of angle of attack on lift.

For the second set of comparisons, the USB prediction method was applied to the four-engine USB configurations of reference 19. The flap angles chosen for comparison with data are $0^{\circ}, 45^{\circ}$, and $60^{\circ}$, and these comparisons are presented in figure 20. The configuration denoted as $\delta_{f}=0^{\circ}$ in figure $20(a)$ corresponds to a USB model in a cruise configuration. This configuration is modeled as a wing with a flap along the 0.75 percent chord line. The deflection of this flap was specified to be approximately equal to the slope of the upper wing surface at the trailing edge. The predicted power-off lift and pitching moment results are in good agreement with data until the lift curve begins to drop off at about $12^{\circ}$ angle of attack. The predicted power-on results are also in good agreement with the experimental results. The lift at zero angle of attack is too high at higher thrust levels, and the lift curve slope is too low for all power-on conditions. The pitching moment curves are in good agreement with the experimental results for all conditions.

Measured and predicted force and moment coefficients are compared in figures 20 (b) and (c) for the configuration with coanda flaps deflected $45^{\circ}$ and $60^{\circ}$, respectively. The agreement between the measured and predicted lift and pitching moment curves is good at thrust coefficients of one or less, but at higher thrust levels the lift is overpredicted by a large amount. There is an associated over prediction of nose-down pitching moment coefficient. In each of these cases, the turning efficiency at all thrust levels was taken to be approximately 0.65 as indicated by the data in reference 19 .

The jet-induced lift coefficients on this four-engine USB configuration at various thrust levels are shown in figure 21 for three flap deflection angles. These results are similar to those of the two-engine model (figures 15 and 19) with several exceptions. In the cruise condition $\left(\delta_{f}=0^{\circ}\right)$ there is no systematic variation with thrust level. For the two high flap deflection configurations, the experimental jet-induced lift is a strong function of angle of attack. The difference between the measured and predicted jet-induced lift is nearly constant with angle of attack and appears to vary only with thrust coefficient. This is in direct contrast with figures 15 and 19 for the two-engine configuration where the measured and predicted jet-induced lift coefficients are in better agreement at low angles of attack. These facts might indicate 
that the four-engine model turning efficiency was constant with angle of attack, and the turning efficiency of the two-engine model decreased at higher angles of attack.

It appears from the high thrust level results in figure 21 that the chosen turning efficiency used in the prediction was too high. A sample calculation on the $\delta_{f}=45^{\circ}$ model at $C_{\mu}=4$ was made using an assumed turning efficiency of 0.50. The predicted lift and pitching moment results were in much better agreement with experiment. The difference between the measured and predicted jet-induced lift coefficients in figure 21 was half that shown in the present figure. Even though these results are indicative that a lower turning efficiency should be used in the predictions to improve agreement between experiment and theory, there is no rational basis for choosing a lower turning efficiency.

In the third set of comparisons, the USB prediction method was applied to the two-engine configuration of reference 14 with Coanda flap deflected $30^{\circ}$. Comparison of measured and predicted longitudinal aerodynamic characteristics is made in figure 22 for a range of thrust conditions. As has been typical of the results presented in this section, the lift curves are in good agreement at low thrust levels. As thrust increases, the predicted lift is greater than that measured. Generally, the pitching moment results are in good agreement when the lift is in good agreement. The drag results are also shown in this figure, but as would be expected, the predicted drag results are always lower than those measured.

The incremental lift due to jet-induced effects is shown in figure 23. Here it can be seen that the predicted lift increment due to the presence of the jet is predicted within 10-percent at $c_{\mu} \leq 1$. As discussed with respect to figure 21, the lift increment is overpredicted by a constant amount at the two highest thrust coefficients. This may indicate an error in the assumed jet turning efficiency. A static turning efficiency of 0.9 was presented in reference 14 for a single thrust level. This value was used for all calculations described above.

\section{CONCLUDING REMARKS}

An engineering prediction method has been developed to predict the loading distributions and longitudinal aerodynamic characteristics of upper surface blown flap configurations. Two potential flow models are 
combined for the method. The first is a vortex-lattice lifting-surface method capable of representing a wing of arbitrary sweep, taper, dihedral, camber, and twist with large, partial or full-span flaps which can have large deflection angles. The second is a vortex ring model of a jet wake from an upper surface mounted turbofan engine which represents the mass, momentum, and spreading of a turbulent jet with rectangular cross section. The jet is positioned a priori tangent to the upper wing and flap surfaces. These models are combined to predict the loading on the wing and flaps in the presence of the jet wakes. The method yields chordwise pressure distributions and span load distributions on each lifting surface and the total forces and moments on each surface and the configuration as a whole. Use of the method is restricted to the case of zero sideslip and symmetry about the vertical plane through the wing root chord.

The complete prediction method was applied to USB configurations on which detailed aerodynamic data are available. The comparisons of measured and predicted gross lift and pitching-moment coefficients on USB configurations with a wide range of flap angles $\left(0^{\circ} \leq \delta_{f} \leq 72^{\circ}\right)$ indicate generally good agreement for low thrust levels. This is felt to be due principally to the correct modeling of the general characteristics of the engine wakes and to the proper treatment of the mutual interference between the jet wake and wing-flap. The interference model creates, on the wing-flap, both the momentum reaction due to jet deflection and the additional induced circulation characteristic of upper surface blown flap systems. At low thrust levels, the jet wake may remain more fully attached to the upper wing and flap surfaces and thus, the potential models of the wake and lifting surfaces are good representations of the actual flow conditions. At higher thrust levels, there is a possibility that the jet wake does not remain attached to the flap surface as long, thus, the turning efficiency is decreased and the jet induced effects are reduced. The fact that the predicted pitching moments are too negative tends to support this possibility, since unloading the flap somewhat would produce less nose-down moments. There is also a possibility that the turning efficiency of a particular wing-flap combination is a function of the angle of attack of the model; however, data are not available to verify this possibility.

Comparisons of measured and predicted spanwise distributions of section normal force on two USB configurations indicate good quantitative agreement in most cases. The magnitude and position of the large peak 
loadings induced by the jet wake on the wing and flap surfaces are generally in good agreement with data. The differences in many cases are due to the separation of the jet from the upper wing and flap surfaces which can cause localized losses of lift. Outside the direct influence of the jet wake, the comparisons of measured and predicted span loading distributions generally indicate the predicted loading to be greater than that measured.

The loadings predicted by the USB method described in this report are not sensitive to the lattice layout on the wing and flaps. It is necessary that some care be used in setting up a lattice arrangement but an extremely fine lattice is not required in the vicinity of the jet boundary .

The rectangular vortex ring jet wake model can be set up to match the mass and momentum of an actual USB jet. Comparisons with data show good agreement near the exhaust nozzle where the initial uniform velocity profile of the actual jet has not decayed. Comparisons with data at a station on the flap indicate some differences between the measured and predicted jet characteristics. If an adequate quantity of experimental information on the USB jet wake is available, it is possible to tailor the vortex ring jet model to produce a good analytical representation of the actual jet over its entire length.

The predicted pressure distributions on the lifting surfaces in the region of maximum jet interference show the correct general trends, but the use of only a small number of discrete constant pressure panels prevents the prediction of the details of the pressures. Power-off pressure distributions are generally predicted quite well. Power-on pressure distributions generally do not agree well on the flap. The pressure peaks have the correct magnitude, but their location is generally more forward on the surface than is indicated by the measured results.

The preceding comments generally indicate that the overall momentum reaction and jet induced circulation effects are properly modeled by the USB prediction method. The results are best at moderate flap angles and low thrust coefficients. The availability of more detailed experimental information on the flow on the wing and flap upper surfaces in the vicinity of the jet wake would allow more accurate modeling of the jet which would improve the predicted results at high thrust conditions. 
Nielsen Engineering \& Research, Inc.

Mountain View, California 94043

March 1978 


\section{APPENDIX A \\ INDUCED VELOCITY FIELD DUE TO A QUADRILATERAL VORTEX RING}

The following analysis for the velocity field induced by a single quadrilateral vortex $r i n g$ is reproduced from reference 11 . Consider a single quadrilateral vortex ring as shown in sketch $A l$.
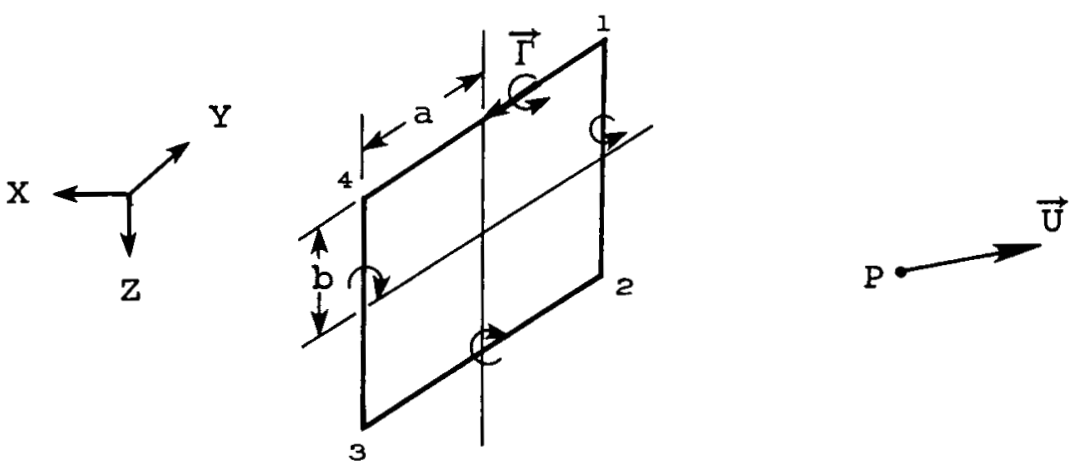

\section{SKETCH Al.- QUADRILATERAL VORTEX RING}

According to the sign convention chosen for the jet model, a positive $\Gamma$ is indicated in the above sketch. Consider now the contribution of side 1-2 to the velocity at point P. Sketch A2, also from reference 11, illustrates a single side of the vortex ring and identifies the parameters required for the derivation of the velocity equations.

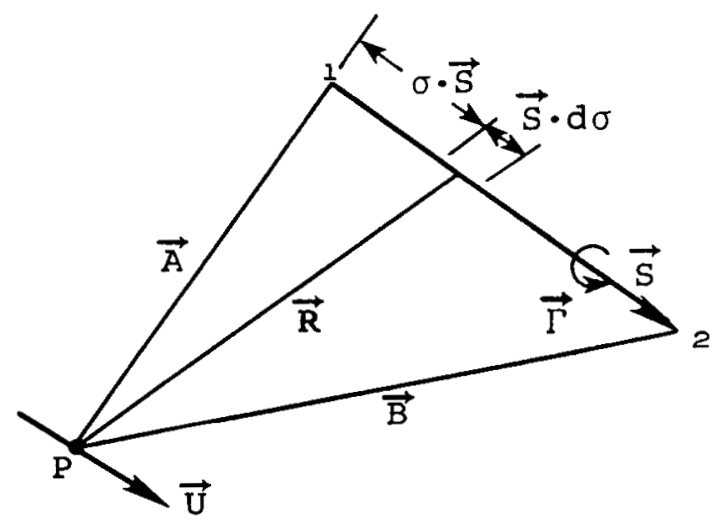




$$
\vec{U}=\frac{1}{4 \pi} \int_{\vec{A}}^{\vec{B}} \frac{\vec{R} \times \Gamma \overrightarrow{\mathrm{dS}}}{|\vec{R}|^{3}}
$$

where $\Gamma$ is the density of vorticity per unit length.

$$
\begin{aligned}
& \vec{R}=\vec{A}+\sigma \vec{S} \\
& \overrightarrow{d S}=\vec{S} d \sigma \\
& \vec{U}=u \vec{I}+v \vec{j}+w \vec{k}
\end{aligned}
$$

The resultant velocity at point $P$, from equation (AI) is

$$
\vec{U}=\frac{\Gamma}{4 \pi} \frac{\vec{A} \times \vec{B}}{(\vec{A} \times \vec{B}) \cdot(\vec{A} \times \vec{B})}[|\vec{A}|+|\vec{B}|]\left[1-\frac{\vec{A} \cdot \vec{B}}{|\vec{A}||\vec{B}|}\right]
$$

Let

$$
\begin{aligned}
& \vec{A}=\left(X_{1}-X_{P}\right) \vec{i}+\left(y_{1}-y_{P}\right) \vec{j}+\left(z_{1}-z_{P}\right) \vec{k} \\
& \vec{B}=\left(X_{2}-X_{P}\right) \vec{i}+\left(y_{2}-y_{P}\right) \vec{j}+\left(z_{2}-z_{P}\right) \vec{k}
\end{aligned}
$$

It follows that

$$
\begin{aligned}
& \vec{A} \times \vec{B}=\left[\left(Y_{1}-Y_{P}\right)\left(Z_{2}-Z_{P}\right)-\left(Y_{2}-Y_{P}\right)\left(Z_{1}-Z_{P}\right)\right] \vec{i} \\
& -\left[\left(x_{1}-x_{P}\right)\left(z_{2}-z_{P}\right)-\left(x_{2}-x_{P}\right)\left(z_{1}-z_{P}\right)\right] \vec{j} \\
& +\left[\left(X_{1}-X_{P}\right)\left(Y_{2}-Y_{P}\right)-\left(X_{2}-X_{P}\right)\left(Y_{1}-Y_{P}\right)\right] \vec{k} \\
& =A_{Y Z} \vec{i}+A_{X Z} \vec{j}+A_{X Y} \vec{k} \\
& (\vec{A} \times \vec{B}) \cdot(\vec{A} \times \vec{B})=A_{Y Z}^{2}+A_{X Z}^{2}+A_{X Y}^{2} \\
& \vec{A} \cdot \vec{B}=\left(X_{1}-X_{P}\right)\left(X_{2}-X_{P}\right)+\left(Y_{1}-Y_{P}\right)\left(Y_{2}-Y_{P}\right) \\
& +\left(z_{1}-z_{P}\right)\left(z_{2}-z_{P}\right) \\
& |\vec{A}|=\left[\left(x_{1}-x_{P}\right)^{2}+\left(y_{1}-y_{P}\right)^{2}+\left(z_{I}-z_{P}\right)^{2}\right]^{1 / 2} \\
& |\vec{B}|=\left[\left(x_{2}-x_{P}\right)^{2}+\left(y_{2}-y_{P}\right)^{2}+\left(z_{2}-z_{P}\right)^{2}\right]^{1 / 2}
\end{aligned}
$$


From (A5),

$$
\vec{U}=K\left(A_{Y Z} \vec{i}+A_{X Z} \vec{j}+A_{X Y} \vec{k}\right)
$$

where

$$
K=\frac{-\Gamma}{4 \pi} \frac{\{|\vec{A}|+|\vec{B}|\}\left[1-\frac{\vec{A} \cdot \vec{B}}{|\vec{A}||\vec{B}|}\right]}{(\vec{A} \times \vec{B}) \cdot(\vec{A} \times \vec{B})}
$$

Note that the sign of $K$ depends on the direction of integration around the ring. The sign of equation (Al4) is appropriate to the sign convention of the single ring shown in sketch Al. It follows that the components of velocity induced by the single leg of the vortex ring are

$$
\begin{aligned}
& \overrightarrow{\mathrm{u}}=K \mathrm{KA}_{\mathrm{YZ}} \overrightarrow{\mathrm{i}} \\
& \overrightarrow{\mathrm{v}}=\mathrm{KA} \mathrm{XZ}_{\mathrm{X}} \overrightarrow{\mathrm{j}} \\
& \overrightarrow{\mathrm{w}}=\mathrm{KA} \mathrm{XY}_{\mathrm{k}} \overrightarrow{\mathrm{k}}
\end{aligned}
$$

The total velocity at point $P$ induced by the complete ring is obtained by summing the contributions of the four sides. The four sides are taken in the order shown in sketch $A l$, starting at corner 1 and proceeding around the ring such that the last side is that side connecting corner 4 with corner 1 . 


\section{REFERENCES}

1. Lopez, M. L. and Shen, C. C.: Recent Developments in Jet Flap Theory and Its Application to STOL Aerodynamic Analysis. AIAA Paper No. 71-578, June 1971.

2. Lan, C. E. and Campbell, J. F.: Theoretical Aerodynamics of UpperSurface-Blowing Jet-Wing Interaction. NASA TN D-7936, Nov. 1975.

3. Lan, E. C. and Campbell, J. F.: A Wing-Jet Interaction Theory for USB Configurations. J. Aircraft, vol. 13, no. 9, Sept. 1976, pp. 718-726.

4. Mendenhal1, Michael R.: A Computer Program to Calculate the Longitudinal Aerodynamic Characteristics of Upper-Surface-Blown WingFlap Configurations. NASA CR-3005, 1978.

5. Mendenhal1, M. R., Perkins. S. C., Jr., Goodwin, F. K., and Spangler, S. B.: Calculation of Static Longitudinal Aerodynamic Characteristics of STOL Aircraft with Upper-Surface-Blown Flaps. NASA CR137646, Apr. 1975.

6. Mendenhall, M. R., Goodwin, F. K., and Spangler, S. B.: A Computer Program to Calculate the Longitudinal Aerodynamic Characteristics of Wing-Flap Configurations with Externally Blown Flaps. NASA CR2706, Sept. 1976.

7. Rubbert, Paul E.: Theoretical Characteristics of Arbitrary wings by a Non-Planar Vortex Lattice Method. Boeing Report D6-9244, Feb. 1964.

8. Maskew, Brian: A Quadrilateral Vortex Method Applied to Configurations with High Circulation. NASA SP-405, Vortex Lattice Utilization Workshop, May 17-18, 1976, pp. 163-186.

9. Mendenhal1, M. R., Spangler, S. B., Nielsen, J. N., and Goodwin, F. K.: Calculation of the Longitudinal Aerodynamic Characteristics of Wing-Flap Configurations with Externally.Blown Flaps. NASA CR-2705, Sept. 1976.

10. Dillenius, M. F. E., Mendenhall, M. R., and Spangler, S. B.: Calculation of the Longitudinal Aerodynamic Characteristics of STOL Aircraft with Externally-Blown Jet-Augmented Flaps. NASA CR-2358, Feb. 1974 . 
11. Maskew, Brian: Calculation of the Three-Dimensional Potential Flow Around Lifting Nonplanar Wings and Wing-Bodies Using a Surface Distribution of Quadrilateral Vortex Rings. Report TT-7009, Loughborough University of Technology, Sept. 1970.

12. Shivers, James P. and Smith, Charles C., Jr.: Static Tests of a Simulated Upper Surface Blown Jet-Flap Configuration Utilizing a Full-Size Turbofan Engine. NASA TN D-7816, Feb. 1975.

13. Staff of the Langley Research Center: Wind-Tunnel Investigation of Aerodynamic Performance, Steady and Vibratory Loads, Surface Temperatures, and Acoustic Characteristics of a Large-Scale Twin-Engine Upper-Surface Blown Jet-Flap Configuration. NASA TN D-8235, Nov. 1976.

14. Aoyagi, K., Falarski, M. D., and Koenig, D. G.: Wind Tunnel Investigation of a Large-Scale Upper Surface Blown-Flap Transport Model Having Two Engines. NASA TM X-62,296, Aug. 1973.

15. Bloom, A. M., Hohlweg, W. C., and Sleeman, W. C., Jr.: Wing Surface - Jet Interaction Characteristics of an Upper-Surface Blown Model with Rectangular Exhaust Nozzles and a Radius Flap. NASA TN D-8187, Dec. 1976.

16. Carter, A. W.: Pressure Distributions on a Wing Having NACA 4415 Airfoil Sections with Trailing-Edge Flaps set at $0^{\circ}$ and $40^{\circ}$. NASA TM X-2225, June 1971.

17. Küchemann, D. and Weber, J.: Aerodynamics of Propulsion. McGrawHill Book Co., Inc., 1953.

18. Pitts, W. C., Nielsen, J. N., and Kaattari, C. E.: Lift and Center of Pressure of Wing-Body-Tail Combinations at Subsonic, Transonic, and Supersonic Speeds. NACA Report 1307, 1957.

19. Sleeman, William C., Jr. and Hohlweg, William C.: Low-Speed Wind-Tunnel Investigation of a Four-Engine Upper Surface Blown Model Having a Swept Wing and Rectangular and D-Shaped Exhaust Nozzles. NASA TN D-8061, Dec. 1975. 


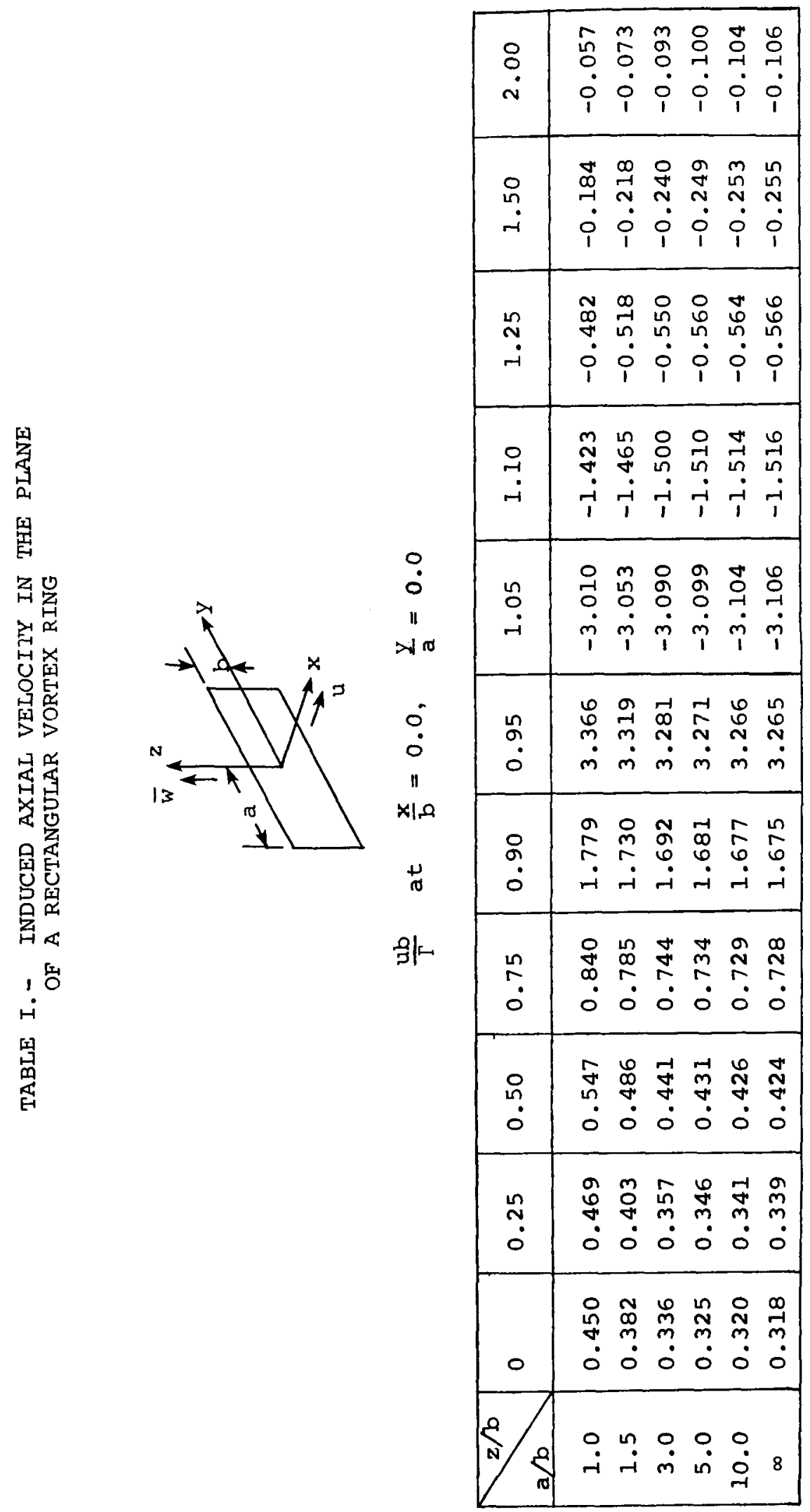




\begin{tabular}{|c|c|c|c|c|c|}
\hline & $\begin{array}{l}8 \\
\dot{i}\end{array}$ & $\begin{array}{llllll}r & m & m & 0 & 0 \\
m & d & 0 & 5 & 0 & 0 \\
0 & 0 & 0 & 0 & 0 \\
\dot{i} & 0 & 0 & 0 & 0 & 0 \\
1 & 1 & 1 & 1 & 1 & i\end{array}$ & & 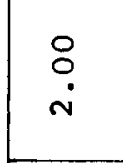 & 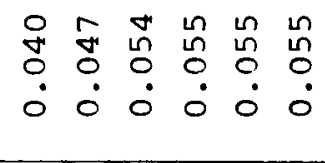 \\
\hline & 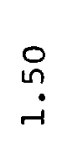 & 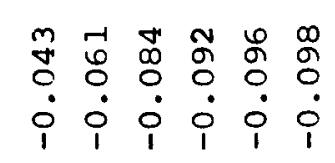 & & $\stackrel{\circ}{\leftrightarrow}$ & 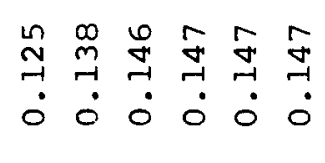 \\
\hline & 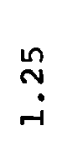 & 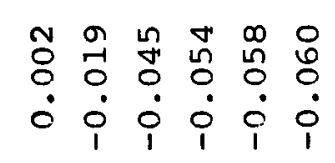 & & $\stackrel{\stackrel{n}{N}}{i}$ & 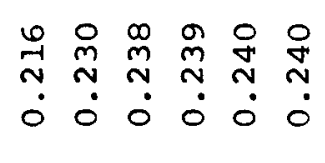 \\
\hline I필 & $\stackrel{\circ}{\stackrel{-}{-}}$ & $\begin{array}{llllll}\hat{n} & m & 0 & 0 & 0 & 0 \\
0 & 0 & 0 & 0 & 0 & 0 \\
0 & 0 & 0 \\
0 & 0 & 0 & 0 & 0 & 0 \\
0\end{array}$ & & $\stackrel{9}{\hookrightarrow}$ & 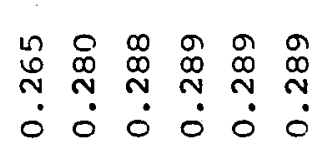 \\
\hline $\begin{array}{l}\dot{0} \\
\| 10\end{array}$ & $\begin{array}{l}\stackrel{n}{\circ} \\
\stackrel{-}{r}\end{array}$ & 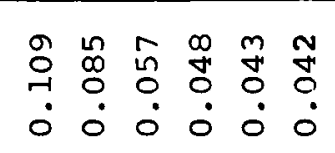 & $\begin{array}{l}: \\
\dot{0} \\
\|>11\end{array}$ & $\begin{array}{l}n \\
\stackrel{n}{0} \\
-i\end{array}$ & 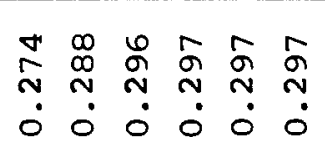 \\
\hline $\begin{array}{l}\dot{n} \\
\dot{0}\end{array}$ & $\begin{array}{l}n \\
\vdots \\
0\end{array}$ & 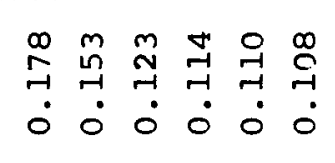 & $\begin{array}{l}\dot{n} \\
\dot{0}\end{array}$ & $\begin{array}{l}n \\
o \\
0\end{array}$ & 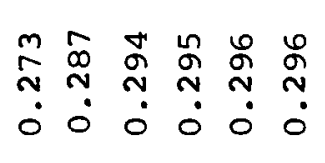 \\
\hline 㟧 & $\begin{array}{l}\stackrel{\circ}{0} \\
0\end{array}$ & 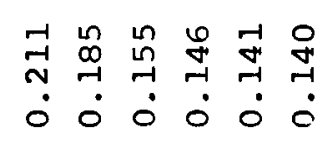 & $\stackrel{+}{\sim}$ & $\begin{array}{l}\delta \\
\dot{\alpha} \\
0\end{array}$ & 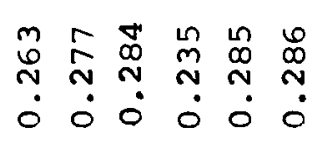 \\
\hline$\exists \mid$ & $\stackrel{n}{\hat{0}}$ & 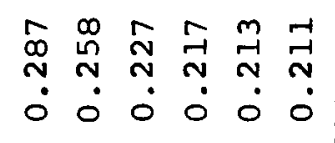 & 影呙 & $\stackrel{n}{\stackrel{n}{0}}$ & 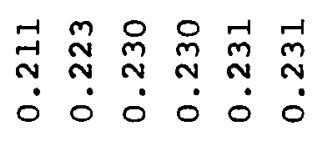 \\
\hline $\bar{n}$ & 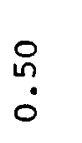 & 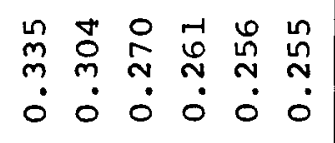 & & $\begin{array}{l}\stackrel{0}{?} \\
\text { on }\end{array}$ & 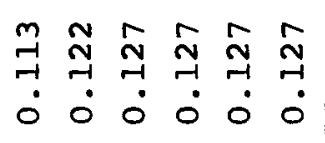 \\
\hline & $\stackrel{\substack{n \\
:}}{\stackrel{0}{0}}$ & 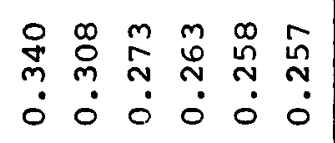 & & 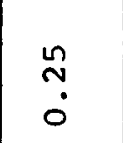 & 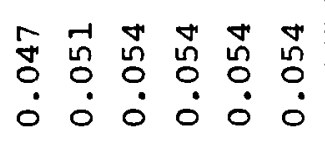 \\
\hline & $\circ$ & 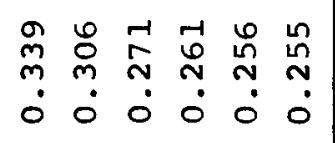 & & $\circ$ & 0000000 \\
\hline & $\int^{\frac{\pi}{2}}$ & 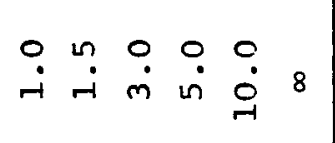 & & $\int^{2}$ & 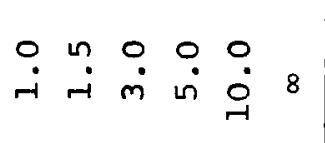 \\
\hline
\end{tabular}




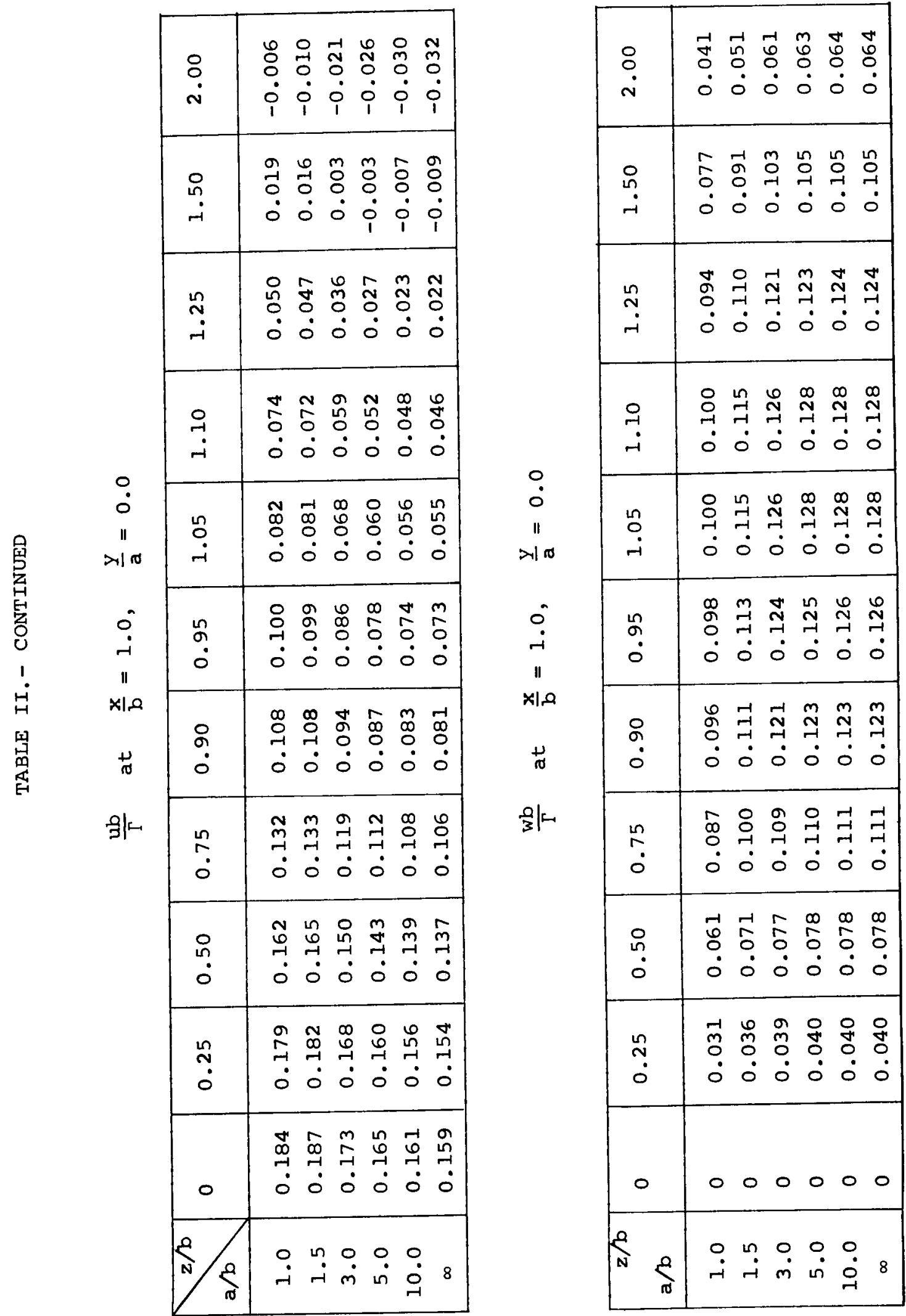




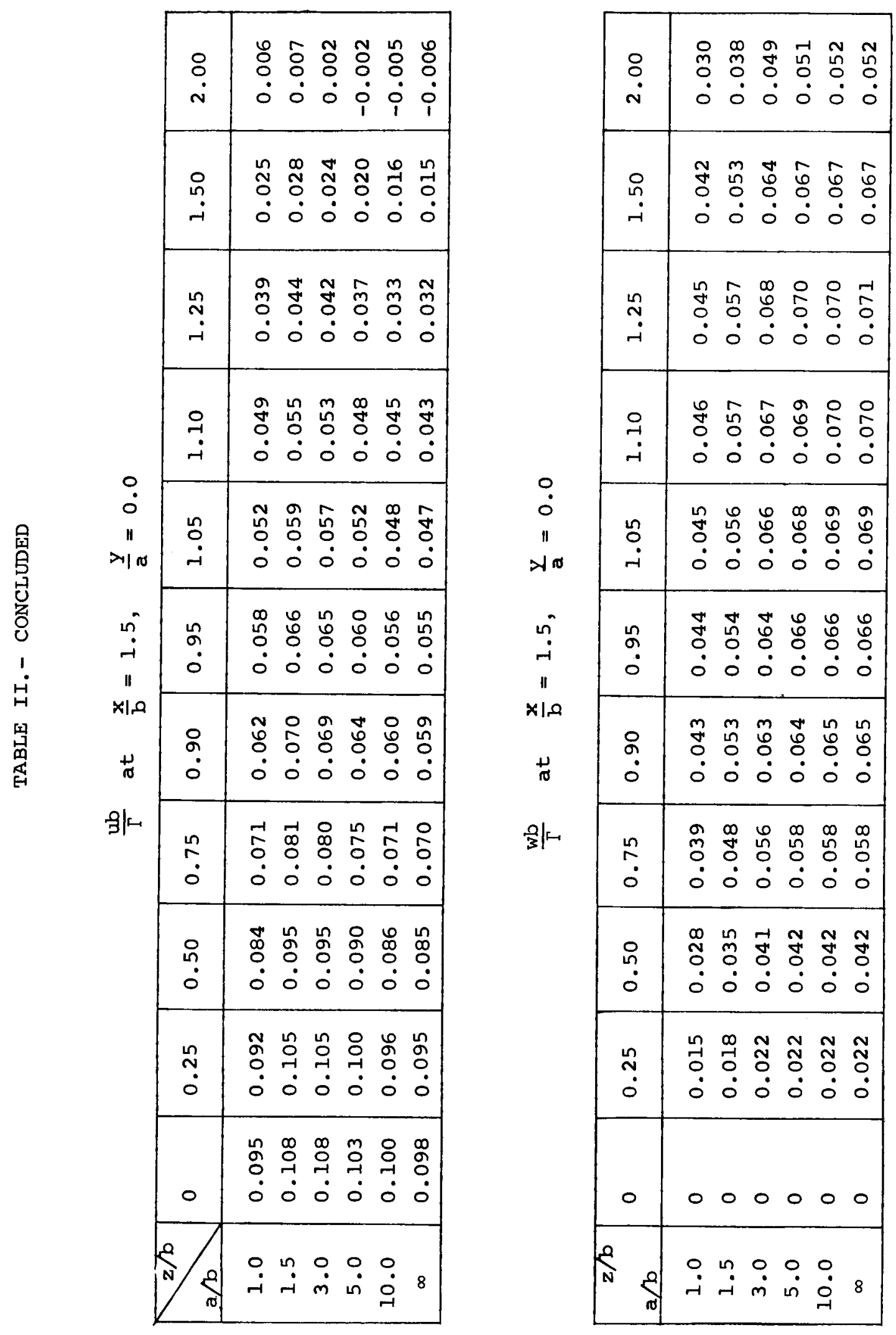




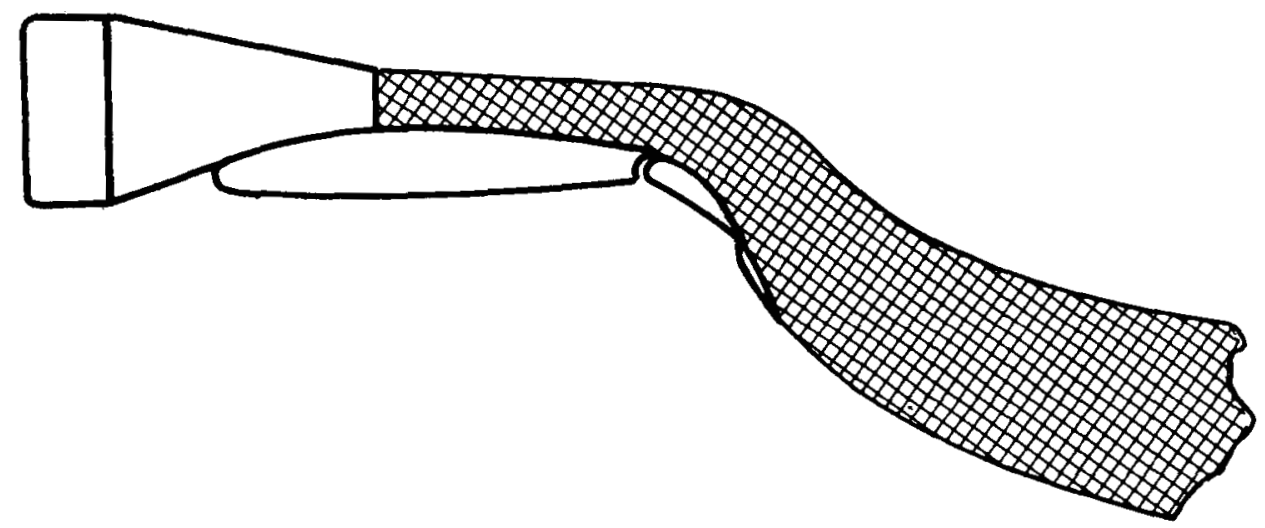

Figure 1.- Upper surface-blown flap configuration. 


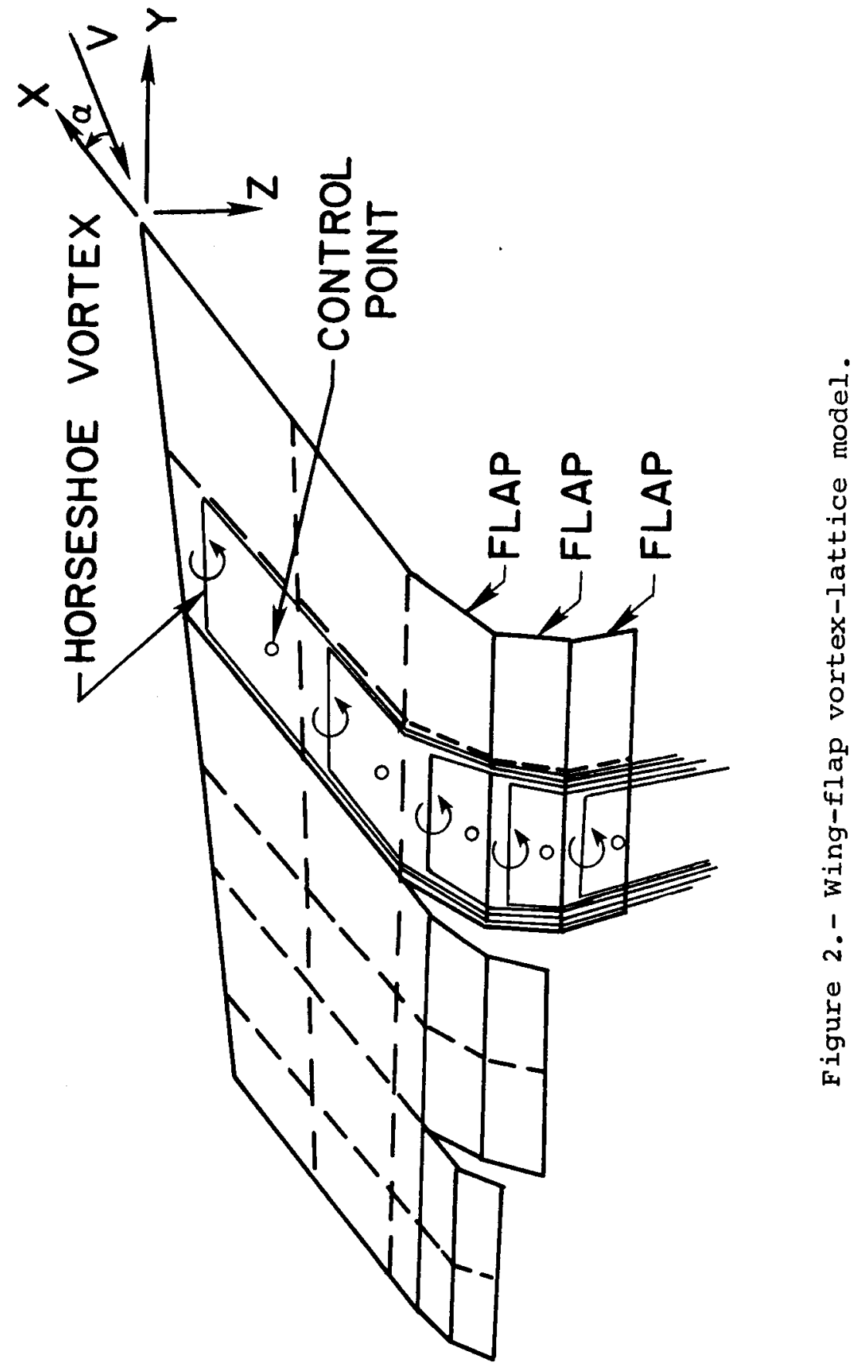




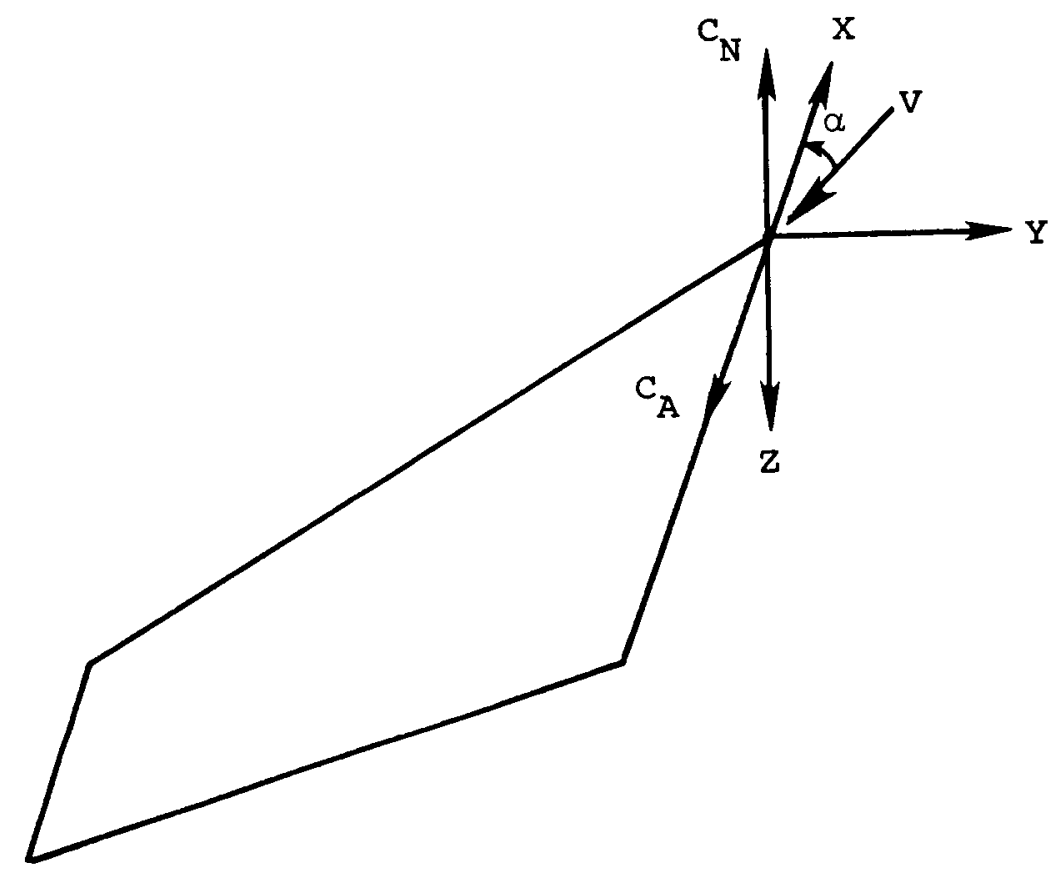

(a) Positive directions of wing forces.

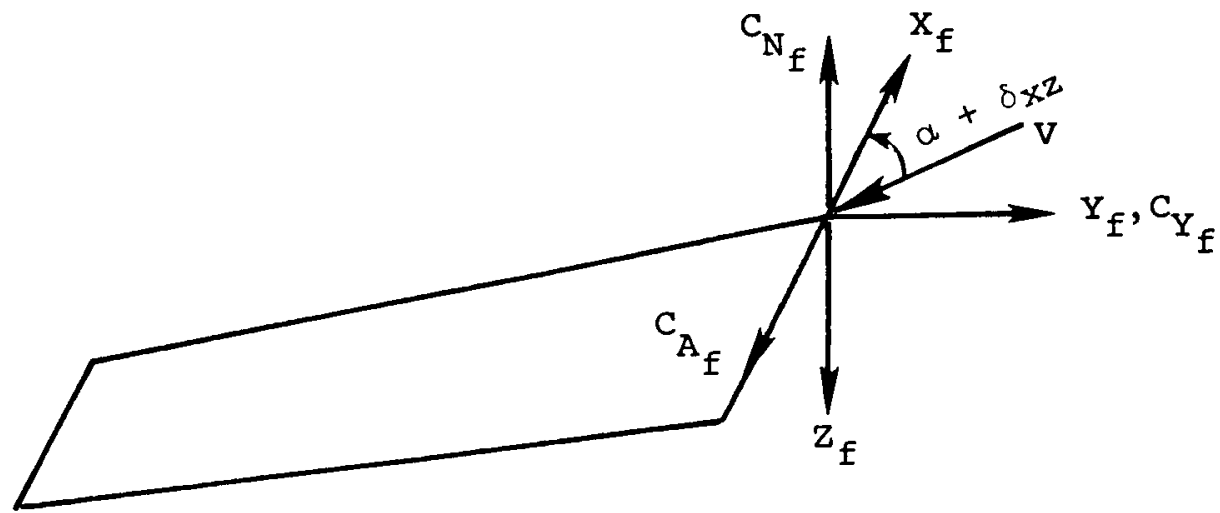

(b) Positive directions of flap forces.

Figure 3.- Force and moment coefficient definitions on individual wing and flap surfaces. 


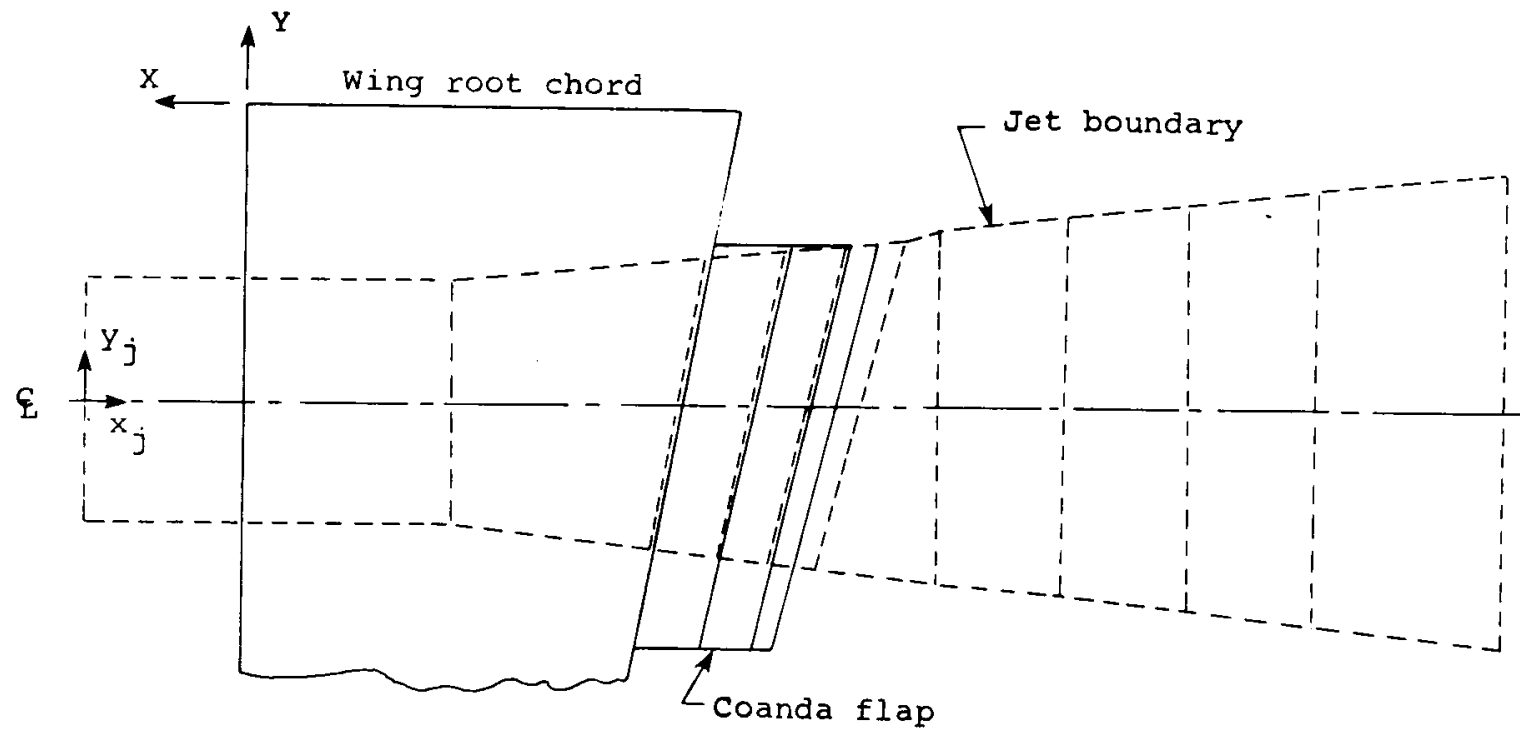

TOP VIEW

$\rightarrow-1 \leftarrow \begin{gathered}\text { Vortex ring } \\ \text { spacing (typ.) }\end{gathered}$

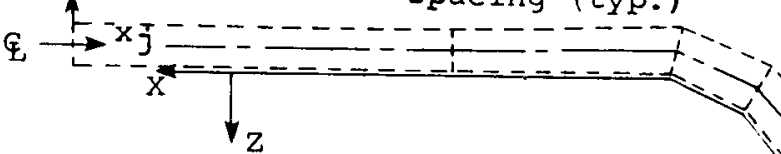

SIDE VIEW
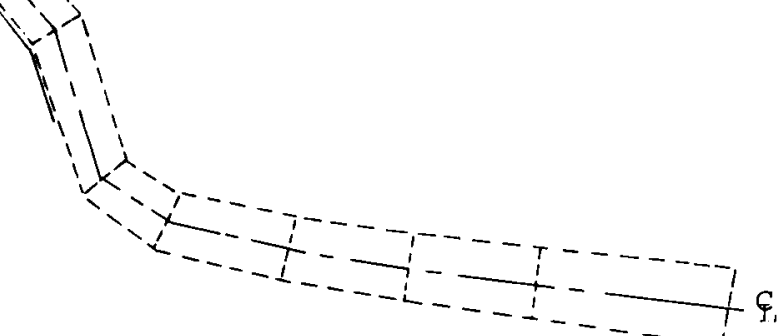

Figure 4.- Aspect ratio 6, rectangular jet model on a two-engine USB configuration, $\delta_{f}=72^{\circ}$. 

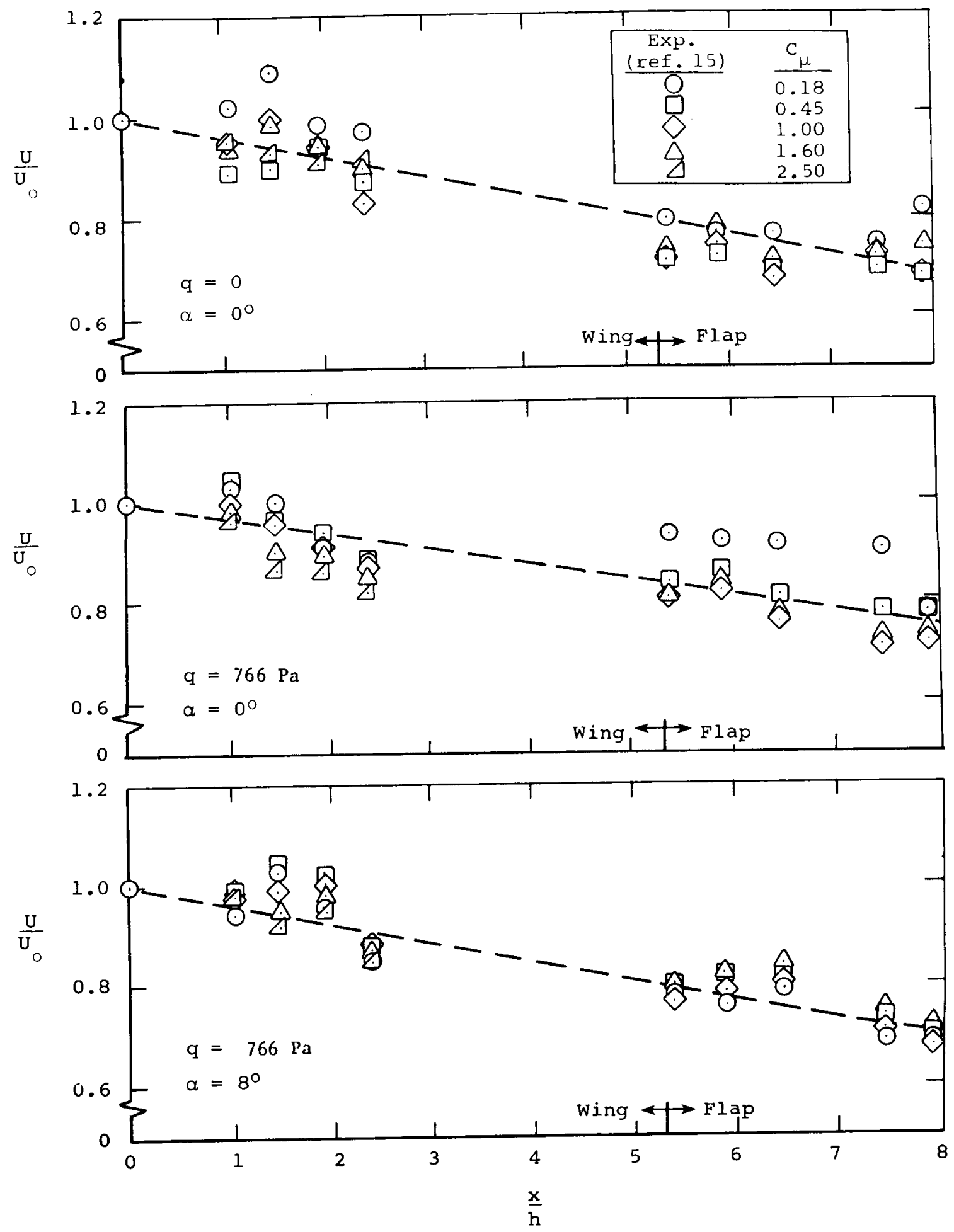

Figure 5.- Decay of the average velocity in an USB jet. 


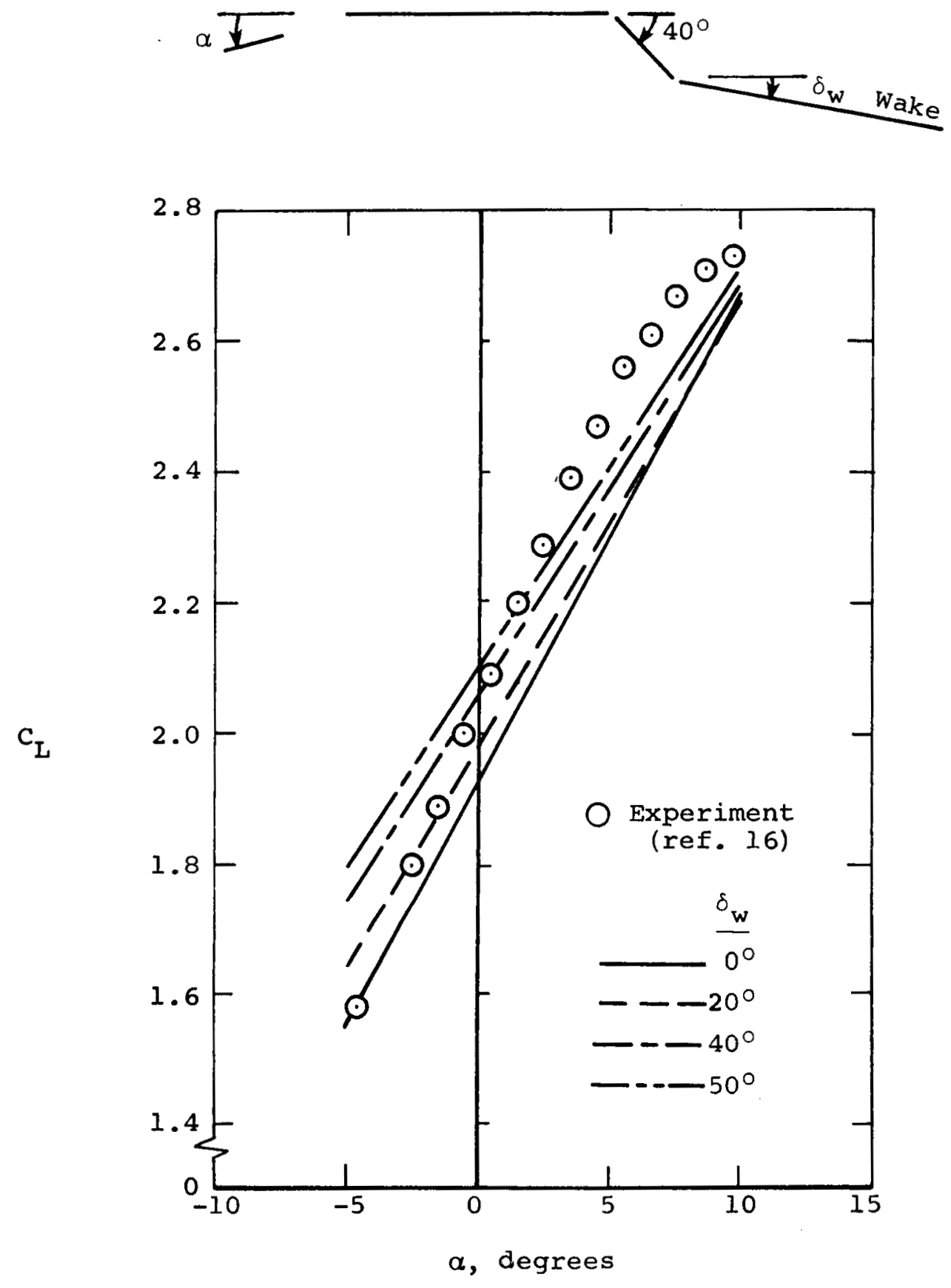

Figure 6.- Effect of wake deflection angle on the predicted lift coefficient on an aspect ratio 6, unswept, rectangular wing with a full span flap deflected 400 . 


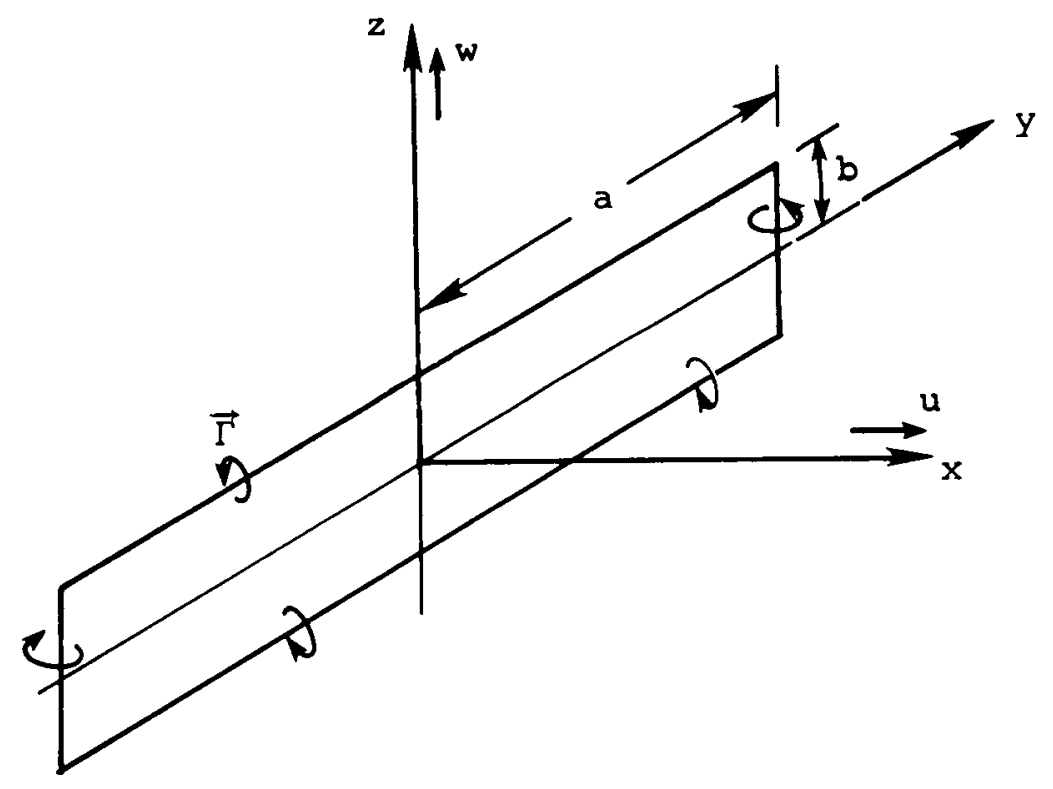

Figure 7.- Rectangular vortex ring. 


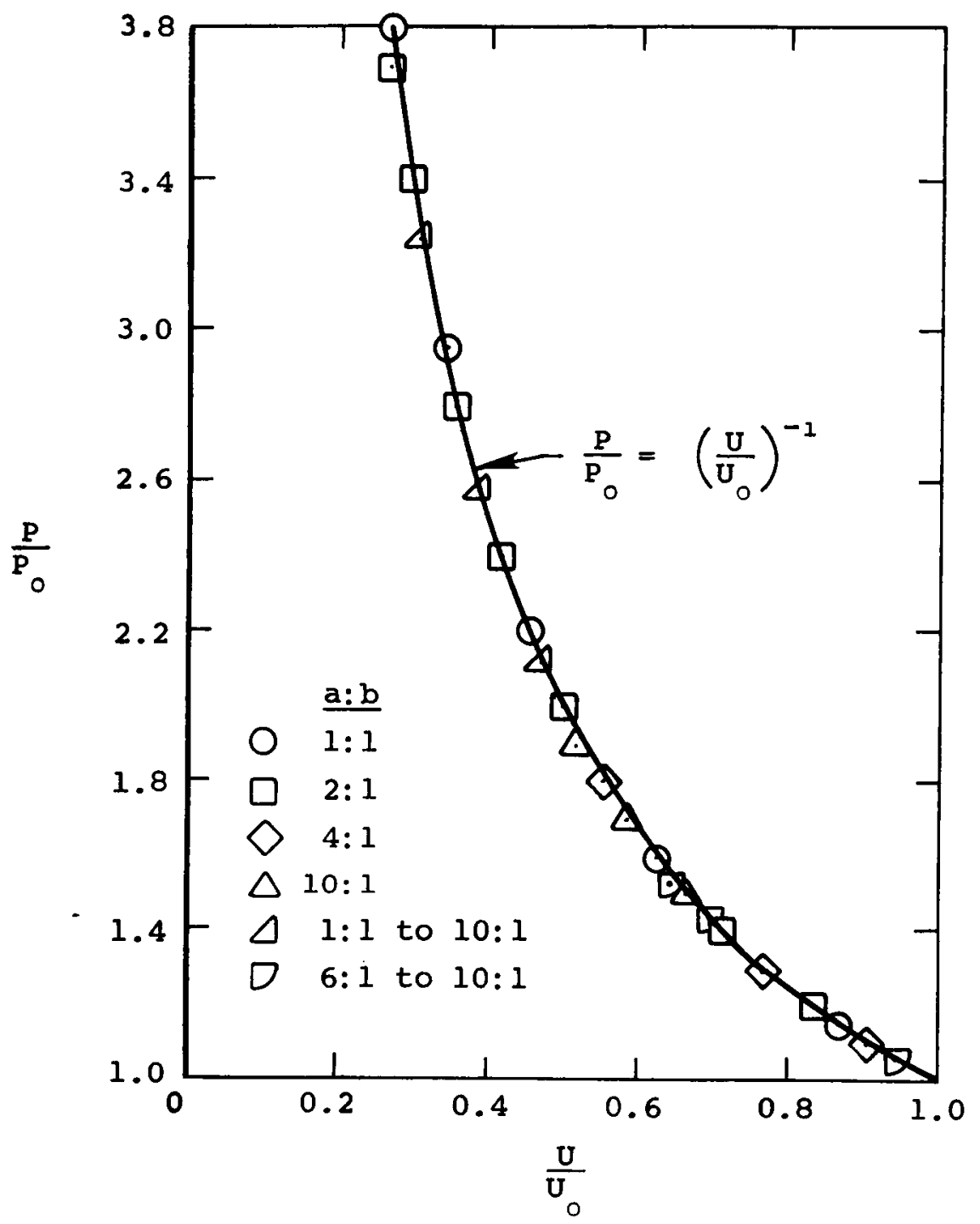

Figure 8.- Effect of jet expansion on the predicted axial velocity in a rectangular jet. 


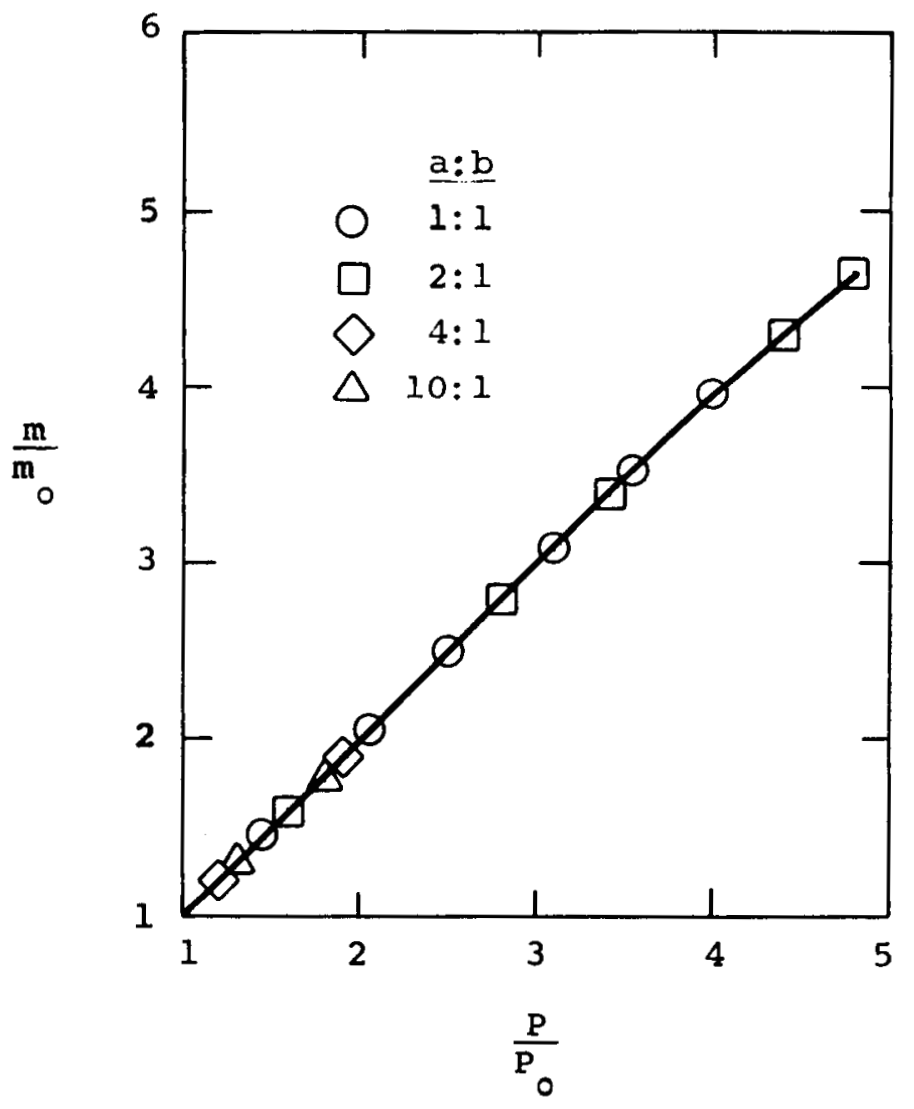

Figure 9.- Effect of expansion on the predicted mass flow in a constant aspect-ratio rectangular jet. 

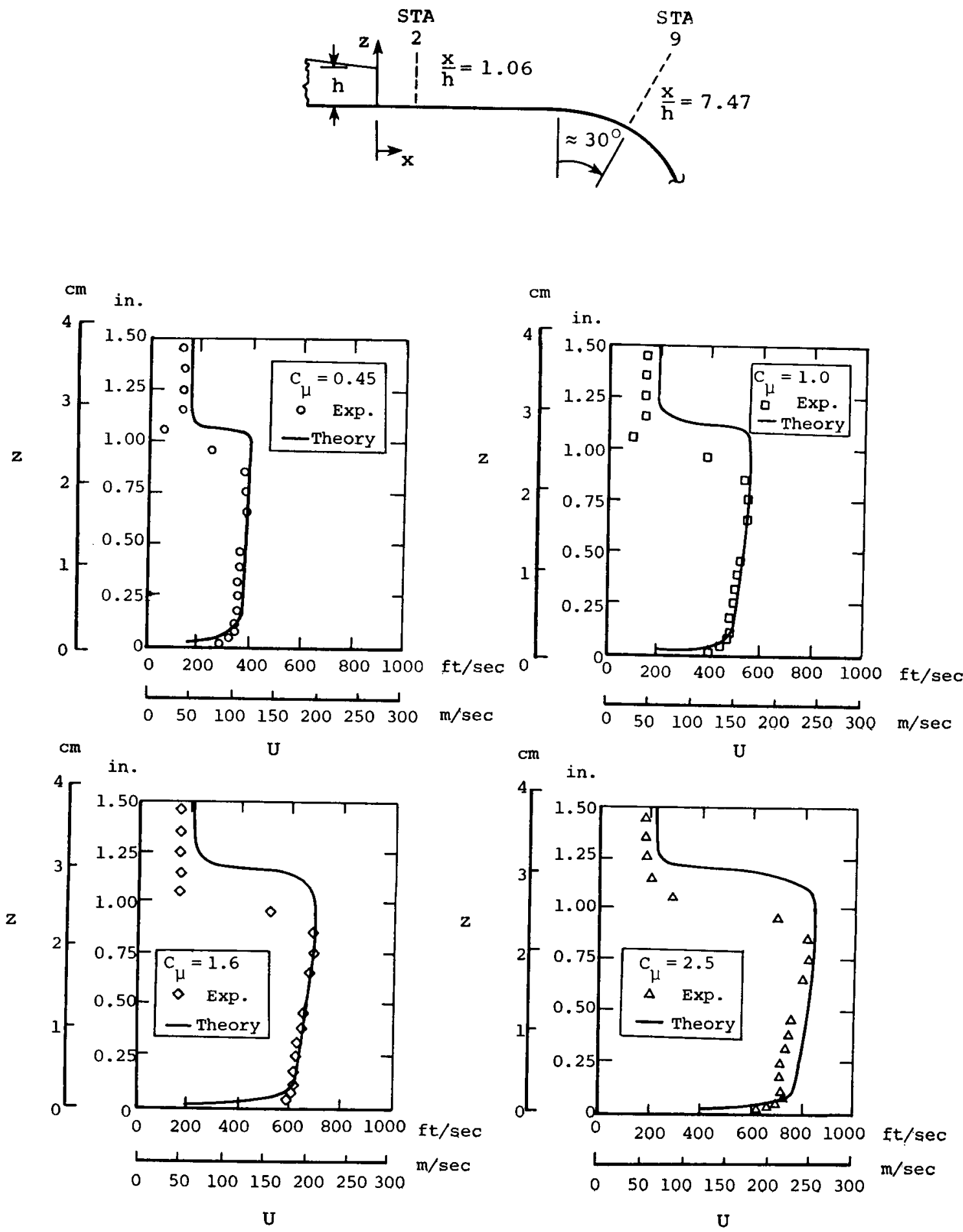

(a) Station $2, \mathrm{x} / \mathrm{h}=1.06$.

Figure 10.- Measured and predicted velocity profile on the upper surface of an USB configuration, reference 15 . 
in.
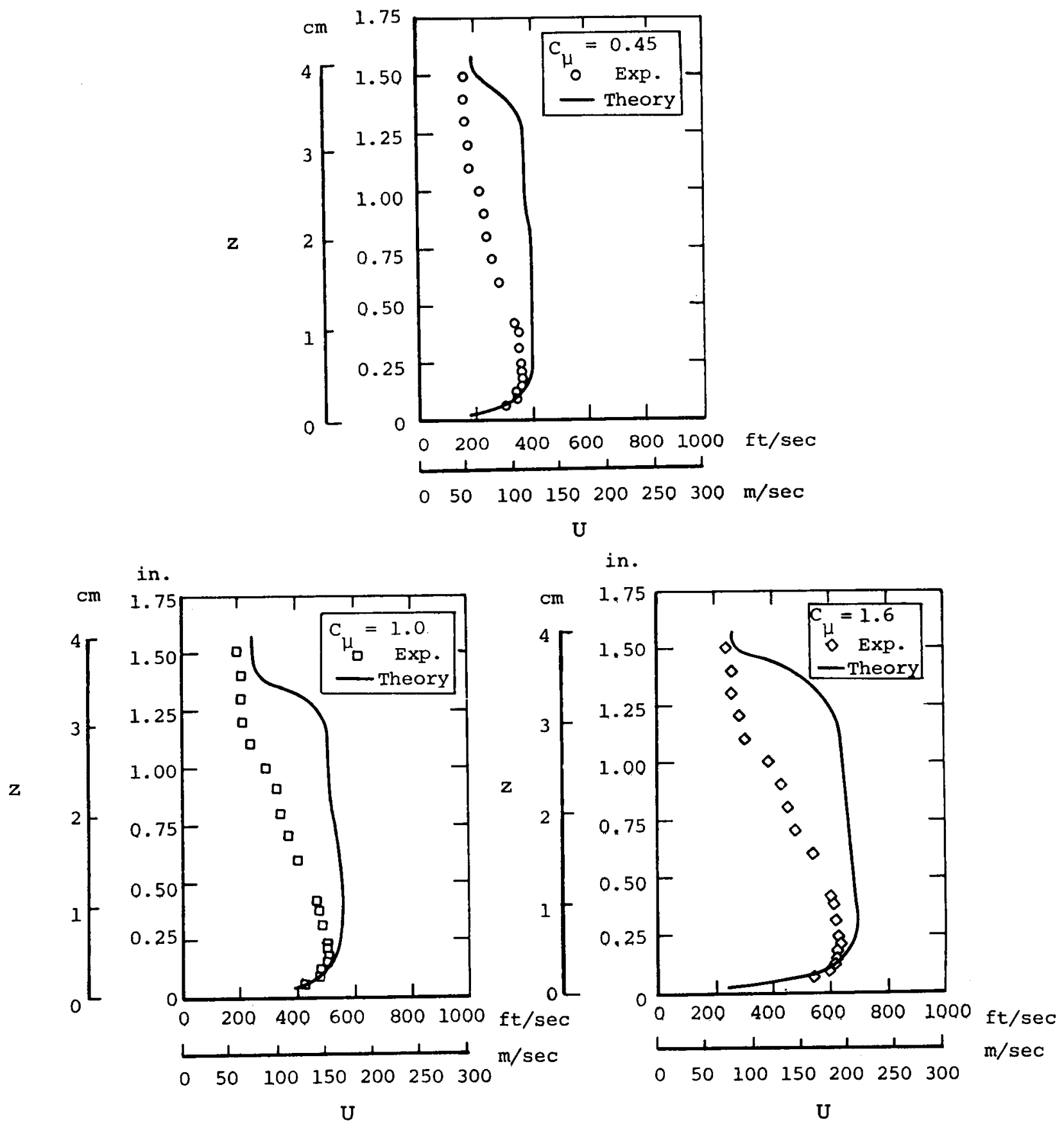

(b) Station $9, x / h=7.47$.

Figure 10.- Conciuded. 


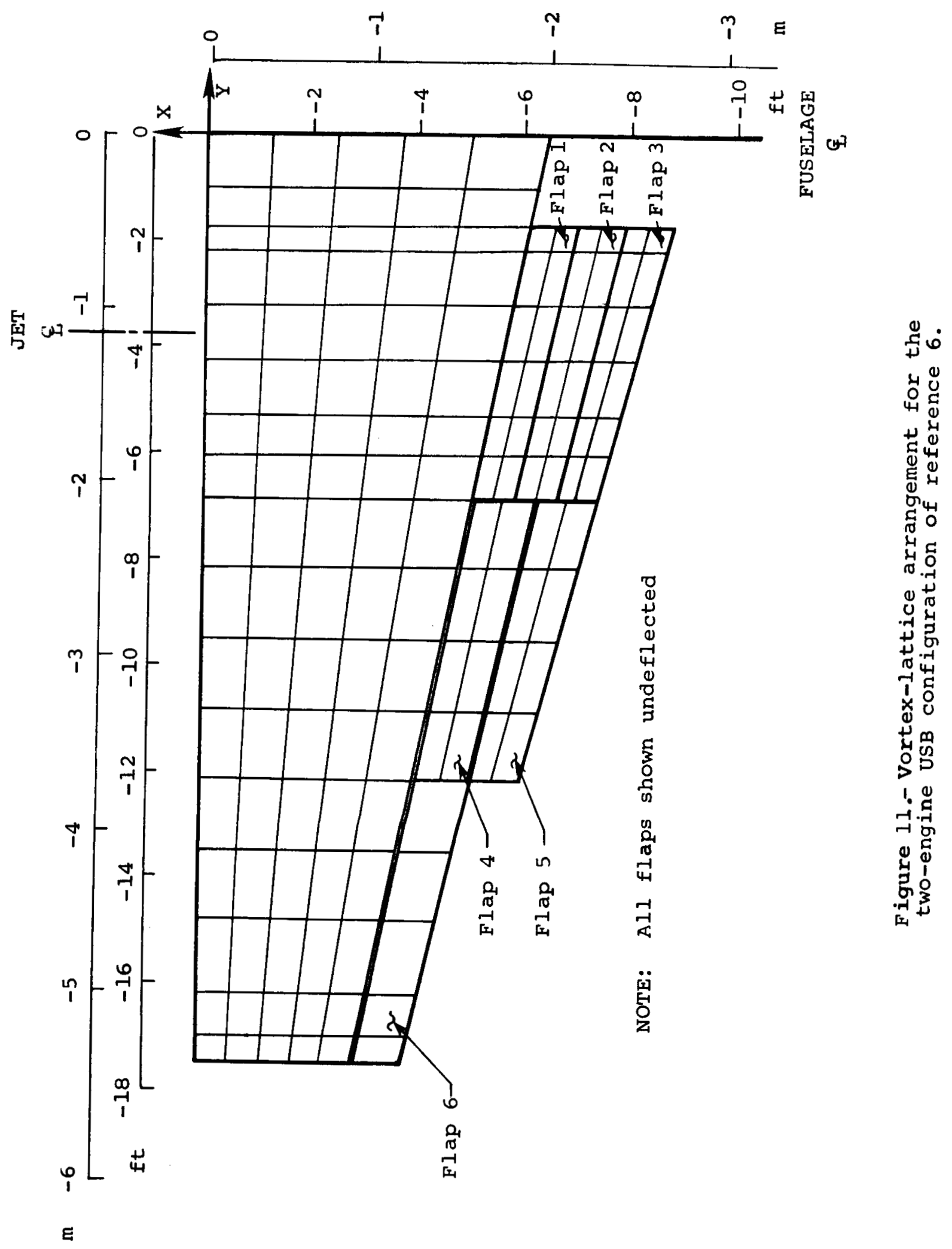




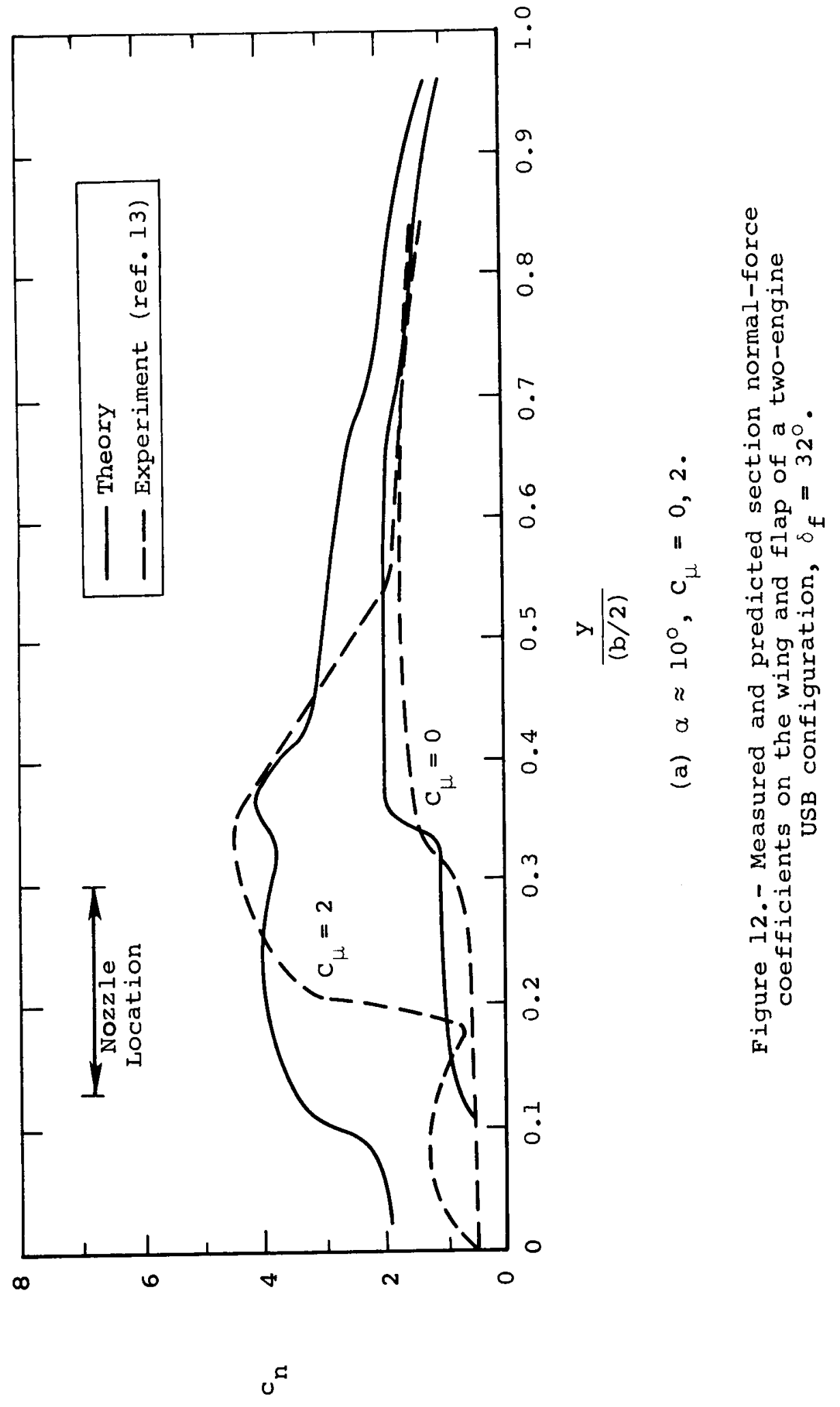




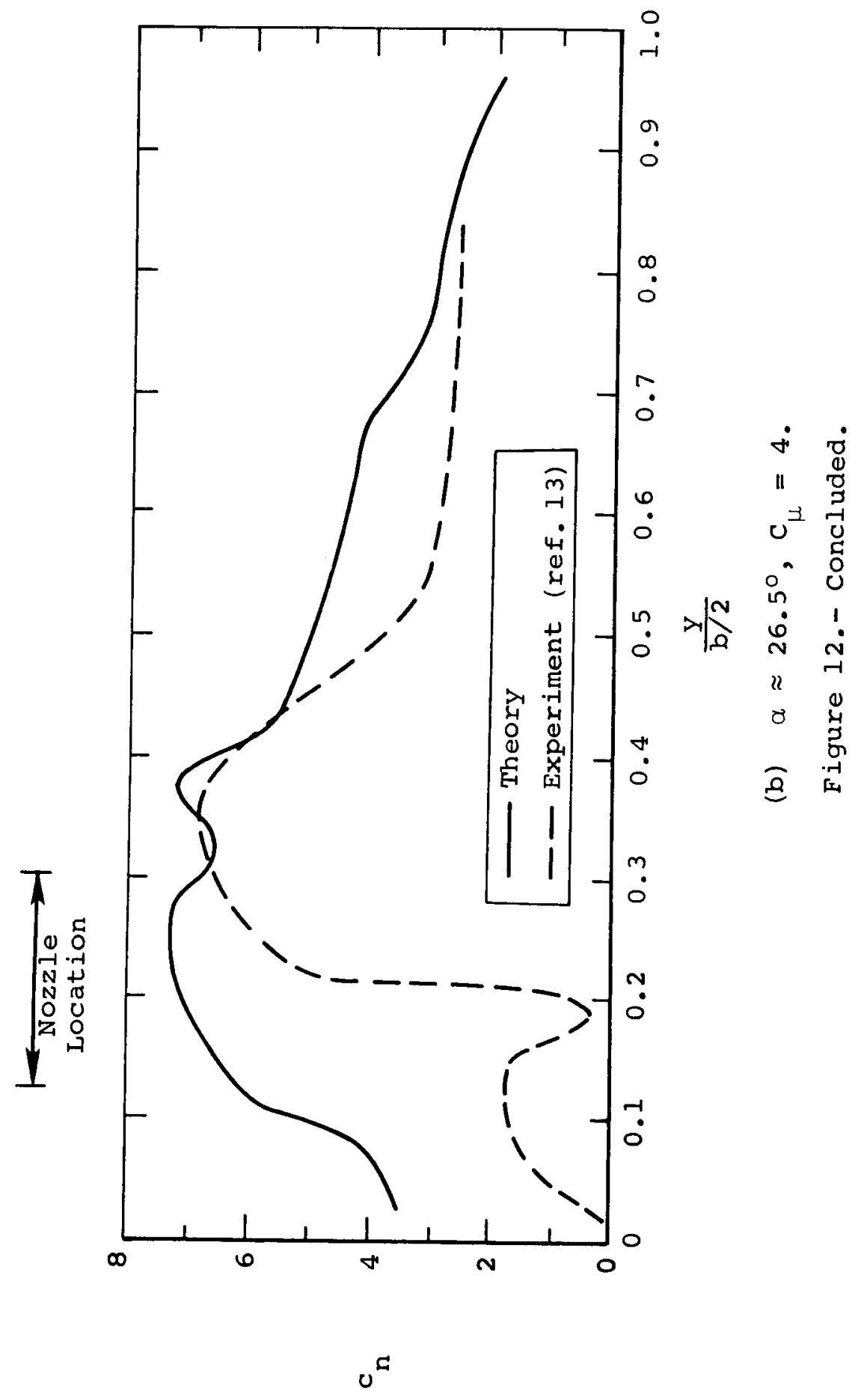



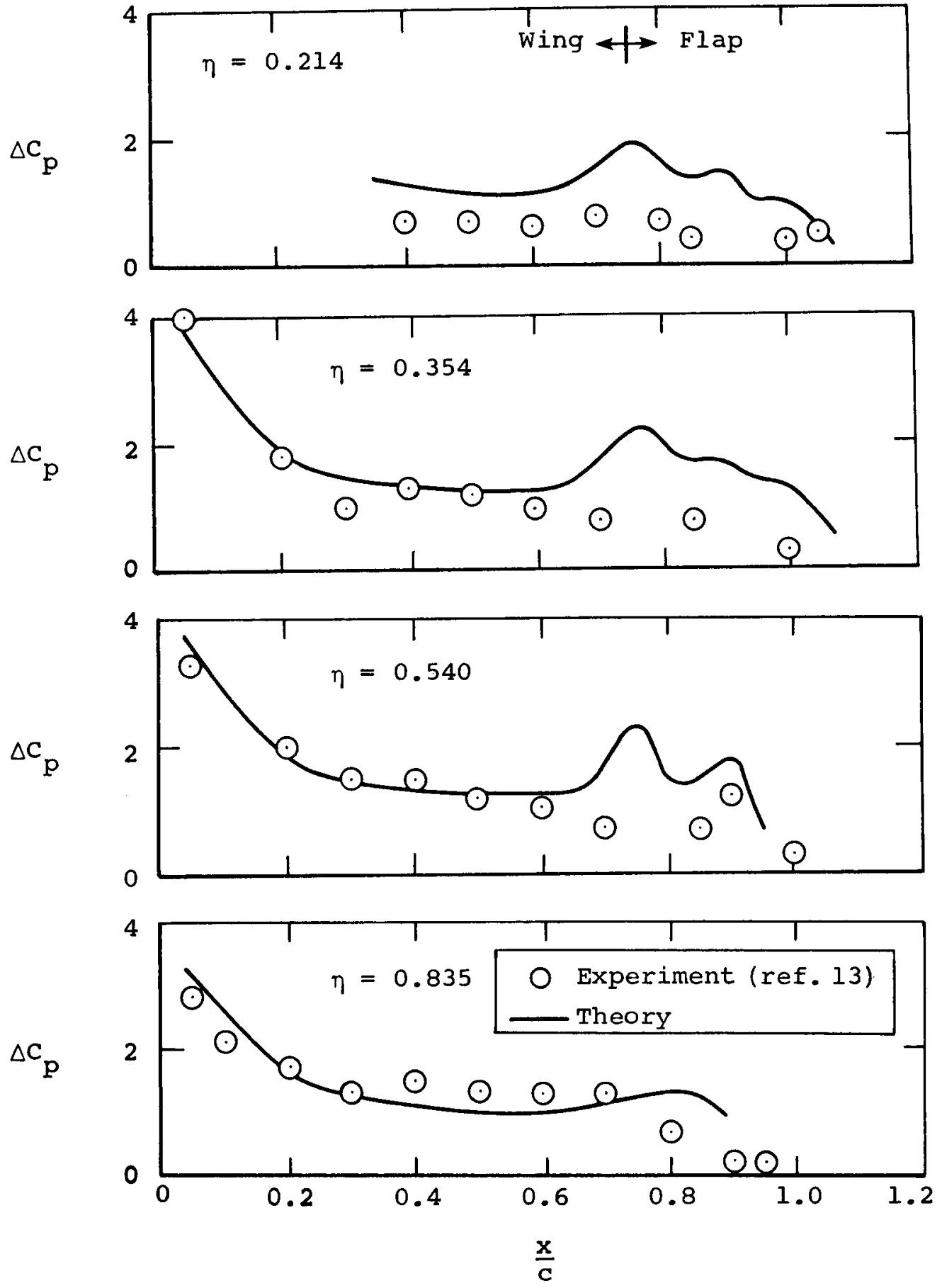

(a) $\alpha \approx 10^{\circ}, c_{\mu}=0$.

Figure 13.- Measured and predicted pressure distribution on a two-engine USB configuration, $\delta_{f}=32^{\circ}$. 

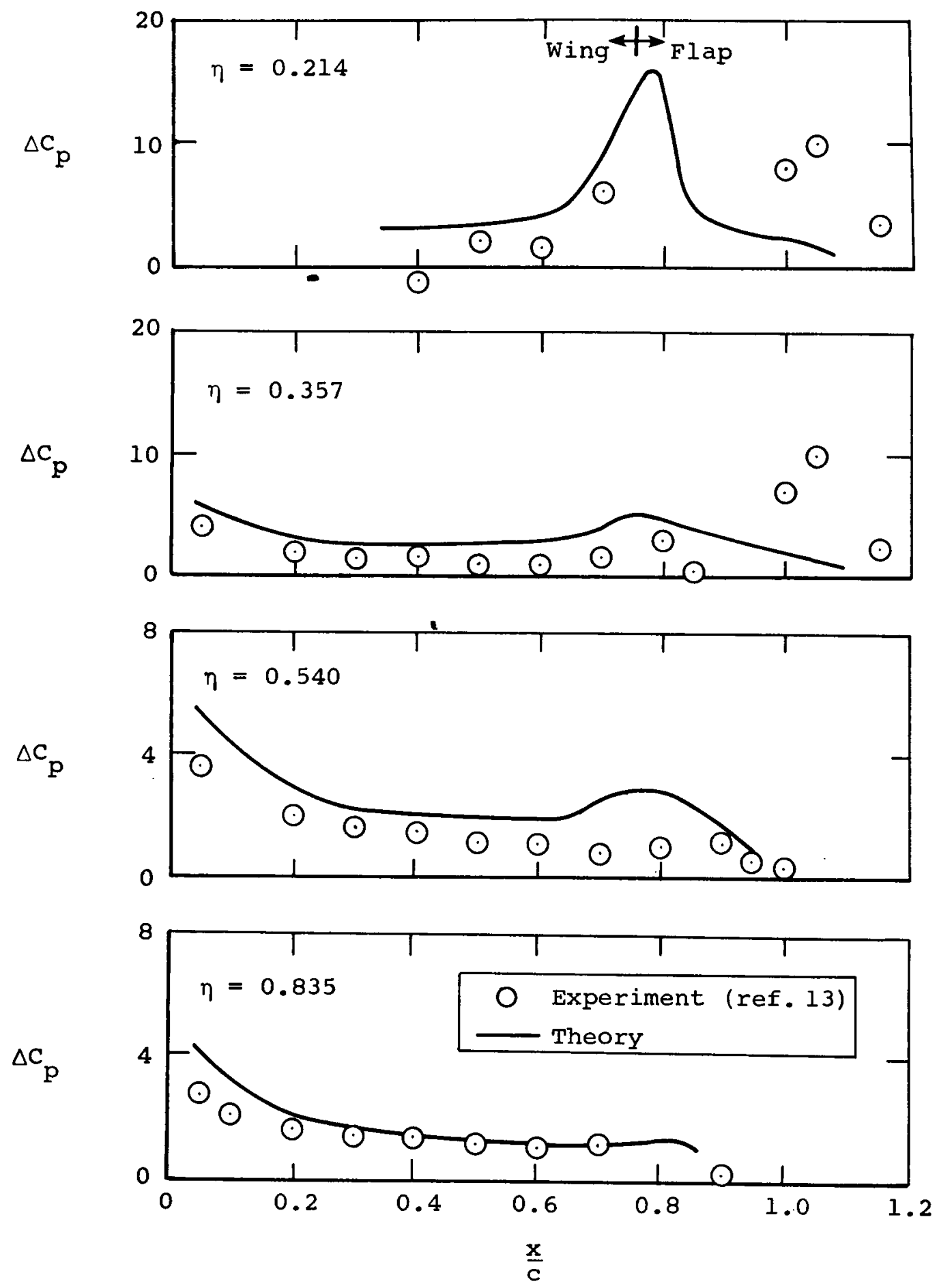

(b) $\alpha \approx 10^{\circ}, c_{\mu} \approx 2$.

Figure 13.- Concluded. 


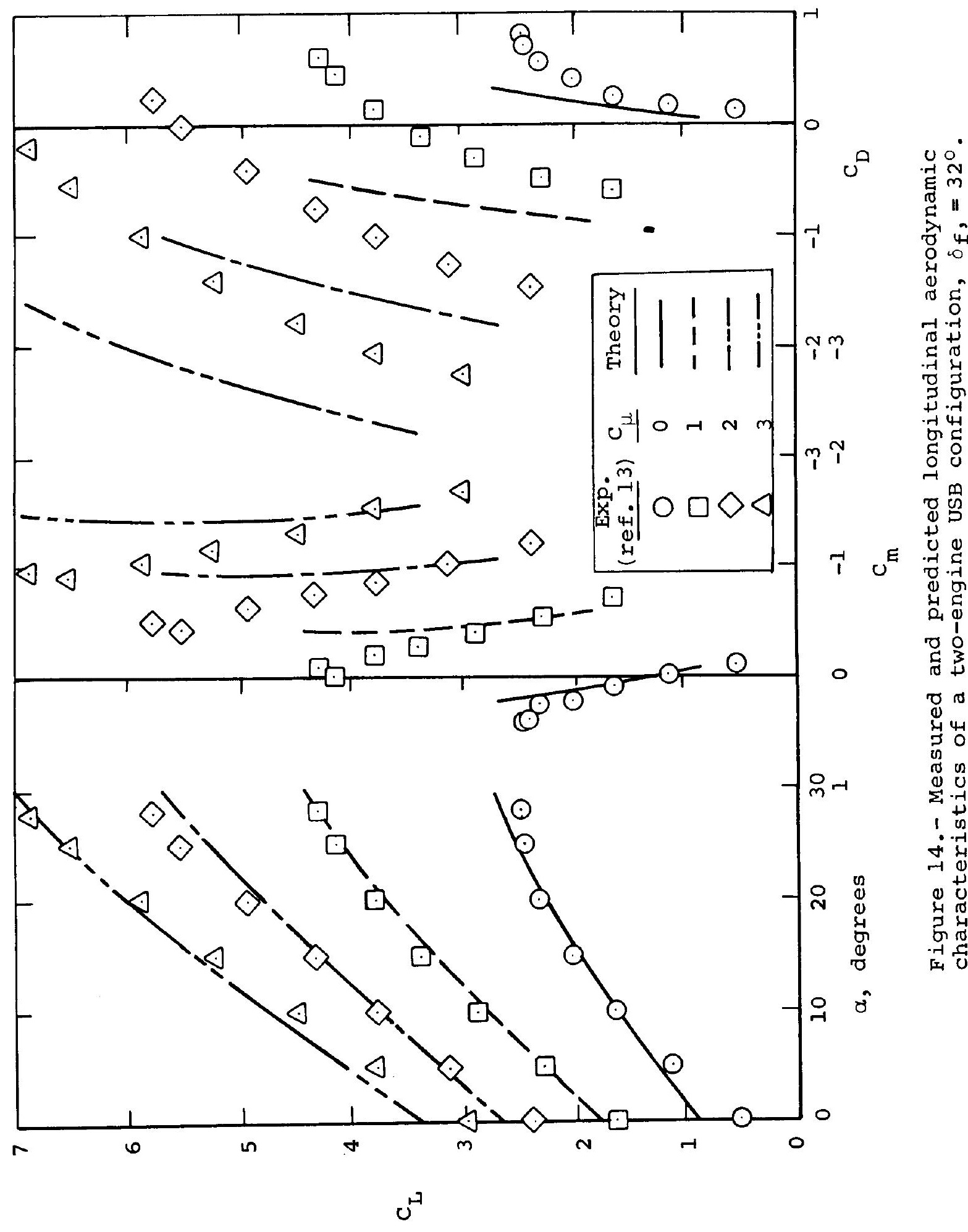




$$
\Delta C_{L}=\left.C_{L}\right|_{C_{\mu}>0}-\left.C_{L}\right|_{C_{\mu}}=0
$$

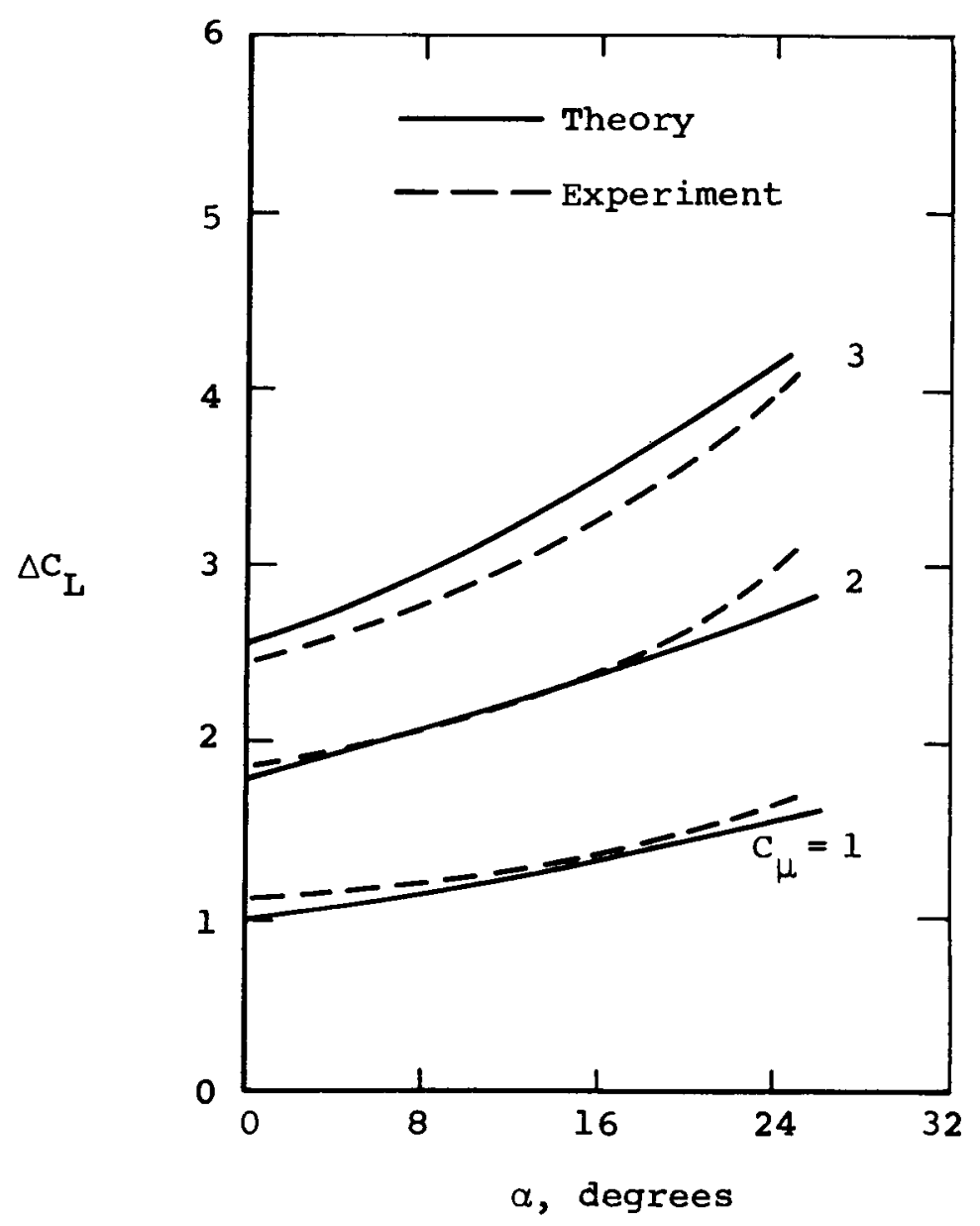

Figure 15.- Measured and predicted jet-induced lift on a two-engine USB configuration, $\delta_{f}=32^{\circ}$. 

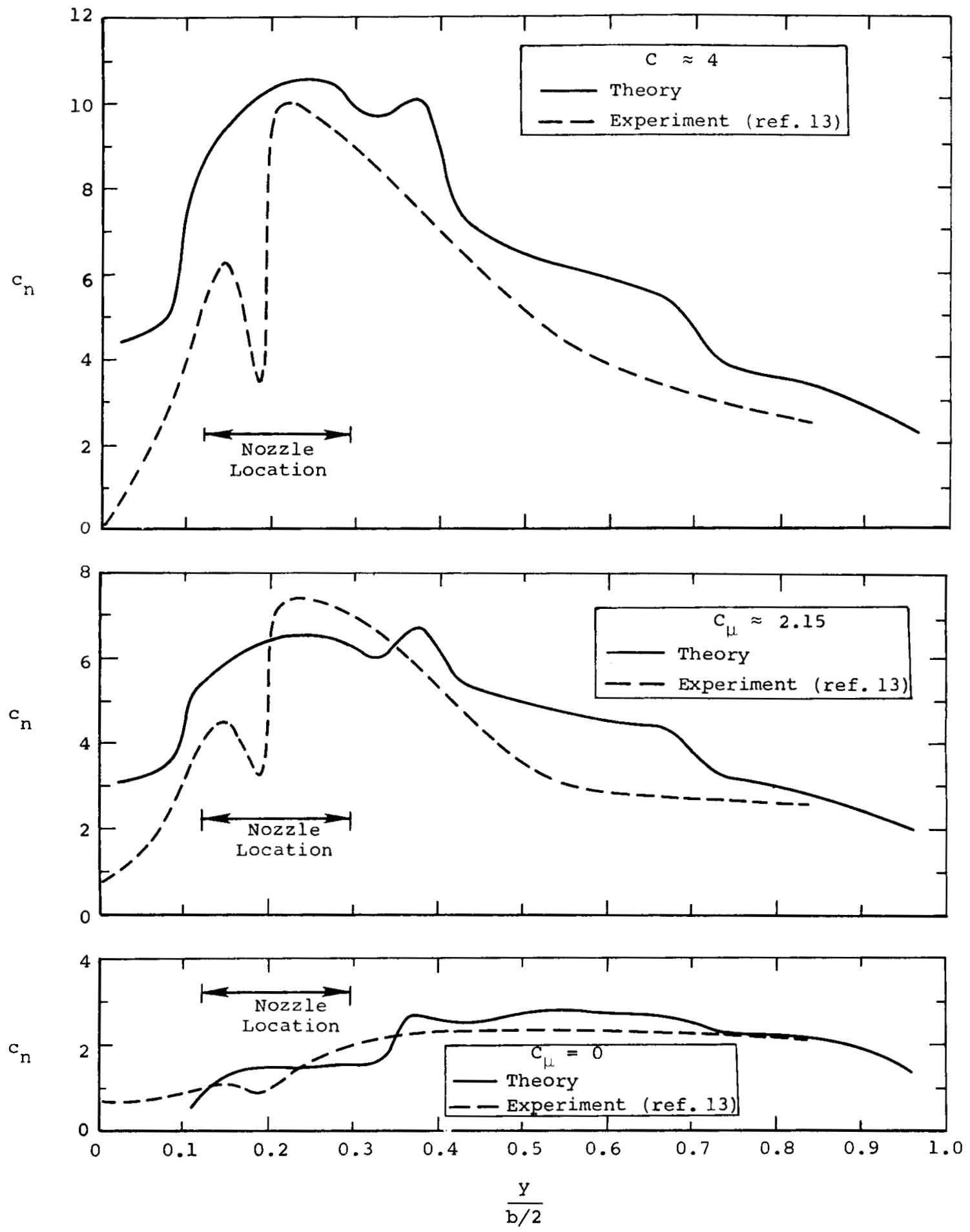

(a) $\alpha \approx 10^{\circ}$.

Figure 16.- Measured and predicted section normal-force coefficients on the wing and flap of a two-engine USB configuration, $\delta_{f}=72^{\circ}$. 

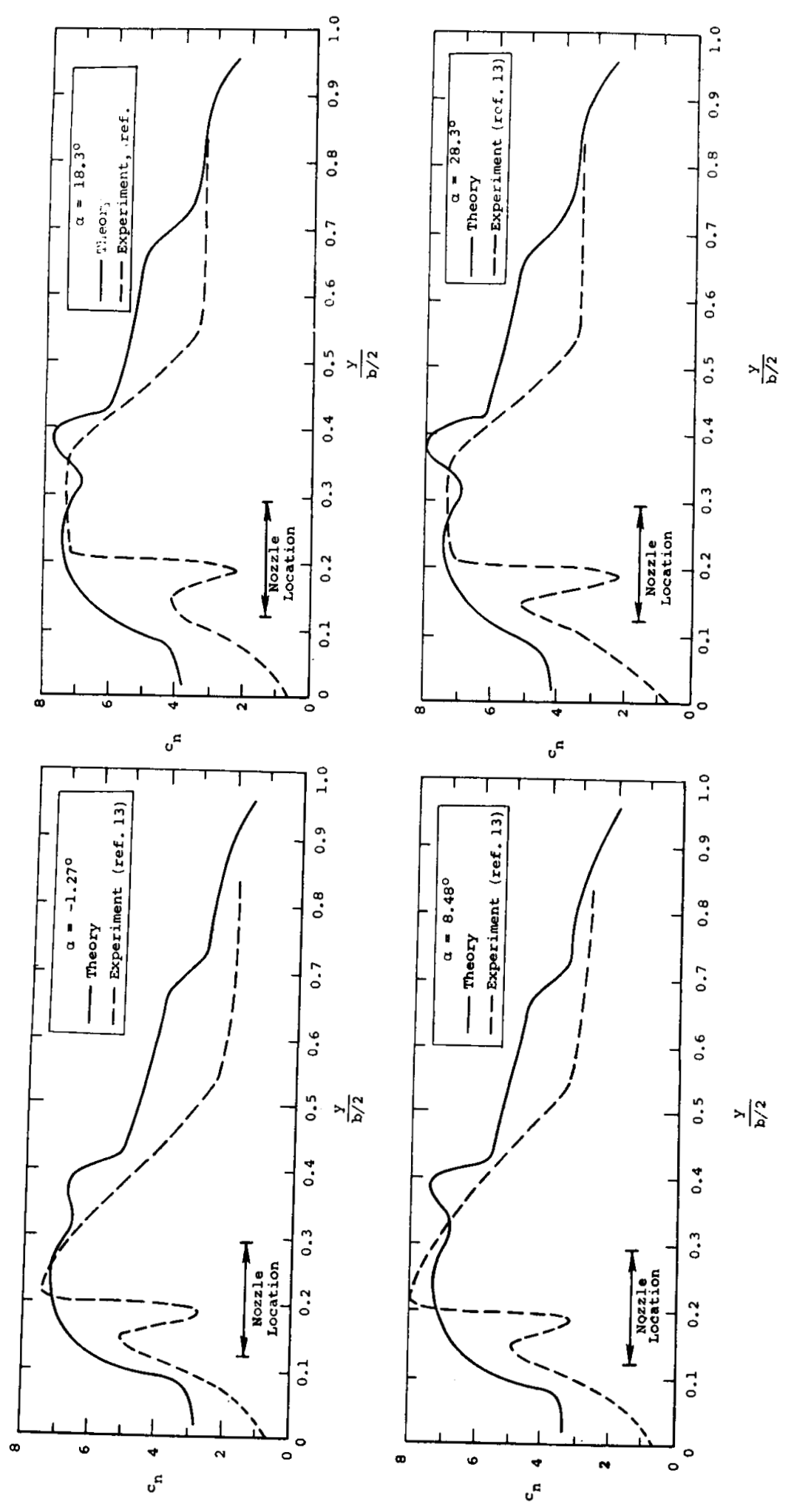

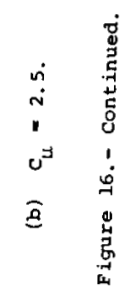




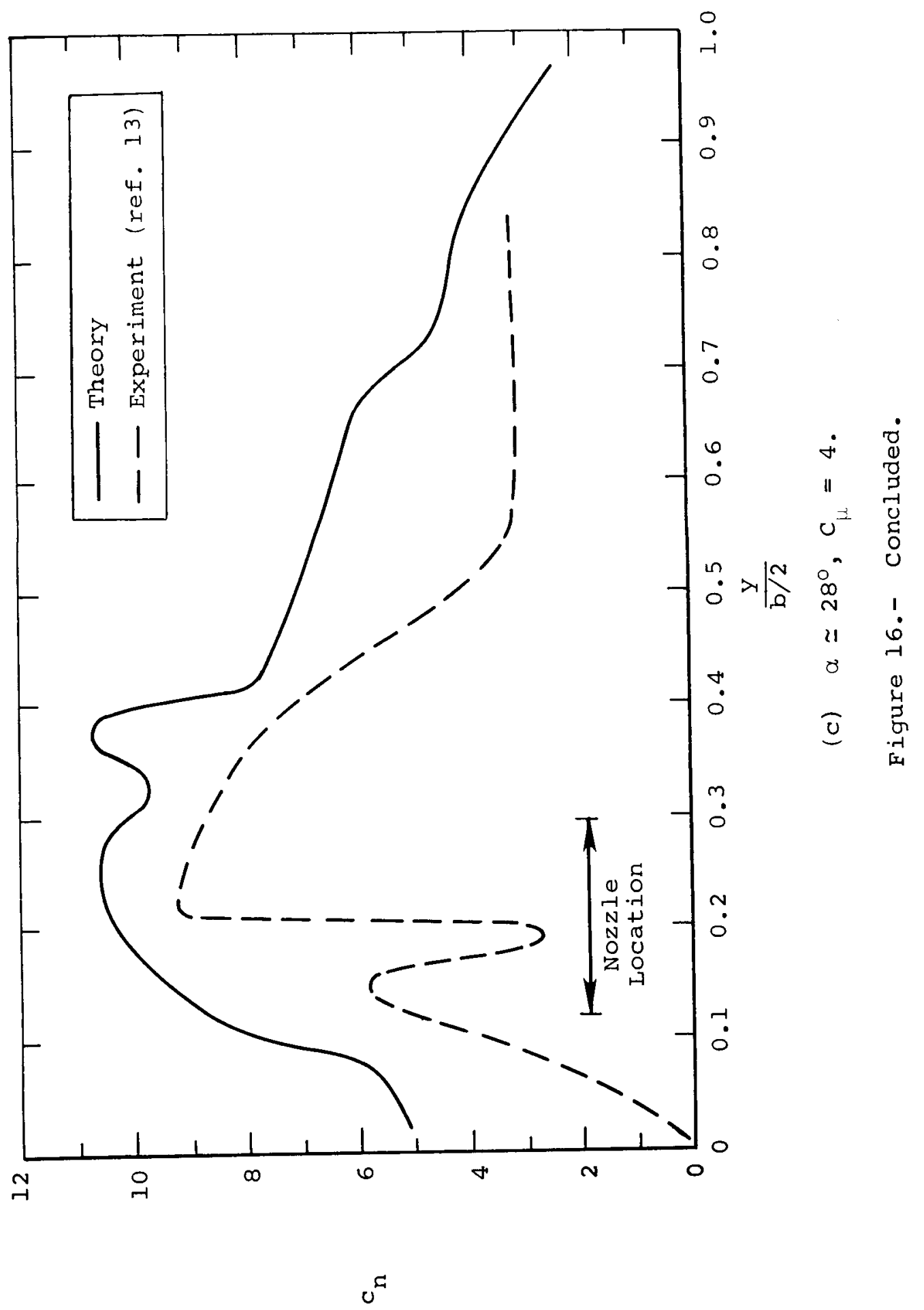



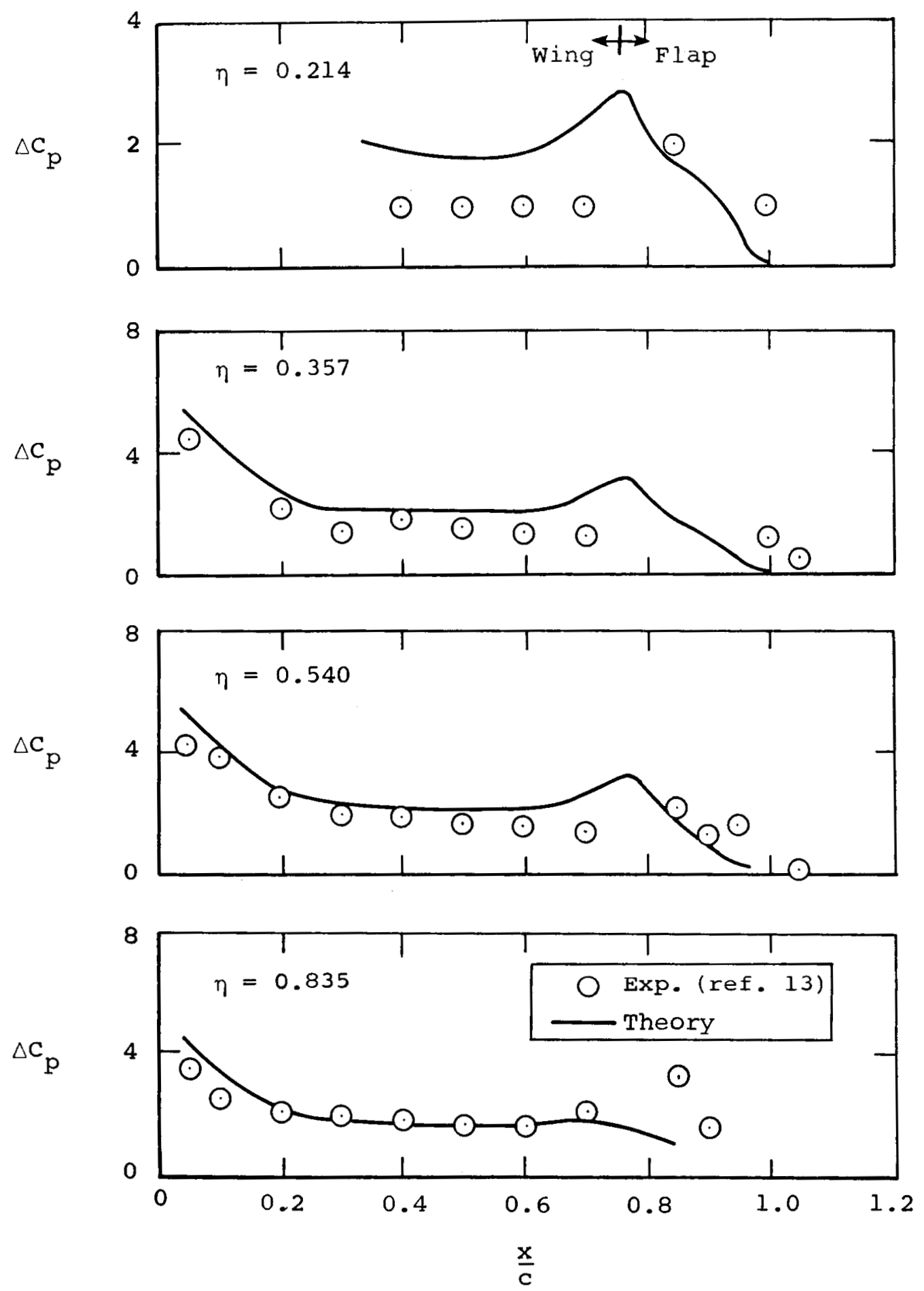

(a) $\alpha \simeq 10^{\circ}, \mathrm{c}_{\mu}=0$.

Figure 17.- Measured and predicted pressure distribution on a two-engine USB configuration, $\delta_{f}=72^{\circ}$. 

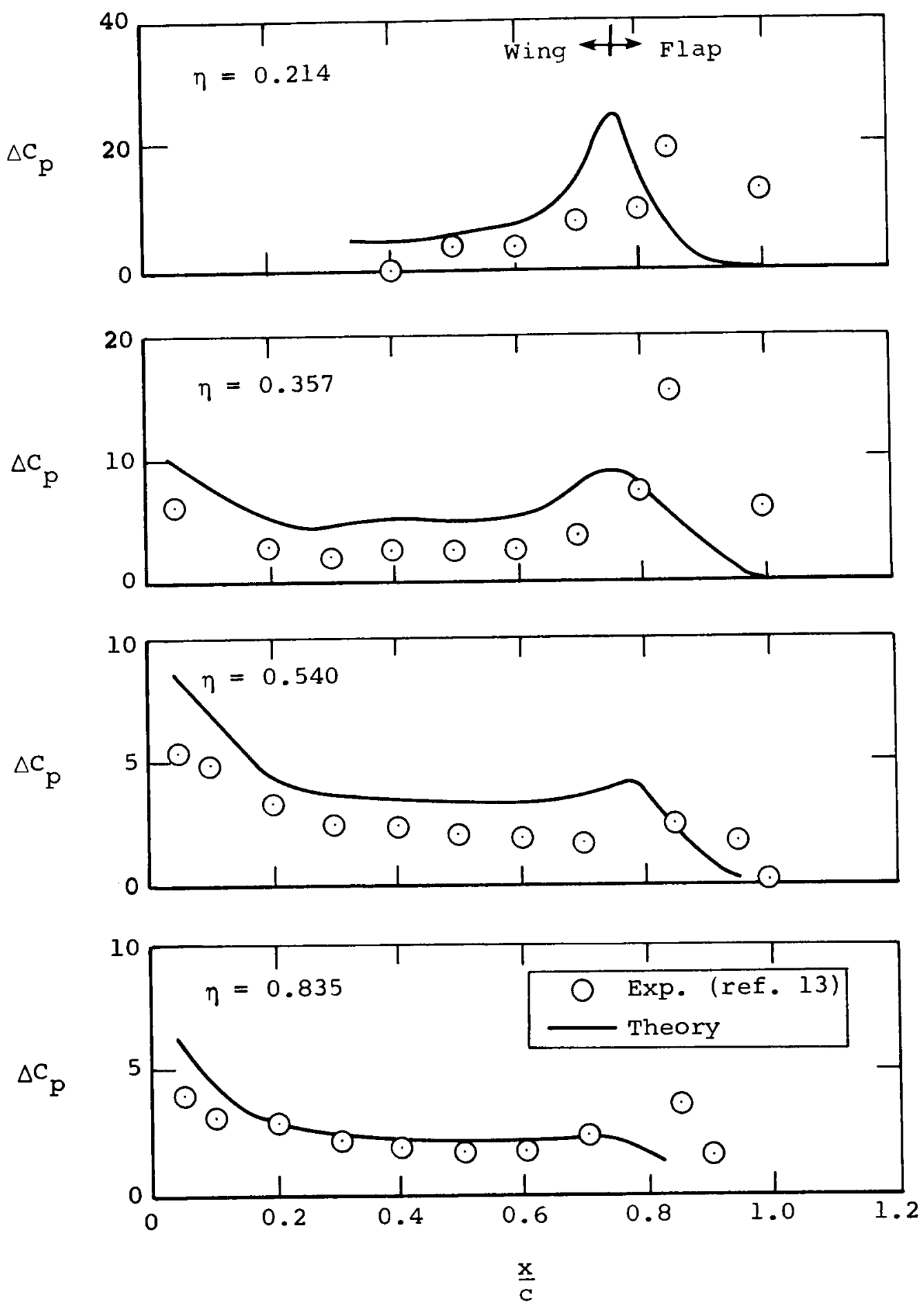

(b) $\alpha \simeq 10^{\circ}, c_{\mu}=2$.

Figure 17.- Continued. 

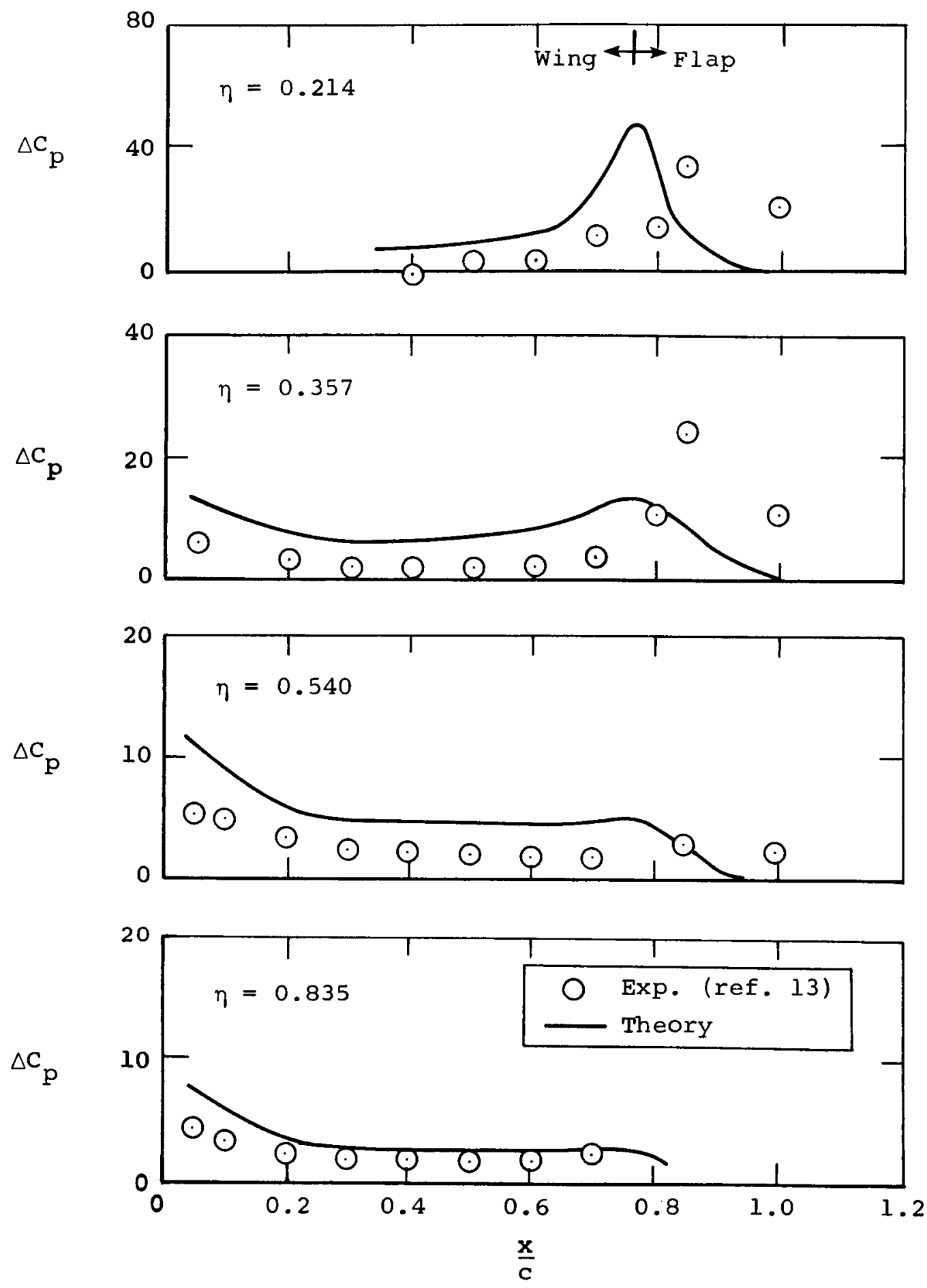

(c) $a \simeq 10^{\circ}, c_{\mu}=4$.

Figure 17.- Concluded. 


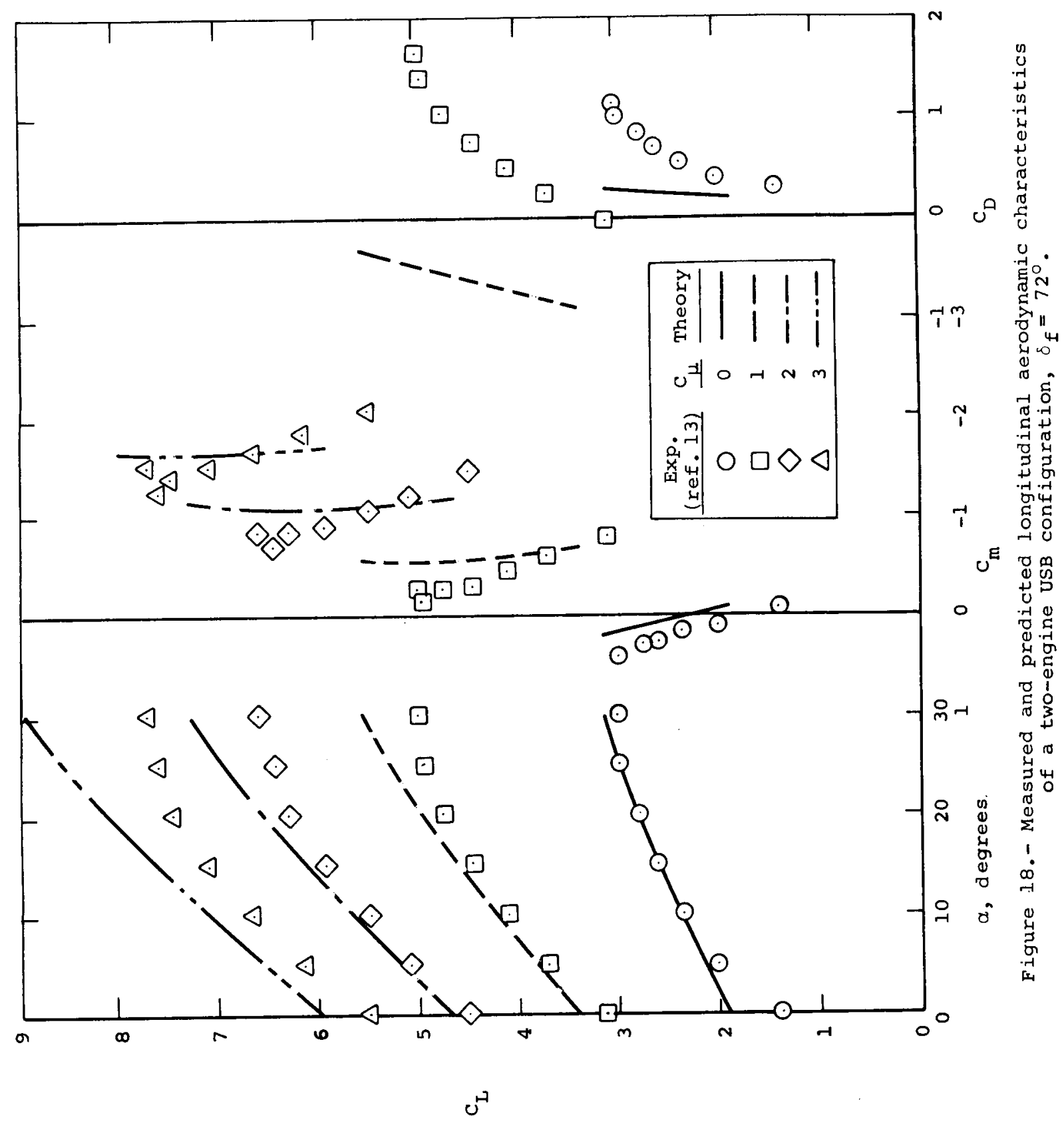




$$
\begin{gathered}
- \text { Theory } \\
- \text { Experiment } \\
\Delta C_{L}=\left.C_{L}\right|_{C_{\mu}>0}-\left.C_{L}\right|_{C_{\mu}}=0
\end{gathered}
$$

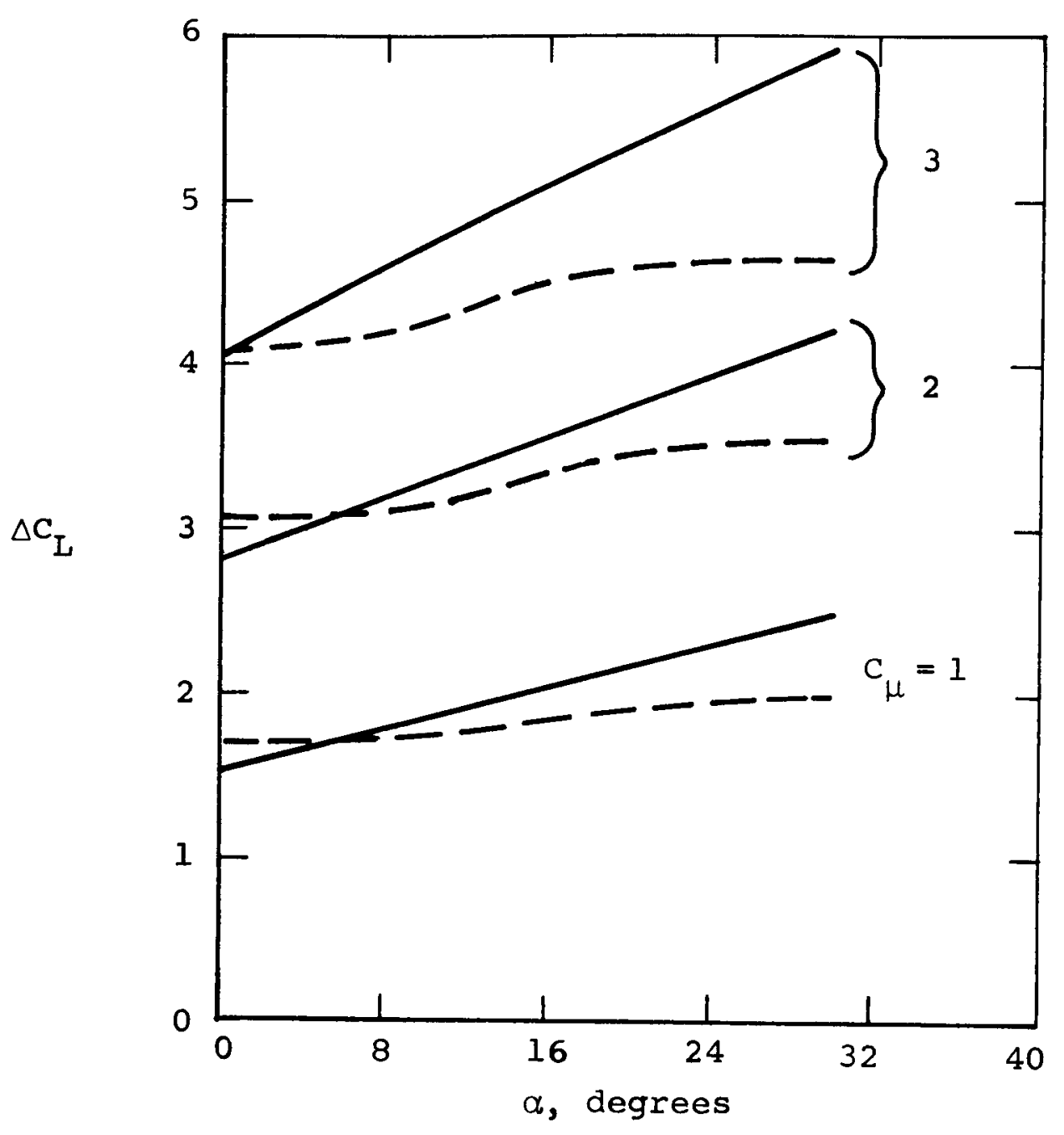

Figure 19.- Measured and predicted jet-induced lift on a two-engine USB configuration, $\delta_{f}=72^{\circ}$. 


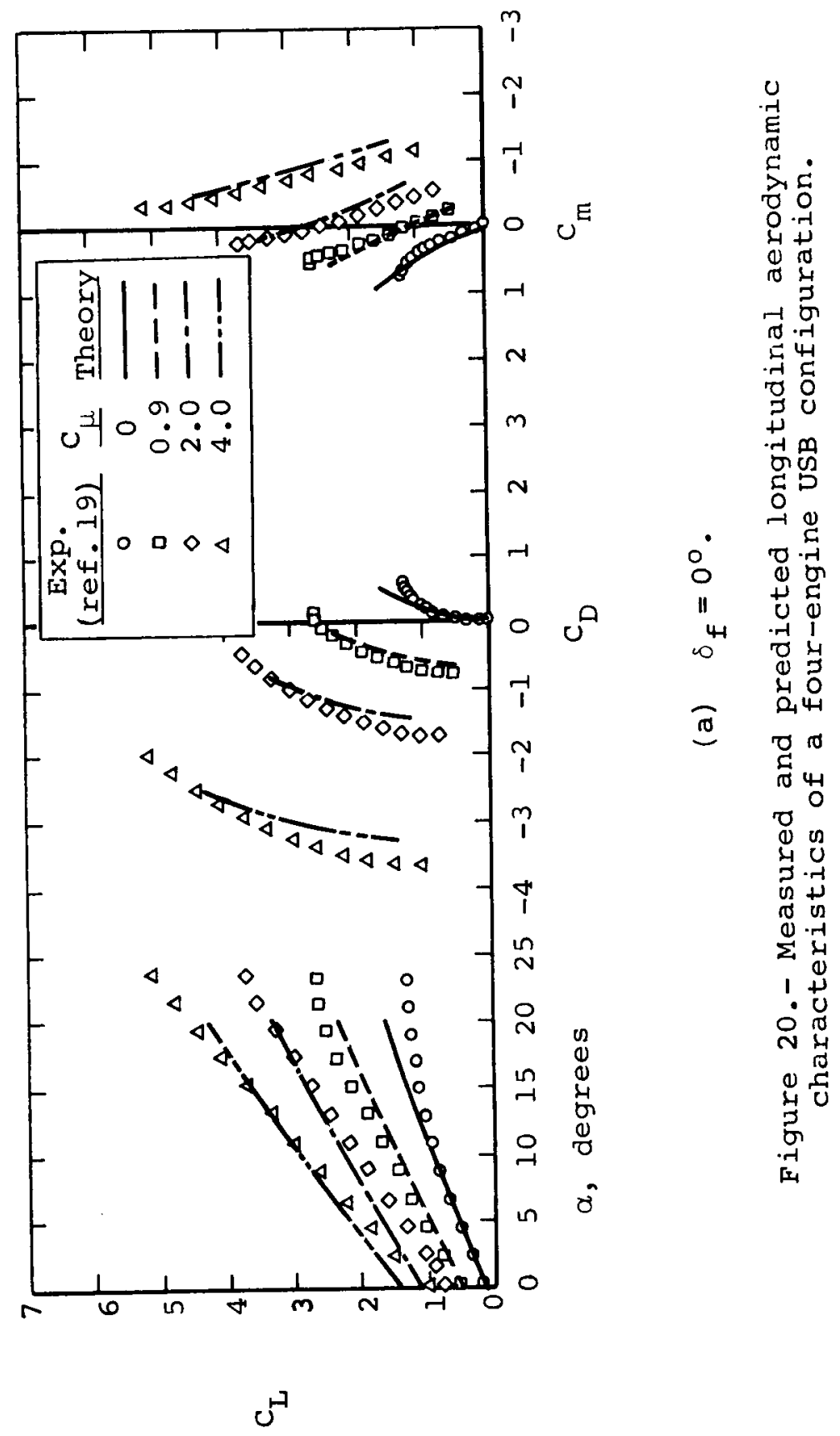




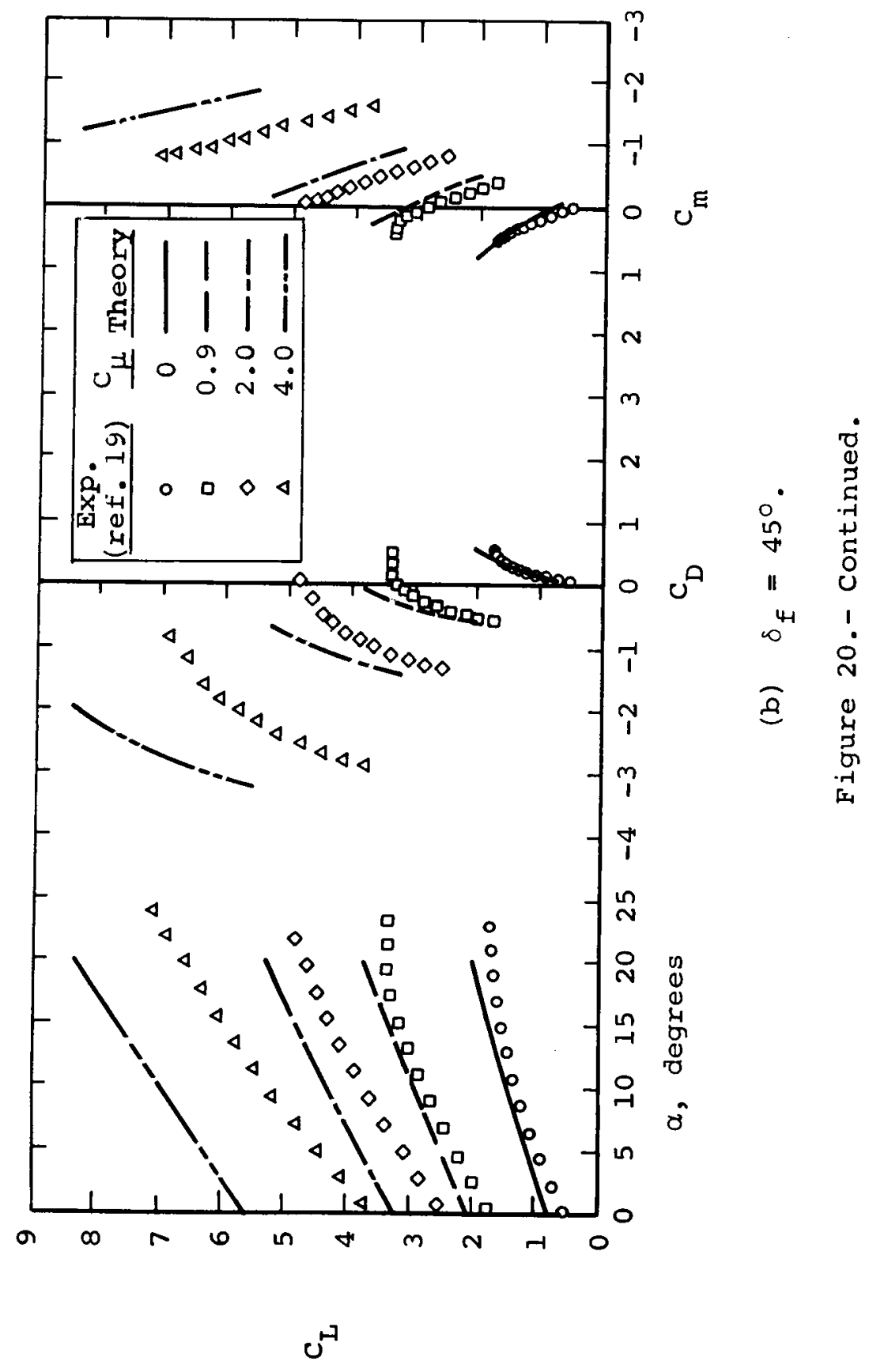

75 


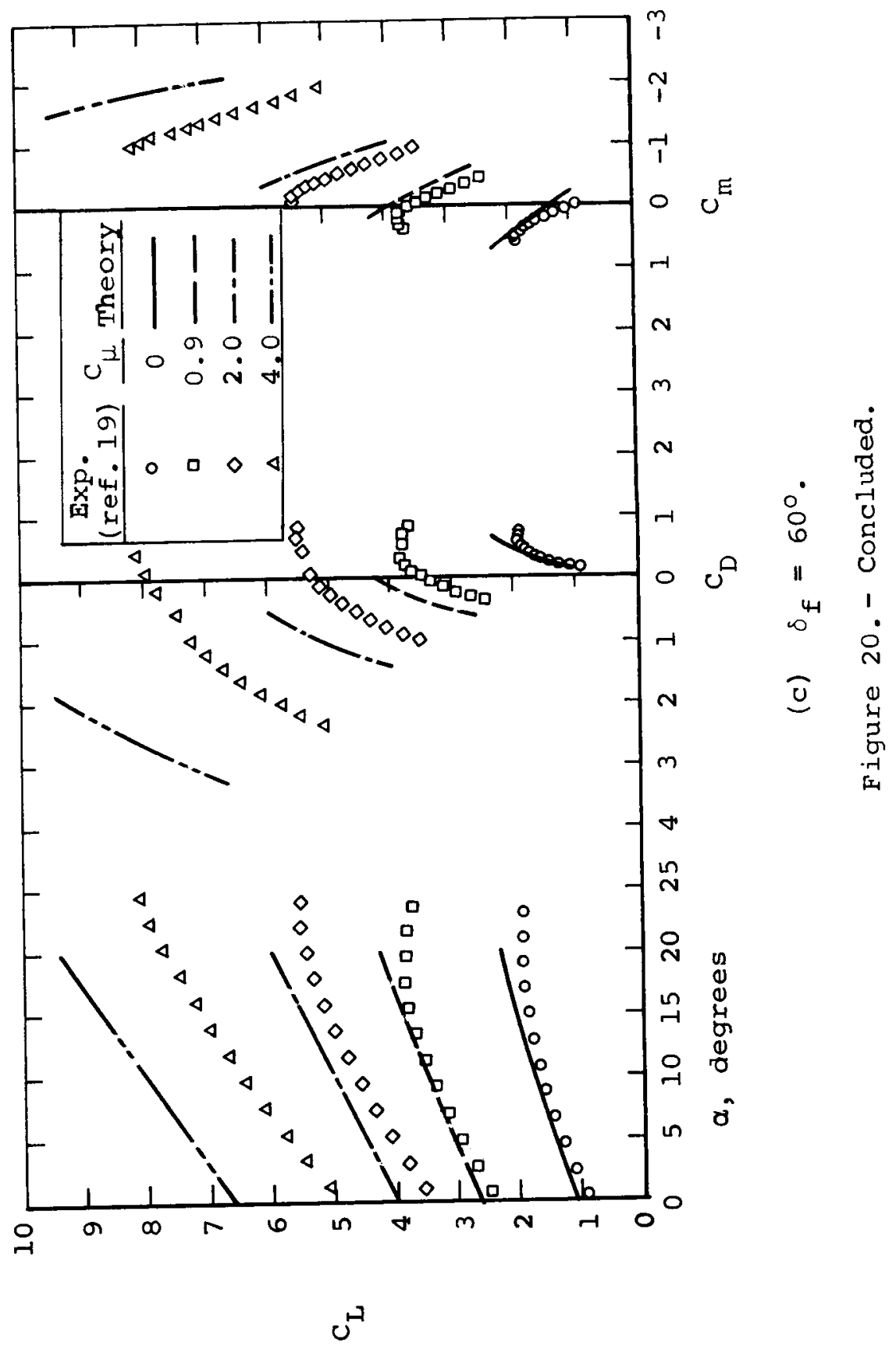




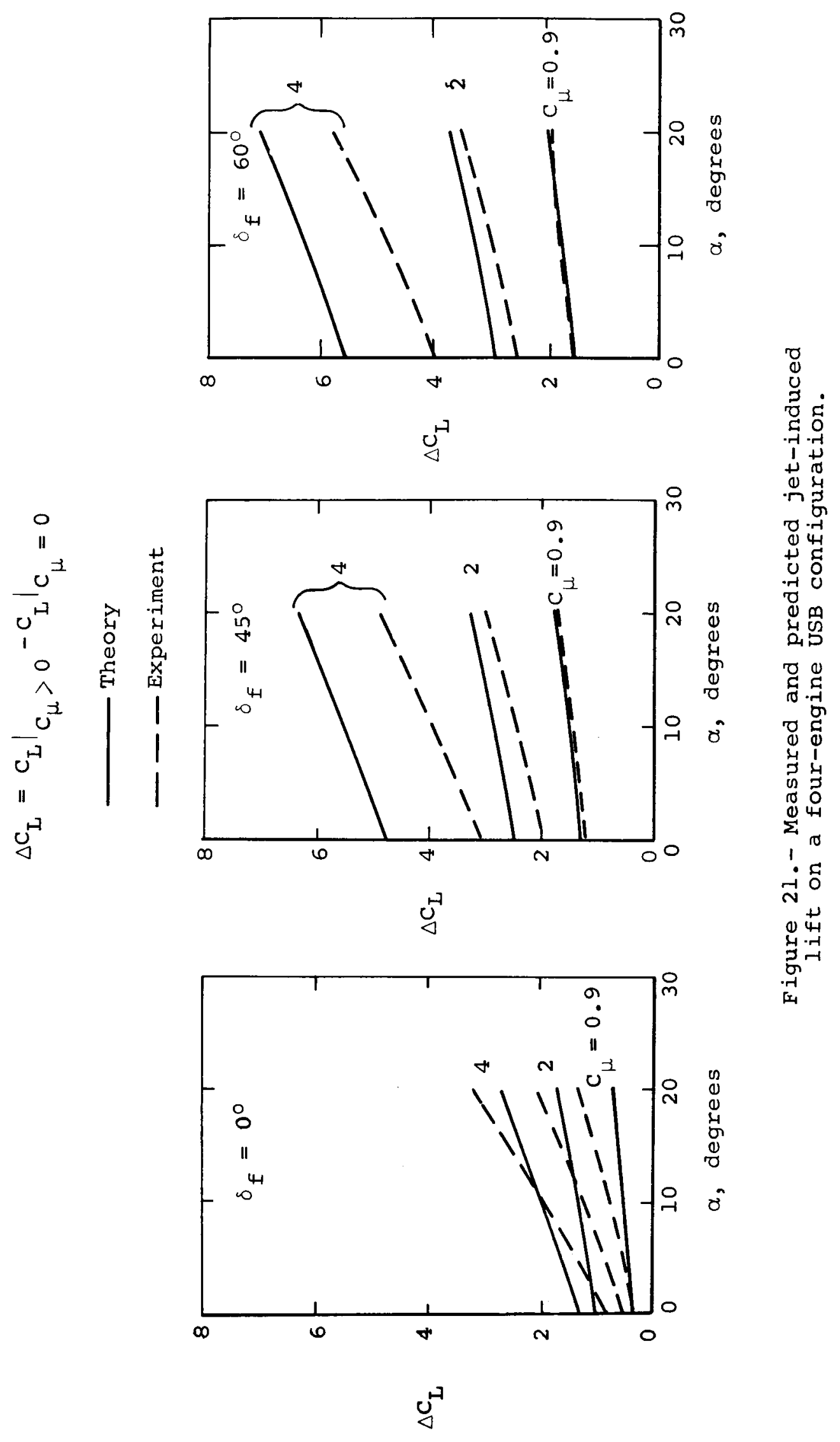




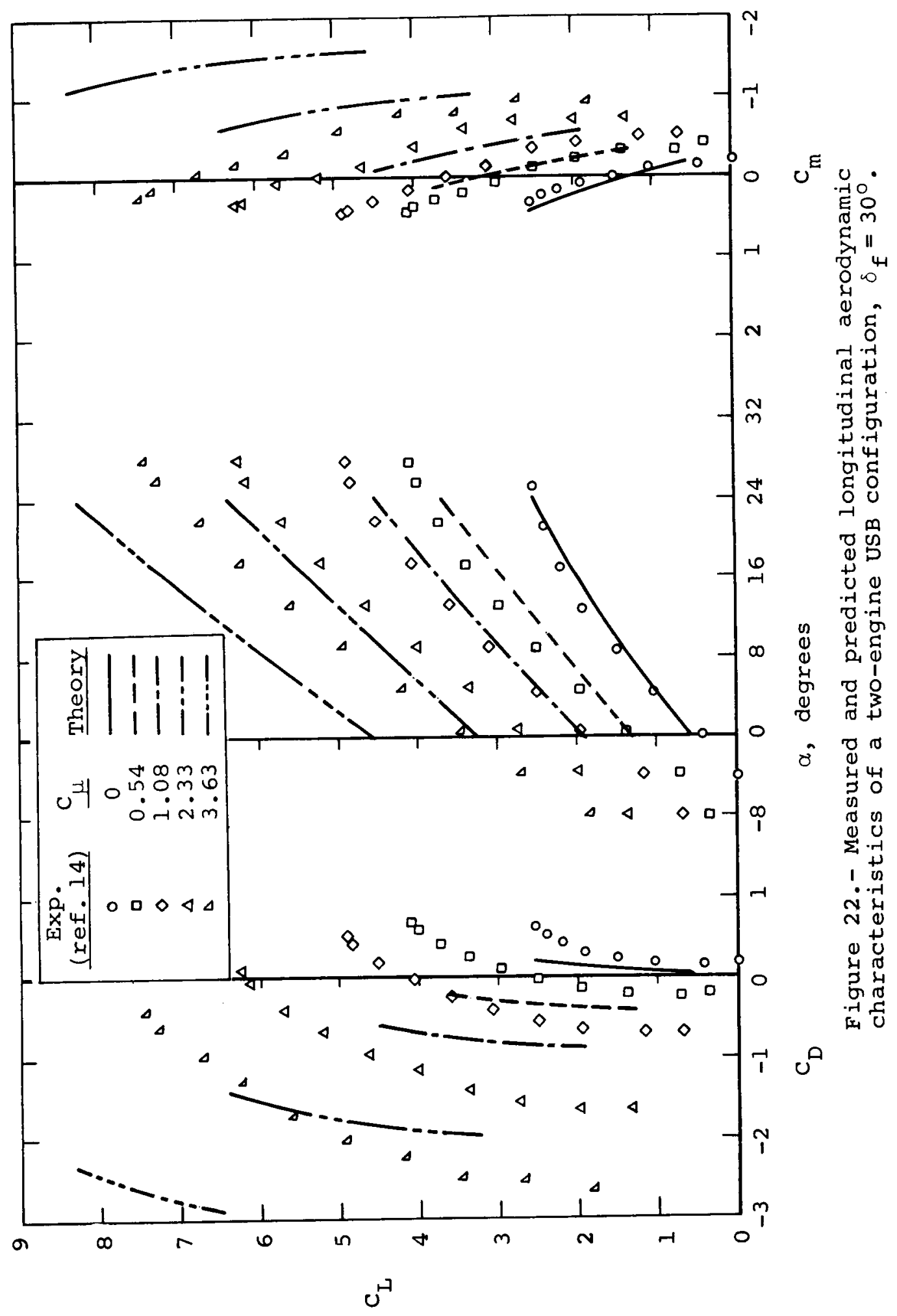




$$
\Delta C_{L}=\left.C_{L}\right|_{C_{\mu}>0}-\left.C_{L}\right|_{C_{\mu}=0}
$$

Theory

- - Experiment

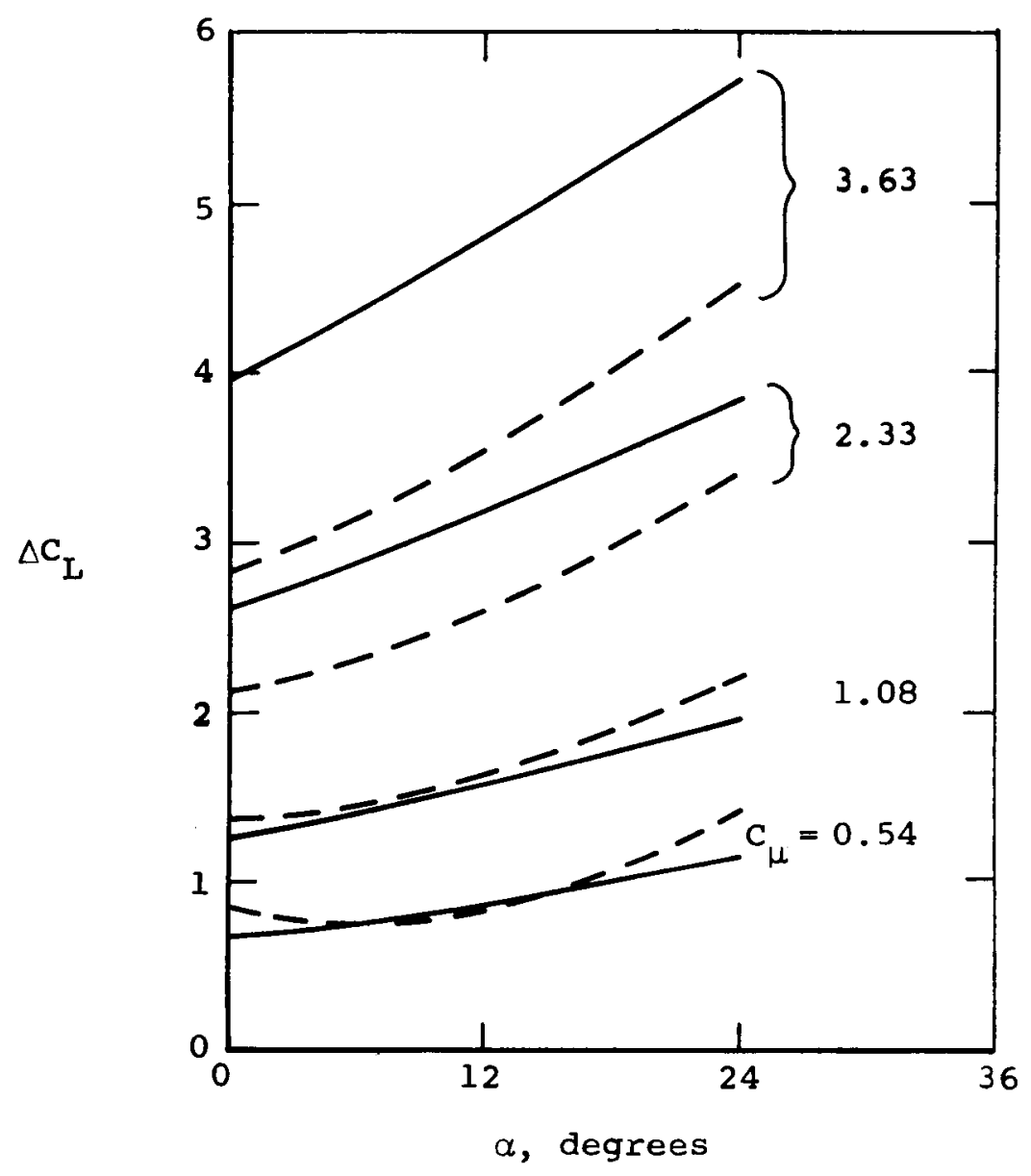

Figure 23.- Measured and predicted jet-induced lift on a two-engine USB configuration, $\delta_{f}=30^{\circ}$. 


\begin{tabular}{|c|c|}
\hline $\begin{array}{l}\text { 1. Report No. } \\
\text { NASA CR- } 3004\end{array}$ & 3. Recipient's Catalog No. \\
\hline \multirow{2}{*}{$\begin{array}{l}\text { 4. Title and Subtitle } \\
\text { CALCULATION OF THE LONGITUDINAL AERODYNAMIC } \\
\text { CHARACTERISTICS OF UPPER-SURFACE-BLOWN } \\
\text { WING-FLAP CONFIGURATIONS }\end{array}$} & $\begin{array}{l}\text { 5. Report Date } \\
\text { August } 1978\end{array}$ \\
\hline & $\begin{array}{l}\text { 6. Performing Organization Code } \\
369 / \mathrm{C}\end{array}$ \\
\hline \multirow{2}{*}{$\begin{array}{l}\text { Michael R. Mendenhall and Selden B. Spanglen } \\
\text { Mich }\end{array}$} & $\begin{array}{l}\text { 8. Performing Organization Report No. } \\
\text { NEAR TR } 157\end{array}$ \\
\hline & 10. Work Unit No. \\
\hline $\begin{array}{l}\text { 9. Performing Organization Name and Address } \\
\text { Nielsen Engineering \& Research, Inc. } \\
510 \text { Clyde Avenue } \\
\text { Mountain View, CA } 94043\end{array}$ & $\begin{array}{l}\text { 11. Contract or Grant No. } \\
\text { NAS1 }-14086\end{array}$ \\
\hline \multirow{2}{*}{$\begin{array}{l}\text { 12. Sponsoring Agency Name and Address } \\
\text { National Aeronautics \& Space Administration } \\
\text { Washington, DC } 20546\end{array}$} & $\begin{array}{l}\text { 13. Type of Report and Period Covered } \\
\text { Contractor Report }\end{array}$ \\
\hline & 14. Sponsoring Agency Code \\
\hline
\end{tabular}

Langley Technical Monitors, Robert C. Goetz and Boyd Perry III Final Report

16. Abstract

An investigation has been carried out to develop an engineering method for predicting the longitudinal aerodynamic characteristics of wing-flap configurations with upper surface blowing (USB). Two potential flow models are incorporated into the prediction method: a wing and flap lifting surface model and a jet wake model. The wing-flap model uses a vortexlattice to represent the wing and flaps. The wing may have an arbitrary planform and camber and twist, and the flap system may be made up of a Coanda flap and other flap segments of arbitrary size. The jet wake model consists of a series of closely spaced rectangular vortex rings. The wake is positioned such that it is tangent to the upper surface of the wing and flap between the exhaust nozzle and the flap trailing edge. It can be specified such that the mass, momentum, and spreading rates are similar to actual USB jet wakes.

Comparisons of measured and predicted pressure distributions, span load distributions, and total lift and pitchingmoment coefficients on swept and unswept USB configurations are included in this report. A wide range of thrust coefficients and flap deflection angles is considered at angles of attack up to the onset of stall.

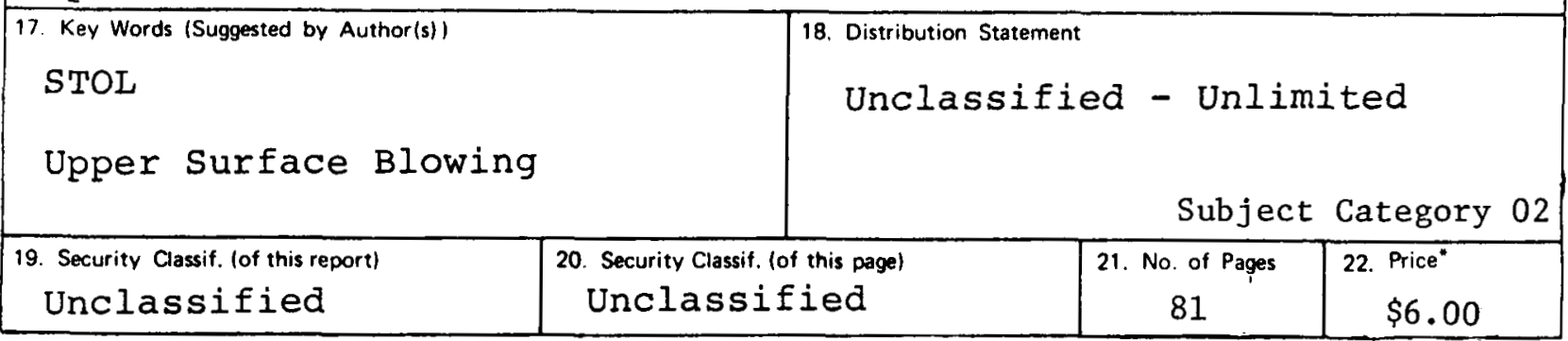

